\title{
Monitoring of hemostatic disturbances in cardio- pulmonary bypass patients : pitfalls and prospective solutions
}

Citation for published version (APA):

Bosch, Y. P. J. (2015). Monitoring of hemostatic disturbances in cardio-pulmonary bypass patients : pitfalls and prospective solutions. [Doctoral Thesis, Maastricht University]. Datawyse / Universitaire Pers Maastricht. https://doi.org/10.26481/dis.20151007yb

Document status and date:

Published: 01/01/2015

DOI:

10.26481/dis.20151007yb

Document Version:

Publisher's PDF, also known as Version of record

\section{Please check the document version of this publication:}

- A submitted manuscript is the version of the article upon submission and before peer-review. There can be important differences between the submitted version and the official published version of record.

People interested in the research are advised to contact the author for the final version of the publication, or visit the DOI to the publisher's website.

- The final author version and the galley proof are versions of the publication after peer review.

- The final published version features the final layout of the paper including the volume, issue and page numbers.

Link to publication

\footnotetext{
General rights rights.

- You may freely distribute the URL identifying the publication in the public portal. please follow below link for the End User Agreement:

www.umlib.nl/taverne-license

Take down policy

If you believe that this document breaches copyright please contact us at:

repository@maastrichtuniversity.nl

providing details and we will investigate your claim.
}

Copyright and moral rights for the publications made accessible in the public portal are retained by the authors and/or other copyright owners and it is a condition of accessing publications that users recognise and abide by the legal requirements associated with these

- Users may download and print one copy of any publication from the public portal for the purpose of private study or research.

- You may not further distribute the material or use it for any profit-making activity or commercial gain

If the publication is distributed under the terms of Article $25 \mathrm{fa}$ of the Dutch Copyright Act, indicated by the "Taverne" license above, 


\section{MONITORING OF \\ HEMOSTATIC DISTURBANCES IN CARDIOPULMONARY BYPASS PATIENTS pitfalls and prospective solutions}

Yvonne P.J. Bosch 
Cover design by Emile Bastings Webdesign

Production \& print: Datawyse / Universitaire Pers Maastricht

ISBN 9789461594679

(C) Copyright Yvonne P.J. Bosch, Maastricht 2015

Financial support by Stichting Hartsvrienden RESCAR for the publication of this thesis is gratefully acknowledged. 


\title{
MONITORING OF HEMOSTATIC DISTURBANCES IN CARDIOPULMONARY BYPASS PATIENTS pitfalls and prospective solutions
}

\author{
PROEFSCHRIFT
}

ter verkrijging van de graad van doctor aan de Universiteit Maastricht op gezag van de Rector Magnificus, Prof. Dr. L.L.G. Soete, volgens het besluit van het college van Decanen, in het openbaar te verdedigen, op woensdag 7 oktober 2015 om 16.00 uur door

Yvonne Petronella Johannes Bosch geboren op 17 januari 1979 te Roermond

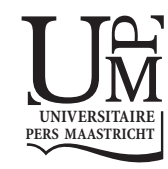




\section{Promotores}

Prof. dr. B. Mochtar

Prof. dr. J.G. Maessen

\section{Copromotores}

Dr. P.W. Weerwind

Dr. R. Al Dieri

\section{Beoordelingscommissie}

Prof. dr. P.M.H.J. Roekaerts, voorzitter

Prof. dr. N.D. Bouvy

Prof. dr. W. Buhre

Prof. dr. R.J.M. Klautz (LUMC, Leiden)

Prof. dr. M.M. Levi (AMC, Amsterdam) 
"Nobody said it was easy..."

(The Scientist, Coldplay, 2002)

Aan Joep, Pleun en Sam 



\section{CONTENTS}

$\begin{array}{ll}\text { List of abbrevations } & 8\end{array}$

$\begin{array}{lll}\text { Chapter } 1 \text { General introduction } & 9\end{array}$

Chapter 2 Aims and outline of the thesis 33

Chapter 3 An evaluation of factors affecting activated coagulation time 37

Chapter 4 Comparison of ACT point-of-care measurements: repeatability and $\quad 51$ agreement

Chapter 5 Measurement of thrombin generation intraoperatively and its association with bleeding tendency after cardiac surgery

Chapter 6 Preoperative thrombin generation is predictive for the risk of blood loss after cardiac surgery

Chapter 7 The association between rotational thromboelastometry meters and 101 blood loss after cardiac surgery with cardiopulmonary bypass

Chapter 8 General discussion

Summary

Samenvatting

Valorization

Dankwoord

Curriculum Vitae 


\section{List of abbreviations}

$\begin{array}{ll}\text { ACT } & \text { activated clotting time } \\ \text { aPTT } & \text { activated partial thromboplastin time } \\ \text { AT } & \text { antithrombin } \\ \text { AUC } & \text { area under the curve } \\ \text { AVR } & \text { aortic valve replacement } \\ \text { CABG } & \text { coronary artery bypass grafting } \\ \text { CAT } & \text { calibrated automated thrombography } \\ \text { CFT } & \text { clot formation time } \\ \text { CPB } & \text { cardiopulmonary bypass } \\ \text { CT } & \text { clotting time } \\ \text { ETP } & \text { endogenous thrombin potential } \\ \text { HSI } & \text { heparin sensitivity index } \\ \text { INR } & \text { internationalized normalized ratio } \\ \text { LMWH } & \text { low-molecular-weight heparin } \\ \text { MCF } & \text { maximum clot firmness } \\ \text { NOAC } & \text { new oral anticoagulant } \\ \text { PF4 } & \text { platelet factor } 4 \\ \text { PPP } & \text { platelet poor plasma } \\ \text { PRP } & \text { platelet rich plasma } \\ \text { PT } & \text { prothrombin time } \\ \text { ROC } & \text { receiver operating characteristic } \\ \text { ROTEM } & \text { rotational thromboelastometry } \\ \text { TEG } & \text { thromboelastography } \\ \text { TF } & \text { tissue factor } \\ \text { TFPI } & \text { tissue factor pathway inhibitor } \\ \text { TG } & \text { thrombin generation } \\ & \\ \text { TE } & \end{array}$




\title{
CHAPTER 1
}

\section{GENERAL INTRODUCTION}

\author{
Yvonne P.J. Bosch
}

Patrick W. Weerwind

Baheramsjah Mochtar

Raed Al Dieri

\section{Based on:}

Monitoring of Haemostasis and Anticoagulation in Cardiopulmonary Bypass Patients. Journal of Hematology and Thromboembolic Diseases 2014; 2:6. 


\begin{abstract}
Patients undergoing cardiac surgery with cardiopulmonary bypass are at risk of increased blood loss and transfusion requirements. Contact of blood with the surgical wound and the artificial surfaces of the extra-corporeal circuit results in a coagulopathy. Hemodilution during cardiopulmonary bypass aggravates the reduction of hemostatic factors even further. On the other hand, systemic anticoagulation using high dose unfractionated heparin challenges the adequate anticoagulant monitoring. The most frequently used test to monitor systemic anticoagulation is the whole blood activated clotting time. This has, however, proven not be a reliable test. Other hemostatic assays have their own advantages and disadvantages, but tools to monitor anticoagulation and hemostasis adequately during and after cardiopulmonary bypass are to date lacking and/or show only a weak association with clinical bleeding. Viscoelastic whole blood techniques (TEG/ROTEM) have emerged as alternatives to the routine coagulation tests, and could be more suitable for providing information about the different phases of the blood clotting. Platelet function tests give more information about the presence of platelet dysfunction, which is a major cause of bleeding after cardiac surgery. Likewise, thrombin generation tests reflect much, if not all, of the overall function of the blood clotting system. Further research of the applicability and added values of the viscoelastic whole blood tests and the thrombin generation tests in the cardiac surgical patient undergoing cardiopulmonary bypass is warranted. Simplicity in performing the test, preferably point-of-care, and rapid availability of the results, should therefore be the primary focus for targeted hemostatic interventions.
\end{abstract}

Keywords: cardiopulmonary bypass, anticoagulation monitoring, blood loss, transfusion requirements, activated clotting time, thromboelastometry, platelet function, calibrated automated thrombography 


\section{Cardiac surgery, cardiopulmonary bypass, and coagulopathy}

During cardiopulmonary bypass (CPB) for cardiac surgery, contact of blood with the surgical wound and the artificial surfaces of the extra-corporeal circuit leads to hemostatic disturbances and triggers an angry defense reaction [1, 2]. The resulting coagulopathy is caused by several factors: platelet dysfunction, low preoperative fibrinogen levels, hyperfibrinolysis, and continually produced thrombin [3-5]. In addition, hemodilution plays an important role. Patients undergoing CPB procedures are usually infused with crystalloids or colloids, used as pump prime and intra-venous fluids. These infusions lead to a state of dilution coagulopathy while there is a significant fall in clotting factors [3]. Secondly, excessive dilution could lead to an overestimation of the heparin effect, and a resulting overestimation of the required protamine neutralizing dose [6]. All these factors are predictive for an increased risk of excessive perioperative blood loss and subsequent transfusion requirements $[7,8]$. Other clinical consequences of coagulopathy are: mediastinal re-exploration, postoperative stroke due to hypercoagulability and increased mortality [2,9]. Furthermore, intensive care stay is prolonged and hospital costs are increased [7]. Hemostatic activation seems to be unavoidable in patients undergoing $\mathrm{CPB}$, thereby upsetting their postoperative recovery.

\section{Heparin anticoagulation}

Cardiac surgical patients undergoing CPB are typically anticoagulated using a very high dose of unfractionated heparin. This is the only anticoagulant that brings about an immediate effect and, that can be monitored bedside by a point-of-care test. Moreover, unfractionated heparin is easily neutralized by protamine at the end of the procedure [10]. The primary anticoagulant action of heparin appears to result from its binding with antithrombin (AT). The predominant action of AT is inactivation of factor Ila (thrombin) and factor Xa [11]. Following activation of AT, heparin is released and becomes available for interaction with other AT molecules. In contrast, AT is consumed and AT levels are progressively depleted [11]. Therefore, decreased heparin responsiveness is often attributed to AT deficiency. Besides binding with AT, heparin may bind thrombin directly or act through heparin-cofactor II. Heparin also exerts an anticoagulant effect by augmenting the activity of tissue factor pathway inhibitor (TFPI) [11]. Measuring the real anticoagulant effect of heparin is complicated and thus depends on many variables which determine the patients sensitivity to heparin, such as: 1) the availability of AT; 2) the binding of heparin to proteins (such as albumin and glycosamineglycans) in the bloodstream [12]; 3) the binding of heparin to endothelium 
[11]; and 4) platelets: both the release of platelet factor 4 and platelets themselves are heparin neutralizing [13].

\section{Monitoring of anticoagulation in cardiac surgery}

Several laboratory methods are currently available for monitoring hemostasis and anticoagulation during cardiac surgery. Table 1 shows an overview of available hemostatic tests. Advantages and disadvantages are also listed.

\section{Determination of heparin concentration}

Heparinization is currently monitored by either measuring heparin concentration or by measuring its effect on clotting times. Chromogenic tests for measuring heparin concentration are less sensitive to interfering substances than the clotting tests are. Plasma heparin concentration is determined via its accelerating function in the inhibition of factor Xa or thrombin by AT. Although thrombin based tests are possible, the majority of the commercial heparin assays are performed using anti-factor Xa-activity (anti$\mathrm{Xa})$, in the hematology laboratory. There are two different designs of the anti-Xa assay. Some tests use a saturating concentration of AT in the reagent. This test determines the 'total heparin'. The other design does not add AT and relies on the AT in the sample. This determines the AT/heparin complex (or the 'active heparin') [14]. Heparin concentration can also be measured bedside by a hemostasis management system (Hepcon HMS, Medtronic, Minneapolis MN). This is a multichannel clot-timing instrument that uses the principle of heparin-protamine titration to quantitatively determine the heparin concentration in whole blood. In each channel, a plastic rod is rapidly lifted and dropped by an oscillating metal bar. Whole blood is automatically placed into each channel by a $3 \mathrm{ml}$ syringe inserted into the machine. The time for blood to clot is displayed for each channel [15]. Six levels of heparin can be determined by using discrete cut offs [16]. Excess protamine inhibits clot formation and insufficient protamine does not fully antagonize heparin, therefore the channel with the fastest clot formation will represent the protamine concentration that optimally neutralizes the existing heparin [17]. The imprecision of the Hepcon HMS could potentially be caused by the effects of release of TFPI by heparin in vivo but not ex vivo. In the absence of an endothelial source of TFPI, the contribution of TFPI to heparin responsiveness may be underestimated by the Hepcon HMS [16]. Heparin concentration monitoring has not uniformly been associated with less bleeding, as it may be associated with heparin rebound postoperatively [17]. Between the anti-Xa assay and the HMS assay there is a lack of agreement [18]. It can be postulated that the HMS assay is affected by factors like 
hemodilution, hypothermia and platelet dysfunction [18], while the anti-Xa assay only reflects the heparin concentration that inhibits factor Xa. The fraction of heparin that is responsible for anti-factor Ila activity is not measured, meaning that the anti-Xa assay will not give a real and complete reflection of heparin concentration [19]. Thus, monitoring of heparin concentration is only a surrogate for monitoring of anticoagulation, since there are important variations in the response of patients to heparin [20]. 


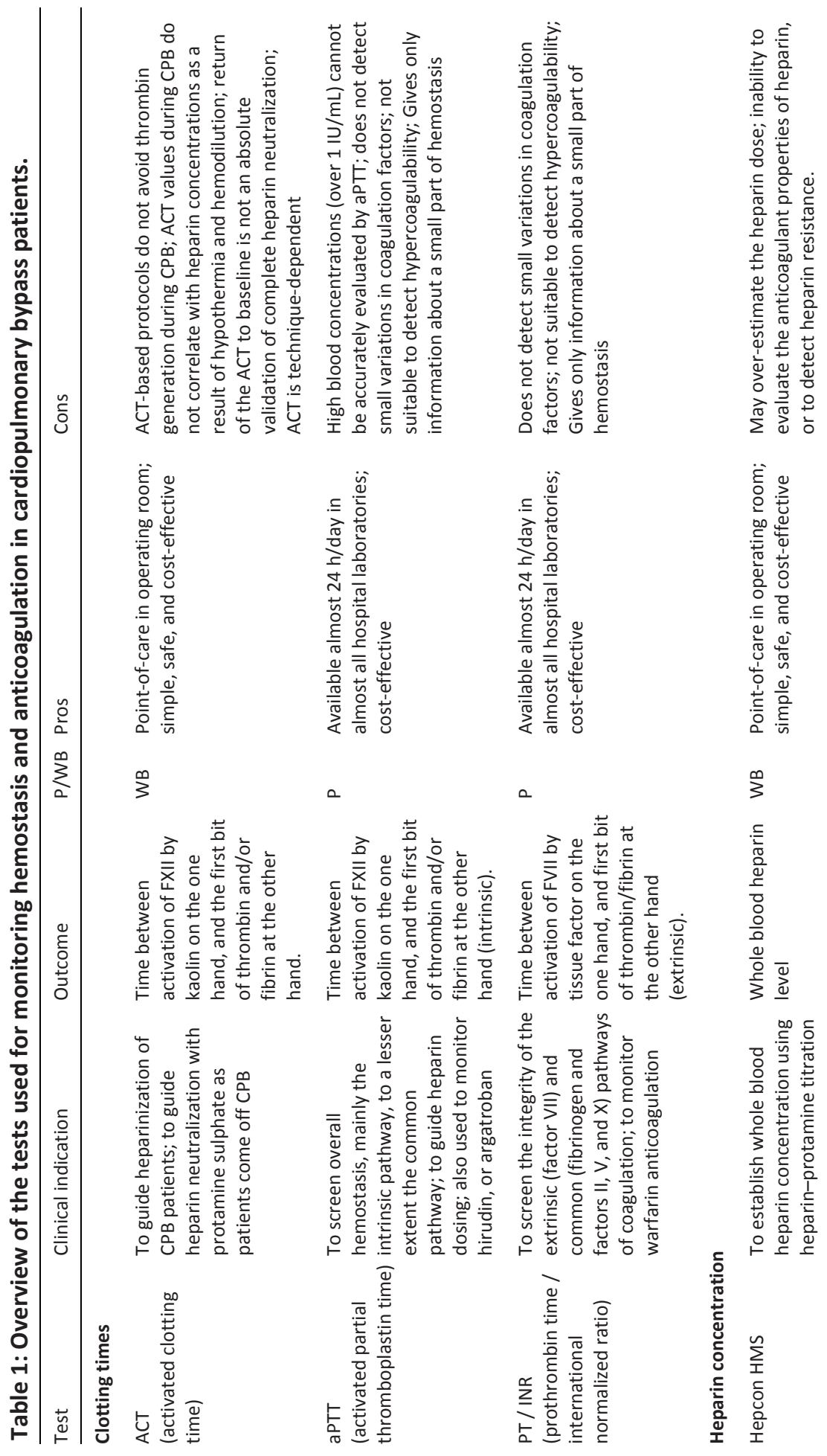




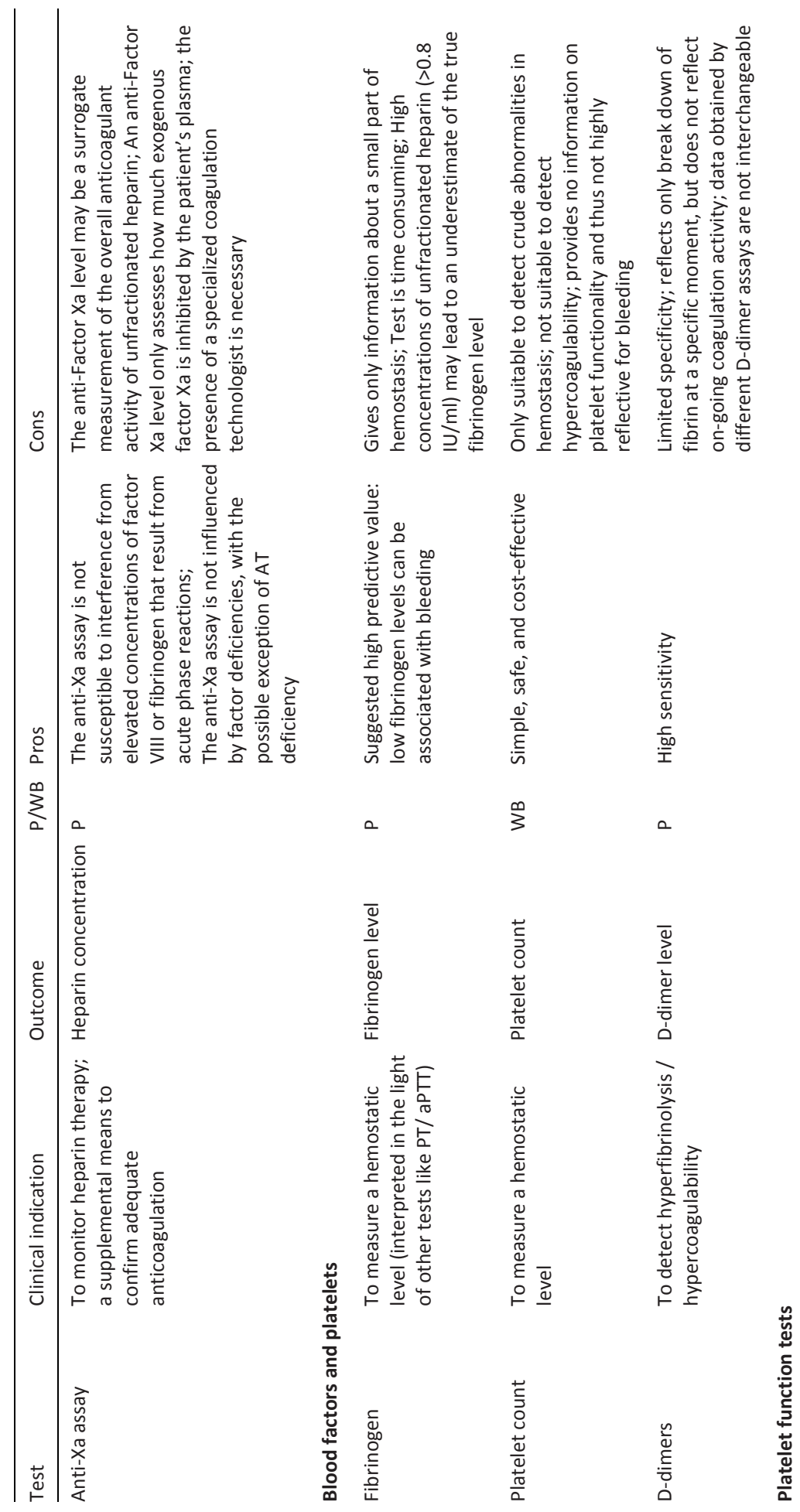




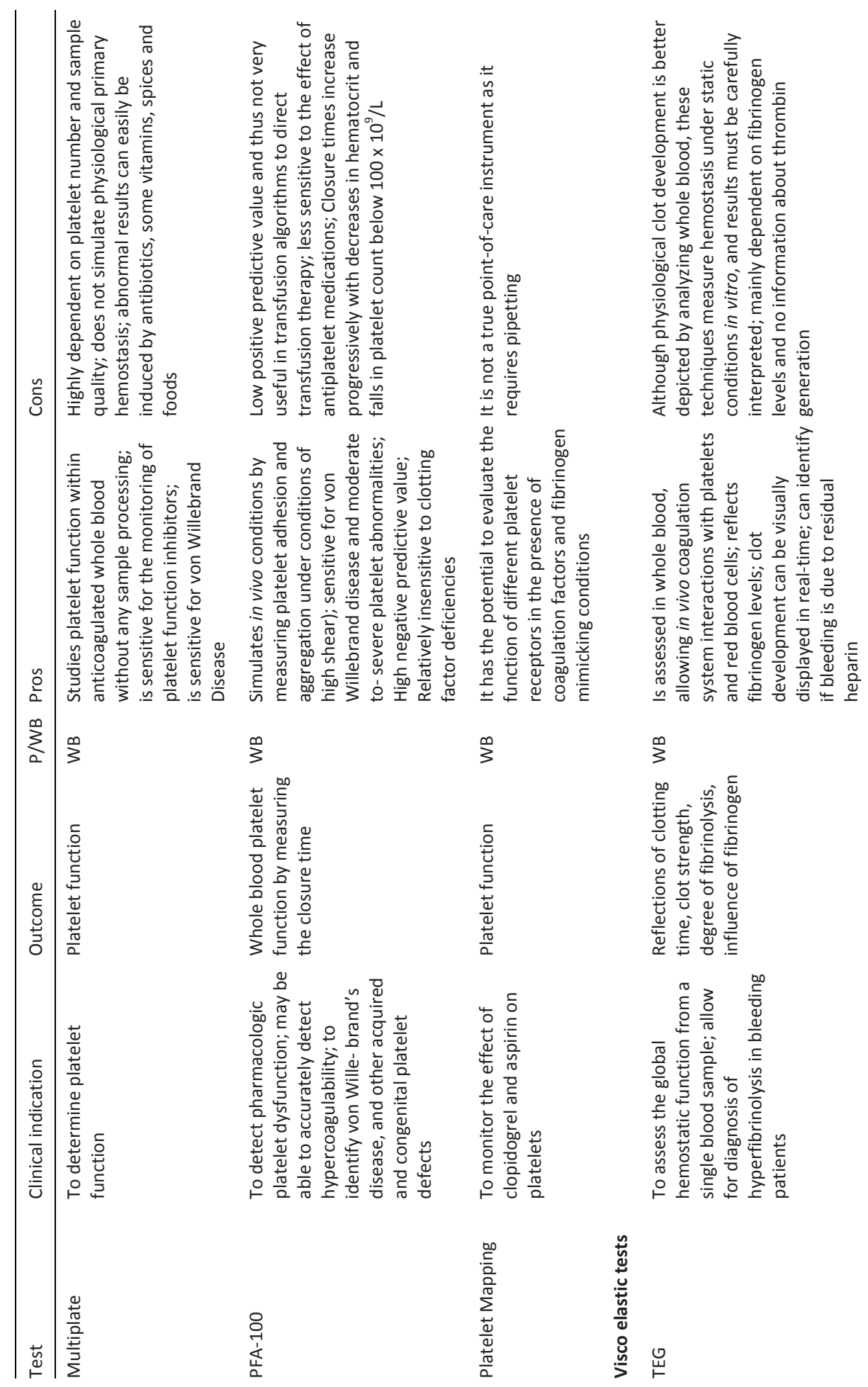




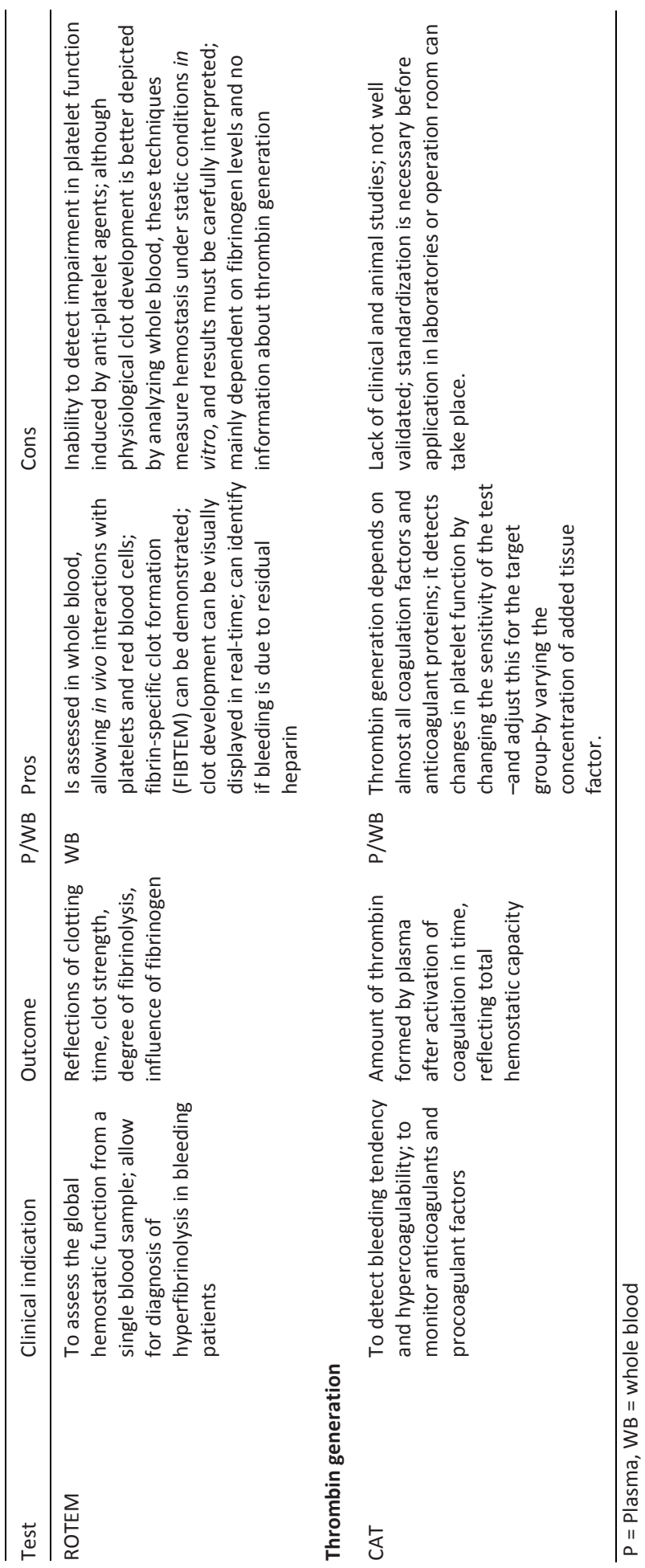




\section{Clotting-time based methods}

Although routine preoperative coagulation testing contributes little to patient care, this is justified in case of cardiac operations with CPB since patients will undergo an operation with major effects on hemostasis and many will require blood transfusions [21]. Prothrombin time (PT) and activated partial thromboplastin time (aPTT), performed in plasma, are by far the most common screening tests for coagulation abnormalities. These tests correspond respectively to the extrinsic and the intrinsic pathways of coagulation. PT monitoring is widely used as a preoperative screening test for patients receiving oral anticoagulation. The sensitivity for prothrombin and fibrinogen is less pronounced than for other factors. The international normalized ratio (INR) is the ratio of the PT of the patient and the normal PT standardized with the international sensitivity index (ISI). This is to correct for specific laboratory methods. The aPTT is used to guide unfractionated heparin $(<1 \mathrm{IU} / \mathrm{ml})$ clinically. After heparin neutralizing, the aPTT could be measured to control heparin rebound. The PT and APTT do not reflect the complexity of hemostasis in vivo. One disadvantage of the PT and aPTT is the impossibility to provide information about the interaction with platelets since it is measured in platelet poor plasma. Consequently, these tests will not reflect the extent of bleeding, as platelet count and function may vary [22]. It was clear that even though deficiencies of each of the factors in the intrinsic pathway could have equally long aPTT values, they had dramatically different risks of hemorrhage [23]. Hence the PT and aPTT, providing information on the ability to clot, are poor predictors of bleeding despite their widespread use [24-27]. Another disadvantage is that the PT and aPTT remain normal under hyperfibrinolytic conditions, meaning that bleeding caused by increased fibrin breakdown will not be detected [22]. Although there are many available coagulation assays, it is rather complicated to find the ideal test reflecting the clinical situation of the CPB patient.

During cardiac surgery the prolongation of the clotting time as a reflection of the anticoagulant status is measured. This is performed by the point-of-care activated clotting time (ACT). The principle of the ACT is adding whole blood to a tube containing a contact activator, such as kaolin, celite or silica. Clotting activation and fibrin formation will be initiated and detected. The ACT was first described by Hattersley in 1966 [28], and introduced into cardiac surgery by Bull in the 70's [29]. The safe minimal level of the ACT was first proposed at $300 \mathrm{~s}$. However, this was stepwise increased to $480 \mathrm{~s}$, after observing visible clots in the extracorporeal circuit and because of a three to six fold variation in heparin effect and a fourfold variation in heparin-life. The most important cofactor that heparin requires for its anticoagulant effect is AT. Therefore, it is surprising that there is no relation between the ACT and AT level [30-32]. In 
the early 90's variability between the different systems was noted, i.e. ACT analyzers differ by activators, detection systems, and use different sample volumes [33-35]. Consequently, the ACT value differs for each analyzer, suggesting that extrapolation between different systems would not be appropriate [36]. The ACT is also influenced by factors like the balance of coagulation factors and inhibitors, fibrinogen, platelets, plasma proteins which bind heparin, TFPI release from vasculature, increased hemodilution, and hypothermia of the patient. Nowadays, growing evidence suggests that coagulation is increasingly activated with time during CPB, even with ACT-based protocols [37]. This might lead to a progressive consumptive coagulopathy resulting in a prolonged ACT although heparin levels are decreased, resulting in an incorrect estimation of the anticoagulation status [38]. As a result, thrombin might be only partially suppressed and microscopic emboli are produced [2]. Further, the majority of thrombin generation is triggered via factor VIla and tissue factor, whereas the ACT reflects inhibition of contact activation, which is not indicative for the amount and time of thrombin generation [39]. Additionally, it is questionable whether adequate heparin neutralization will be achieved postoperatively, since ACT is poorly sensitive to low heparin concentrations. This could result in over dosage of protamine, or incomplete heparin reversal [33]. Measuring the anticoagulant effect of heparin with the ACT, may have little or no relationship to what is clinically happening [11]. Therefore, when monitoring anticoagulation exclusively with the $\mathrm{ACT}$, there are a lot of pitfalls to overcome.

\section{Monitoring of hemostasis in cardiac surgery}

The complex process of hemostasis can be broken down into four components: platelet activation, thrombin generation, clot formation/stabilization, and fibrinolysis [40]. To get a better understanding of these processes during and after CPB, it would be desirable to have a coagulation test that provides information about all these phases. However, such a tool to monitor hemostasis adequately is lacking [2, 8, 41, 42]. Standard coagulation tests do not reflect the activity of both the coagulation factors and the natural anticoagulant proteins, and the interaction with platelets [8, 22]. In addition, tests are often time consuming and the results not rapidly available, so they do not reflect the current state of the coagulation system and could lead to inappropriate treatment [43]. At present a combination of hemostatic assays may provide more effective diagnostic information than the use of any single test [44], because the current tests are on its own often limited in utility. 


\section{Standard hemostatic assays}

Platelet count is only a quantitative measurement and, does not give any information about the platelet functionality, therefore it is less reflective for bleeding [45]. However, in studies by Karkouti et al. [46] and Coakley et al. [47] it was shown that loss of platelets reflected by both pre- and post- CPB platelet count was associated with increased blood loss. Fibrinogen on the other hand is only a single factor, excluding the influence of other blood cells. Large decreases in fibrinogen and subsequent low fibrinogen levels both pre- and post- $\mathrm{CPB}$, are shown to be predictive for increasing blood loss $[46,48]$. Though, Coakley et al. did not find postoperative measured fibrinogen to be associated with blood loss. Similarly, low plasma concentrations of fibrinogen were not significantly associated with increased homologous blood usage [49]. $D$ dimer levels are split products of Factor-XIII cross-linked fibrin and are determined with monoclonal antibody assays. An increase of D-dimer over the baseline value indicates excessive fibrin formation, stabilization by Factor-XIIla and subsequent fibrinolysis [50], that is measured at a specific moment, but which does not reflect on-going coagulation activity. D-dimer levels do increase during cardiac surgery, but there is no association with blood loss [51]. Kuepper et al. concluded that the D-dimer assay has a relatively high negative predictive value (measured 15 minutes after protamine administration), which might be useful to identify patients who are unlikely to have an excessive postoperative bleeding [50]. This could be helpful to avoid unnecessary transfusions after cardiac surgery. One should be critical to interpret the results of D-dimer assays, because there is a known variability among different assays due to heterogeneity of fibrin degradation products in patient samples, reactivity of antibodies, different calibrators and different assay formats [50].

\section{Viscoelastic whole blood techniques}

Viscoelastic whole blood techniques seem to be more suitable for reflecting the complex coagulation system. Thromboelastography (TEG) was first described by Hartert in 1948 [52]. Using this assay, whole blood is added to a cup in which a pin is suspended connected to a detector system. The cup initiates the movement and the viscoelastic changes that occur during coagulation are measured and recorded in a graphical representation of the fibrin polymerization process. This technique was very sensitive to vibrations with the resultant unstable reproducibility. Over the years, the technique was refined, and nowadays there are two systems available: the TEG (Haemoscope Corporation, IL, USA) using the original principle, and the rotational thromboelastometry (ROTEM, TEM International GmbH, Munich, Germany), which uses a modified principle where the pin inside a standing cuvette is moving. The TEG device has two inde- 
pendent measuring channels, which includes a platelet mapping assay. The latter provides information about a patients' response to anti-platelet therapy. ROTEM is also performed in whole blood, and it provides therefore information on the contribution of fibrinogen and platelets to clot formation. The rate of fibrin polymerization as well as the overall clot strength is assessed. During the clotting process (conversion of fibrinogen to fibrin), the clot increasingly restricts the rotation of the pin with rising clot firmness. This is detected and converted in a graph of clot formation and subsequent lysis. ROTEM is suitable for a more timely assessment of fibrinogen levels $(<15-20$ minutes). The following different measurements can be performed in four independent channels:

EXTEM extrinsically activated coagulation, by tissue factor

INTEM intrinsically activated coagulation, by ellagic acid

FIBTEM extrinsically activated coagulation, by tissue factor; any contribution of platelets to clot formation is eliminated by cytochalasin D, allowing detection of fibrinogen deficiency or fibrin polymerization disorders.

APTEM extrinsically activated coagulation, by tissue factor; aprotinin is added in order to block fibrinolysis; this reveals hyperfibrinolysis when compared with the EXTEM.

HEPTEM intrinsically activated coagulation, by ellagic acid, contains heparinase to eliminate any heparin effect. Compared with the INTEM assay, it specifically confirms the presence of residual heparin.

Table 2: Nomenclature used for TEG and ROTEM.

\begin{tabular}{lll}
\hline Definition & TEG & ROTEM \\
\hline $\begin{array}{l}\text { Time between addition of the activator and start of clot } \\
\text { formation }\end{array}$ & r value & coagulation time (CT) \\
$\begin{array}{l}\text { Time between start of clot formation and reaching an } \\
\text { amplitude of } 20 \mathrm{~mm}\end{array}$ & k value & clot formation time \\
Steepness of curve & alpha angle & alpha angle \\
$\begin{array}{l}\text { Greatest amplitude } \\
\text { Amplitude measured } 60 \text { minutes after onset of clotting, as } \\
\text { a percentage of the greatest amplitude }\end{array}$ & lysis index & maximum clot firmness \\
\hline
\end{tabular}

The nomenclature used for the TEG and the ROTEM are presented in Table 2, and the graphical representation of the ROTEM is shown in Figure 1. The curve of the TEG is comparable with the curve of the ROTEM.

A disturbed activation of coagulation is indicated by a prolonged clotting time. An abnormal clot formation is indicated by a prolonged clot formation time and/or re- 
duced clot firmness. Fibrinolysis is detected by lysis of the clot (>15\%) or, in case of ROTEM, by finding of a better clot formation (shorter CFT, greater MCF) in APTEM as compared to EXTEM. The clot formation time is used as a guide for fresh frozen plas$\mathrm{ma}$, the clot strength to judge platelet infusion. The degree of lysis is used to indicate the need for antifibrinolytic therapy.

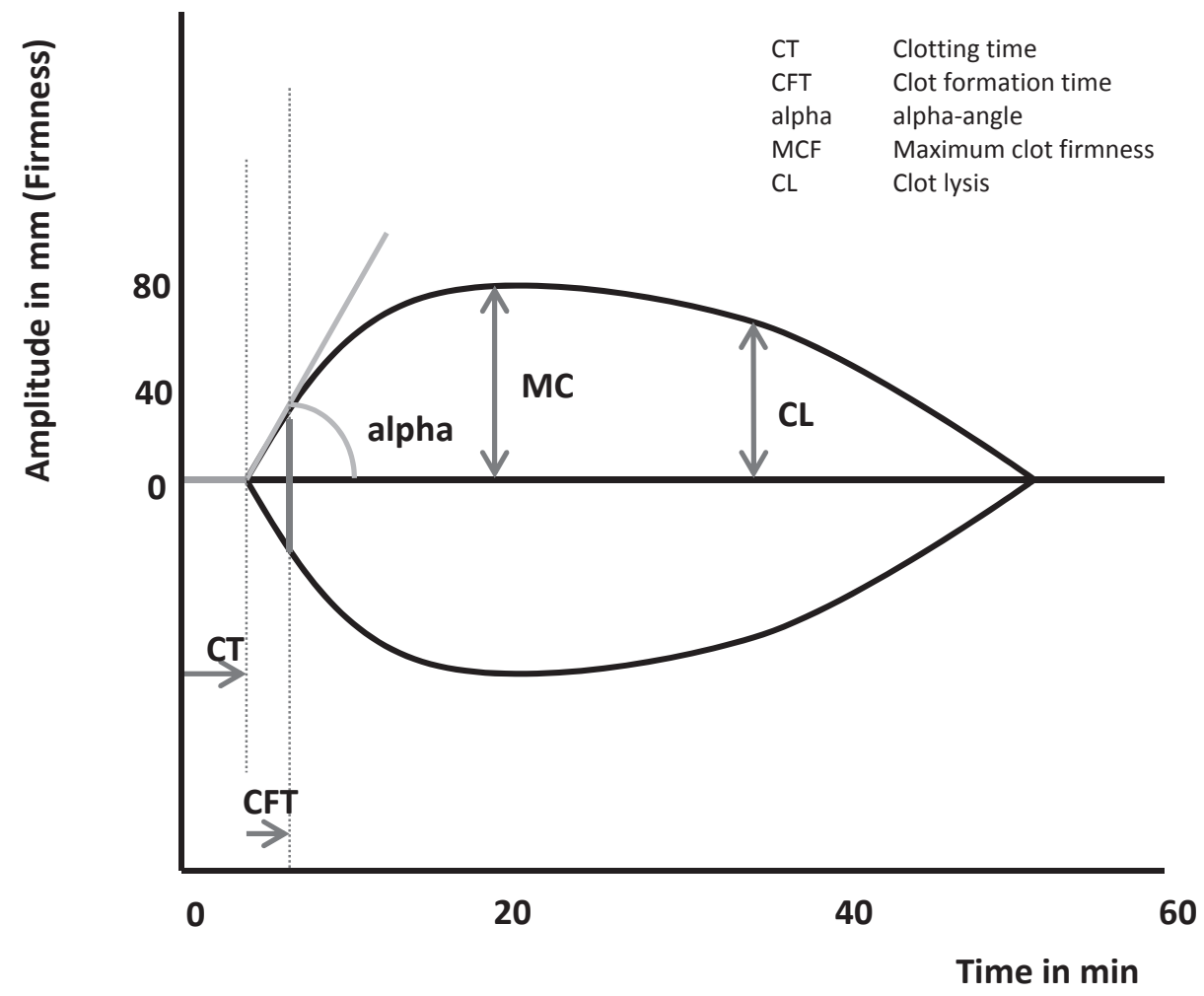

Figure 1: Schematic presentation of ROTEM tracing: The commonly reported variables are indicated: clotting time (CT), clot formation time (CFT), alpha angle $(\alpha)$, maximum clot firmness (MCF) and clot lysis (CL).

Besides the primary difference of the movement of the cup and the pin, a second difference between the TEG and the ROTEM assay is the detector system, which is a torsion wire in the TEG, and an optical detector in the ROTEM [53]. Thirdly, the TEG system uses a kaolin/phospholipid mixture as activator, whereas in the ROTEM either ellagic acid or tissue factor is used. Lastly, the TEG uses a larger and less diluted blood volume in the cuvette [54]. Results in viscoelastic whole blood measurement using either TEG or ROTEM are therefore not completely interchangeable and clinical interpretation should be used with caution [54]. 
Wasowicz et al. [55] concluded in an observational study comprising 434 CPB patients that on-CPB thromboelastography could improve risk stratification for excessive blood loss postoperatively. In contrast, Coakley et al concluded that thromboelastometry was not predictive for postoperative bleeding in cardiac surgical patients undergoing CPB [47]. Consumption of allogenic blood products tends to decrease in case viscoelastic tests are used [56-58]. However, viscoelastic clot-based assays are mainly dependent on fibrinogen levels $[47,59,60]$. A great deal of hemostasis is not captured by the fibrin clotting endpoints used [61]. Only $5 \%$ of thrombin is formed when clotting-based assays terminated. This is not directly related to hemostasis and thrombosis, like thrombin concentrations are, but a covariable of one of the effects of thrombin formation, i.e. fibrin (-monomer) polymerization [62].

\section{Platelet function tests}

Although thrombocytopenia occurs during cardiac surgery, this alone infrequently leads to increased blood loss if the platelet function is normal [17]. Platelet dysfunction, is one of the most important causes of non-surgical bleeding after CPB, and is not assessed by standard hematology laboratory assays [15]. Nowadays, several platelet function tests are available. We will discuss a test based on aggregation, one based on shear stress, and the last based on platelet contribution to clot strength.

Classical light transmission aggregometry performed in platelet-rich plasma is the "gold standard" for platelet function testing $[63,64]$. The Multiplate analyzer is an instrument with five channels for parallel aggregometry measurements in whole blood. Blood and reagents were pipetted into test cells containing four electrodes that formed two independent sensor units. The ability of platelets to adhere to the metal sensors was measured by the change of electrical resistance between two sensor wires. This impedance change is plotted against time and the area under the aggregation curve was used to measure the aggregation response, quantified in arbitrary aggregation units $(U)$. Whole blood platelet aggregometry is likely affected by a reduced hematocrit and becomes unreliable at platelet counts $<50 * 10^{9} / /[63,65]$. The Multiplate device will produce a result in 10 minutes after sampling, except when adenosine diphosphate is used as an agonist [63]. Unfortunately, it does not reflect the complex interaction between thrombin, fibrinogen and platelets, and the end result of the coagulation process, the formation of the clot. Data showing correlation with blood loss are lacking [64].

The PFA-100 platelet function analyzer is an in vitro system that provides a measure of platelet function in citrated whole blood. The PFA-100 assesses the time taken for a platelet plug to occlude a microscopic aperture within a membrane coated with platelet agonists, collagen and, depending on the cartridge, epinephrine or adenosine 
diphosphate. The time from the start of the test until the occlusion of the aperture by a platelet plug was reported as the PFA-100 closure time (s). The test can be performed in 5-8 minutes. The PFA-100 system provides automated assessment for inherited, acquired (CPB), or drug-induced platelet dysfunction.

The Platelet-Mapping assay is a modification of the TEG, measuring percentage platelet aggregation in the presence of ADP or arachidonic acid. It was designed to monitor the effect of clopidogrel and aspirin on platelets and in decision-making for timing of cardiac surgery or other invasive procedures after discontinuation of antiplatelet therapy. This assay uses a viscoelastic test in whole blood to measure the clot strength (maximum amplitude) and detects the percentage of platelets inhibited by aspirin and clopidogrel. TEG platelet-mapping tracing shows the strength of coagulation formed by activated platelets [66].

In general, platelet function analyzers are sensitive enough to detect the effects of antiplatelet drugs on platelet function in cardiac surgical patients [15, 63, 64]. There is evidence that platelet function analyzers were able to predict patients at risk for increased postoperative blood loss [66-69] when performed in either the pre- or post$\mathrm{CPB}$ setting. However, after correcting for hematocrit and platelet count there were no differences anymore between bleeders and non-bleeders measured by the PFA-100 [69], what led to the conclusion that PFA-100 is not useful for routine use after cardiac surgery [69]. Rahe-Meyer et al. found no correlation between decreased perioperative platelet aggregometry measured by Multiplate and blood loss in the 24 hour period after surgery [65]. Although both low pre- and post-operative platelet function tests defined groups of patients with significantly higher transfusion of platelet concentrates [65-66], there is no consensus to conclude that measuring platelet function is useful in transfusion algorithms [15, 37].

\section{Calibrated Automated Thrombography}

Thrombin is the most important enzyme in the thrombotic portion of the hemostatic reaction during CPB. Therefore, monitoring thrombin generation (TG) is of utmost importance. TG reflects much - if not all - of the overall function of the blood clotting system [62] and plays several roles such as modulating the cleavage of fibrinogen to fibrin, activating platelets and the process of fibrinolysis [61, 70]. It extends from coagulation activator and inhibitor to cellular regulator. In 1953 the thrombin generation test was described by Macfarlane and Biggs [71], as to be a valid function test of the clotting system. Later, the calibrated automated thrombography (CAT) test, following triggering of plasma with tissue factor, was developed by Hemker and co-workers [62, 72,73 ] for monitoring of the rate and extent of TG by the continuous measurement of the fluorescent split-product of the fluorogenic substrate. The course of TG is graph- 
ically represented in a thrombogram. TG is measured in a fluorometer equipped with 96 wells. In one well a calibrator has been added. In a second well TG is triggered with tissue factor and phospholipids in platelet poor plasma (PPP), and only with tissue factor in platelet rich plasma (PRP). A fresh mixture of fluorogenic substrate (Z-Gly-GlyArg-AMC) and $\mathrm{CaCl}_{2}$ (called FluCa) is dispensed to all the wells to be measured. The readings from the TG are compared to the readings of the calibrator well, thrombin concentration is calculated and thrombin concentration in time is displayed. CAT parameters are: 1 ) lag time $(\min )$ : the initiation phase of clotting, that equals the clotting time; 2 ) peak height (nM): the maximal amount of thrombin formed; 3 ) endogenous thrombin potential (ETP) ( $\left.\mathrm{nM}^{*} \mathrm{~min}\right)$ : the area under the TG curve, representing thrombin generation and decay in time; and 4) time to peak (min): the time needed to achieve the peak height (Figure 2). The total time course of CAT to provide the required clinical relevant information could be less than 30 minutes (depending on the hemostatic condition of the patient). The first parameters are already known within 5 minutes.

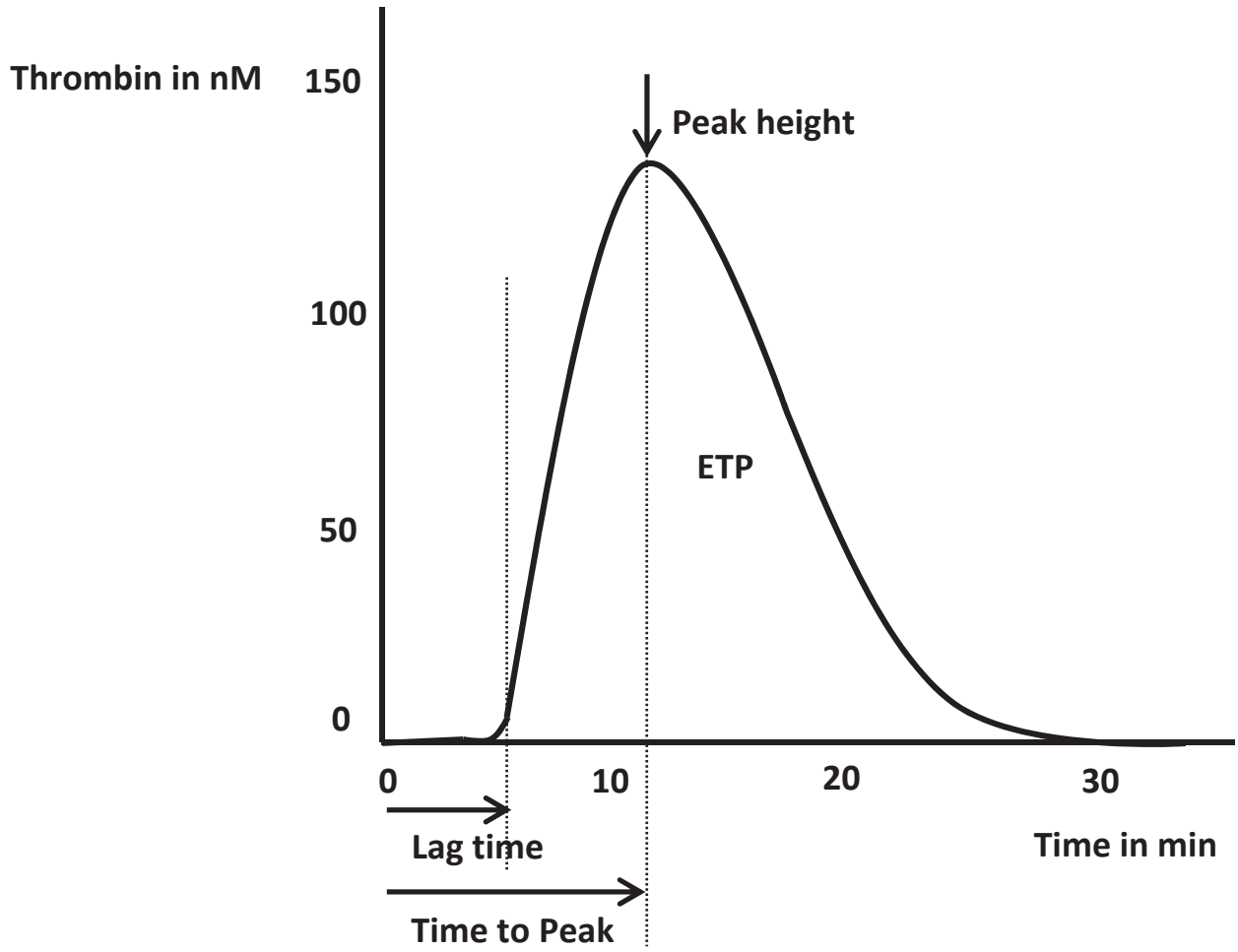

Figure 2: Schematic presentation of thrombin generation test:

The commonly reported variables are indicated: lag time, peak height, time to peak and endogenous thrombin potential (ETP). 
Recently, it became possible to measure TG in whole blood instead of plasma. This is an important development as it is approaching the in vivo conditions more closely. No centrifugation step is required, which could lead to uncontrolled variations in cell counts and activation of blood cells. The whole blood CAT assay is described elsewhere [74].

CAT can be used for detection and quantification of bleeding tendency, for detecting hyper coagulability, and assessing substitution and antithrombotic treatments. There is already evidence that thrombin generation measured by CAT is predictive for increased bleeding risk after cardiac surgery [47, 75-77], however, further research involving the clinical benefits is warranted.

\section{Optimal approach for monitoring of hemostasis in cardiac surgical patients undergoing CPB}

The ideal way to monitor hemostasis and anticoagulation during CPB, should be a test that includes all factors that interact to create hemostasis at a specific moment: blood cells, plasma proteins, contributions of the vessel wall, flow conditions, and the influence of anticoagulants. Including all these aspects into one assay will reflect closer the in vivo circumstances. Measuring thrombin generation in whole blood could be such a test. Besides the most ideal test conditions, the test should also be clinically useful. The clinical utility of coagulation tests is the likelihood that a test result will lead to an improved health outcome. In cardiac surgery this means to get more control over individual blood loss and to facilitate optimal, targeted administration of pharmacological agents and blood components in case of excessive bleeding [78]. The clinical consequences of a disturbed hemostatic condition could be reduced with more accurate control of heparin anticoagulation and its reversal. Novel pharmacological approaches have resulted in the introduction of new oral anticoagulants (NOACs) into the adult clinical setting. These direct thrombin and factor Xa inhibitors have been developed to reduce the well-established drawbacks of currently available anticoagulants (vitamin $\mathrm{K}$ antagonists and heparins). They are currently administered at fixed doses without control of their effect on the clotting system. However, measurement of their anticoagulant effect may be desirable in situations like bleeding and emergent surgery particularly when patients did not quit intake of NOACs. Whether NOACs require routine monitoring cannot be answered at present, as relevant data are lacking and monitoring is inadequate. Another practical aspect of running the ideal test is the simplicity, and results should be rapidly available. Point-of-care, preferably at the patient's bedside, would be the goal of hemostatic monitoring, where interventions could be done immediately. Obviously, development of coagulation monitors in cardiac surgery starts 
with understanding the process of coagulation, the disturbances of coagulation, but also the snags and pitfalls. The clinical utility, especially prediction of bleeding postoperatively, is the next step in investigating available tests. The most ideal goal is to create an optimal test from bench to bedside in the near future.

\section{Conclusion}

Earlier diagnosis of hemostatic abnormalities and risk of bleeding in cardiac surgical patients undergoing cardiopulmonary bypass is warranted. Viscoelastic whole blood tests could improve timely identification of hemostatic disturbances. However, CAT gives a more complete reflection of the overall blood coagulation system. Further research of the clinical applicability of viscoelastic tests and CAT, preferable in whole blood, is required. Finally, several improvements such as simplicity in running the test, and a short turnaround time would be highly desirable. 


\section{References}

1. Despotis GJ, Gravlee G, Filos K, Levy J (1999) Anticoagulation monitoring during cardiac surgery: a review of current and emerging techniques. Anesthesiology 91: 1122-1151.

2. Edmunds LH Jr, Colman RW (2006) Thrombin during cardiopulmonary bypass. Ann Thorac Surg 82: 2315-2322.

3. Chandler WL (2005) Effects of hemodilution, blood loss, and consumption on hemostatic factor levels during cardiopulmonary bypass. J Cardiothorac Vasc Anesth 19: 459-467.

4. Walden K, Jeppsson A, Nasic S, Backlund E, Karlsson M (2014) Low preoperative fibrinogen plasma concentration is associated with excessive bleeding after cardiac operations. Ann Thorac Surg 97(4): 1199-1206.

5. Woodman RC, Harker (1990) LA, Bleeding complications associated with cardiopulmonary bypass. Blood 76: 1680-16

6. Bull BS, Hay KL, Herrmann PC (2009) Postoperative bypass bleeding: a bypass-associated dilutional (BAD) coagulopathy? Blood Cells Mol Dis 43: 256-259.

7. Gorlinger K, Shore-Lesserson L, Dirkmann D, Hanke AA, Rahe-Meyer N, et al. (2013) Management of hemorrhage in cardiothoracic surgery. J Cardiothorac Vasc Anesth 27: S20-34.

8. Innerhofer P, Kienast J (2010) Principles of perioperative coagulopathy. Best Pract Res Clin Anaesthesiol 24: 1-14.

9. Bevan DH (1999) Cardiac bypass haemostasis: putting blood through the mill. Br J Haematol 104: 208219.

10. Koster A, Fischer T, Gruendel M, Mappes A, Kuebler WM, et al. (2003) Management of heparin resistance during cardiopulmonary bypass: the effect of five different anticoagulation strategies on hemostatic activation. J Cardiothorac Vasc Anesth 17: 171-175.

11. Blaisdell, FW (1996) Matas Lecture. Heparin-controversies and misconceptions. Cardiovasc Surg 4: 691700.

12. Hirsh J, Raschke R, Warkentin TE, Dalen JE, Deykin D, et al. (1995) Heparin: mechanism of action, pharmacokinetics, dosing considerations, monitoring, efficacy, and safety. Chest 108: 258S-275S.

13. Horne MK, Chao ES (1989) Heparin binding to resting and activated platelets. Blood, 74: 238-243.

14. Walenga JM, Hoppensteadt DA (2004) Monitoring the new antithrombotic drugs. Semin Thromb Hemost 30: 683-695.

15. Avidan MS, Alcock EL, Da Fonseca J, Ponte J, Desai JB et al. (2004) Comparison of structured use of routine laboratory tests or near-patient assessment with clinical judgement in the management of bleeding after cardiac surgery. Br J Anaesth 92: 178-186.

16. Garvin S, Fitzgerald DC, Despotis G, Shekar P, Body SC (2010) Heparin concentration-based anticoagulation for cardiac surgery fails to reliably predict heparin bolus dose requirements. Anesth Analg 111: 849-855.

17. Shore-Lesserson L (2005) Evidence based coagulation monitors: heparin monitoring, thromboelastography, and platelet function. Semin Cardiothorac Vasc Anesth 9: 41-52.

18. Hardy JF, Belisle S, Robitaille D, Perrault J, Roy M, et al. (1996) Measurement of heparin concentration in whole blood with the Hepcon/HMS device does not agree with laboratory determination of plasma heparin concentration using a chromogenic substrate for activated factor X. J Thorac Cardiovasc Surg 112: 154-161.

19. Hemker HC, Beguin S (1993) Standard and method independent units for heparin anticoagulant activities. Thromb Haemost 70: 724-728.

20. Al Dieri R, Alban S, Beguin S, Hemker HC (2004) Thrombin generation for the control of heparin treatment, comparison with the activated partial thromboplastin time. J Thromb Haemost 2: 1395-1401. 
21. de Moerloose P (1996) Laboratory evaluation of hemostasis before cardiac operations. Ann Thorac Surg 62: 1921-1925.

22. Tanaka KA, Key NS, Levy JH (2009) Blood coagulation: hemostasis and thrombin regulation. Anesth Analg 108: 1433-1446.

23. Hoffman M, Monroe DM (2007) Coagulation 2006: a modern view of hemostasis. Hematol Oncol Clin North Am 21: 1-11.

24. Levy JH, Dutton RP, Hemphill JC, Shander A, Cooper D, et al. (2010) Multidisciplinary approach to the challenge of hemostasis. Anesth Analg 110: 354-364.

25. Chee YL, Crawford JC, Watson HG, Graeves M (2008) Guidelines on the assessment of bleeding risk prior to surgery or invasive procedures. British Committee for Standards in Haematology. Br J Haematol 140: 496-504.

26. Gelb AB, Roth RI, Levin J, London MJ, Noall RA, et al. (1996) Changes in blood coagulation during and following cardiopulmonary bypass: lack of correlation with clinical bleeding. Am J Clin Pathol, 106: 8799.

27. Gravlee GP, Arora S, Lavender SW, Mills SA, Hudspeth AS, et al. (1994) Predictive value of blood clotting tests in cardiac surgical patients. Ann Thorac Surg 58: 216-221.

28. Hattersley PG (1966) Activated coagulation time of whole blood. Jama 196: 436-440.

29. Bull BS, Korpman RA, Huse WM, Briggs BD (1975) Heparin therapy during extracorporeal circulation. I. Problems inherent in existing heparin protocols. J Thorac Cardiovasc Surg 69: 674-684.

30. Garvin S, Fitzgerald D, Muehlschlegel JD, Perry TE, Fox AA, et al. (2010) Heparin dose response is independent of preoperative antithrombin activity in patients undergoing coronary artery bypass graft surgery using low heparin concentrations. Anesth Analg 111: 856-861.

31. Lemmer JH Jr, Despotis GJ (2002) Antithrombin III concentrate to treat heparin resistance in patients undergoing cardiac surgery. J Thorac Cardiovasc Surg 123: 213-217.

32. Nicholson SC, Keeling DM, Sinclair ME, Evans RD (2001) Heparin pretreatment does not alter heparin requirements during cardiopulmonary bypass. Br J Anaesth 87: 844-847.

33. Flom-Halvorsen HI, Ovrum E, Abdelnoor M, Bjornsen S, Brosstad F (1999) Assessment of heparin anticoagulation: comparison of two commercially available methods. Ann Thorac Surg 67: 1012-1017.

34. Prisco D, Paniccia R (2003) Point-of-Care Testing of Hemostasis in Cardiac Surgery. Thromb J 1: 1.

35. Svenmarker S, Appelblad M, Jansson E, Haggmark S (2004) Measurement of the activated clotting time during cardiopulmonary bypass: differences between Hemotec ACT and Hemochron Jr apparatus. Perfusion 19: 289-294.

36. Bowers J, Ferguson JJ, 3rd (1994) The use of activated clotting times to monitor heparin therapy during and after interventional procedures. Clin Cardiol 17: 357-361.

37. Slaughter TF, LeBleu TH, Douglas JM, Leslie JB, Parker JK, et al. (1994) Characterization of prothrombin activation during cardiac surgery by hemostatic molecular markers. Anesthesiology 80: 520-526.

38. Koster A, Despotis G, Gruendel M, Fischer T, Praus M, et al. (2002) The plasma supplemented modified activated clotting time for monitoring of heparinization during cardiopulmonary bypass: a pilot investigation. Anesth Analg 95: 26-30.

39. Hemker HC, Beguin S (2000) Phenotyping the clotting system. Thromb Haemost 84: 747-751.

40. Weber CF, Zacharowski K (2012) Perioperative point of care coagulation testing. Dtsch Arztebl Int 109: 369-375.

41. Enriquez L, Shore-Lesserson L (2009) Point-of-care coagulation testing and transfusion algorithms. Br J Anaesth 103: i14-22.

42. Shore-Lesserson L, Manspeizer HE, DePerio M, Francis S, Vela-Cantos F, et al. (1999) Thromboelastography-guided transfusion algorithm reduces transfusions in complex cardiac surgery. Anesth Analg 88: 312-319.

43. Toulon P, Ozier Y, Ankri A, Fleron MH, Leroux G, et al. (2009) Point-of-care versus central laboratory coagulation testing during haemorrhagic surgery. A multicenter study. Thromb Haemost 101: 394-401. 
44. Faraday N, Guallar E, Sera VA, Bolton ED, Scharpf RB, et al. (2002) Utility of whole blood hemostatometry using the clot signature analyzer for assessment of hemostasis in cardiac surgery. Anesthesiology 96: 1115-1122.

45. Despotis G, Avidan M, Eby C (2009) Prediction and management of bleeding in cardiac surgery. J Thromb Haemost 7: 111-117.

46. Karkouti K, McCluskey SA, Syed S, Pazaratz C, Poonawala, et al. (2010) The influence of perioperative coagulation status on postoperative blood loss in complex cardiac surgery: a prospective observational study. Anesth Analg 110: 1533-1540.

47. Coakley M, Hall JE, Evans C, Duff E, Billing V, et al. (2011) Assessment of thrombin generation measured before and after cardiopulmonary bypass surgery and its association with postoperative bleeding. J Thromb Haemost 9: 282-292.

48. Pillai RC, Fraser JF, Ziegenfuss M, Bhaskar B (2014) The influence of circulating levels of fibrinogen and perioperative coagulation parameters on predicting postoperative blood loss in cardiac surgery: a prospective observational study. J Card Surg 29: 189-195.

49. Josefy S, Briones R, Bryant BJ (2011) Preoperative coagulation studies to predict blood component usage in coronary artery bypass graft surgery. Immunohematology 27: 151-153.

50. Kuepper F, Dangas G, Mueller-Chorus A, Kulka PM, Zenz M, et al. (2003) Fibrinolytic activity and bleeding after cardiac surgery with cardiopulmonary bypass and low-dose aprotinin therapy. Blood Coagul Fibrinolysis 14: 147-153.

51. Snircova J, Jares M, Maly M, Straka Z, Spegar J, et al. (2008) Postoperative blood loss in coronary surgery. No real impact of fibrinolysis detected by thromboelastography and D-dimers. A prospective, randomized study. Int Heart J 49: 25-38.

52. Hartert H (1951) Thrombelastography, a method for physical analysis of blood coagulation. Z Gesamte Exp Med 117: 189-203.

53. Luddington RJ (2005) Thrombelastography/thromboelastometry. Clin Lab Haematol, 27: 81-90.

54. Venema LF, Post WJ, Hendriks HG, Huet RC, de Wolf JT, et al. (2010) An assessment of clinical interchangeability of TEG and RoTEM thromboelastographic variables in cardiac surgical patients. Anesth Analg 111: 339-344.

55. Wasowicz M, McCluskey SA, Wijeysundera DN, Yau TM, Meinri M, et al. (2010) The incremental value of thrombelastography for prediction of excessive blood loss after cardiac surgery: an observational study. Anesth Analg 111: 331-338.

56. Ak K, Isbir CS, Tetik S, Atalan N, Tekeli A, et al. (2009) Thromboelastography-based transfusion algorithm reduces blood product use after elective CABG: a prospective randomized study. J Card Surg 24: 404-410.

57. Aoki K, Sugimoto A, Nagasawa A, Saito M, Ohzeki H (2012) Optimization of thromboelastographyguided platelet transfusion in cardiovascular surgery. Gen Thorac Cardiovasc Surg 60: 411-416.

58. Weber CF, Gorlinger K, Meininger D, Herrmann E, Bingold T, et al. (2012) Point-of-care testing: a prospective, randomized clinical trial of efficacy in coagulopathic cardiac surgery patients. Anesthesiology 117: 531-547.

59. Lee GC, Kicza AM, Liu KY, Nyman CB, Kaufman RM, et al. (2012) Does rotational thromboelastometry (ROTEM) improve prediction of bleeding after cardiac surgery? Anesth Analg 115: 499-506.

60. Theusinger OM, Schroder CM, Eismon J, Emmert MY, Seifert B, et al. (2013) The influence of laboratory coagulation tests and clotting factor levels on Rotation Thromboelastometry (ROTEM(R)) during major surgery with hemorrhage. Anesth Analg 117: 314-321.

61. Mann KG, Brummel K, Butenas S, (2003) What is all that thrombin for? J Thromb Haemost 1: 15041514.

62. Hemker HC, Giesen P, Al Dieri R, Regnault V, de Smed E, et al.(2002) The calibrated automated thrombogram (CAT): a universal routine test for hyper- and hypocoagulability. Pathophysiol Haemost Thromb 32: 249-253. 
63. Reece MJ, Klein AA, Salviz EA, Hastings A, Ashworth A, et al. (2011) Near-patient platelet function testing in patients undergoing coronary artery surgery: a pilot study. Anaesthesia 66: 97-103.

64. Velik-Salchner C, Maier S, Innerhofer P, Kolbitsch C, Streif W, et al. (2009) An assessment of cardiopulmonary bypass-induced changes in platelet function using whole blood and classical light transmission aggregometry: the results of a pilot study. Anesth Analg 108: 1747-1754.

65. Rahe-Meyer N, Winterhalter M, Boden A, Froemke C, Piepenbrock S, et al. (2009) Platelet concentrates transfusion in cardiac surgery and platelet function assessment by multiple electrode aggregometry. Acta Anaesthesiol Scand 53: 168-175.

66. Chowdhury M, Shore-Lesserson L, Mais AM, Leyvi G (2014) Thromboelastograph with Platelet Mapping(TM) predicts postoperative chest tube drainage in patients undergoing coronary artery bypass grafting. J Cardiothorac Vasc Anesth 28: 217-223.

67. Preisman S, Kogan A, Itzkovsky K, Leikin G, Raanani E (2010) Modified thromboelastography evaluation of platelet dysfunction in patients undergoing coronary artery surgery. Eur J Cardiothorac Surg 37: 1367-1374.

68. Weitzel NS, Weitzel LB, Epperson LE, Karimpour-Ford A, Tran ZV, et al. (2012) Platelet mapping as part of modified thromboelastography (TEG(R)) in patients undergoing cardiac surgery and cardiopulmonary bypass. Anaesthesia 67: 1158-1165.

69. Forestier F, Coiffic A, Mouton C, Ekouevi D, Chene G, et al. (2002) Platelet function point-of-care tests in post-bypass cardiac surgery: are they relevant? $\mathrm{Br}$ J Anaesth 89: 715-721.

70. Koestenberger M, Cvirn G, Nagel B, Rosenkranz A, Leschnik B, et al.(2008) Thrombin generation determined by calibrated automated thrombography (CAT) in pediatric patients with congenital heart disease. Thromb Res 122: 13-19.

71. Macfarlane RG, Biggs R (1953) A thrombin generation test; the application in haemophilia and thrombocytopenia. J Clin Pathol 6: 3-8.

72. Hemker HC, Giesen P, Al Dieri R, Regnault V, de Smedt E, et al. (2003) Calibrated automated thrombin generation measurement in clotting plasma. Pathophysiol Haemost Thromb 33: 4-15.

73. Hemker HC, Giesen PL, Ramjee M, Wagenvoord R, Beguin S (2000) The thrombogram: monitoring thrombin generation in platelet-rich plasma. Thromb Haemost 83: 589-591.

74. Ninivaggi M, Apitz-Castro R, Dargaud Y, de Laat B, Hemker HC, et al. (2012) Whole-blood thrombin generation monitored with a calibrated automated thrombogram-based assay. Clin Chem 58: 12521259.

75. Bosch Y, Al Dieri R, Bloemen S, de Laat B, ten Cate H, et al. (2013) Preoperative thrombin generation is predictive for the risk of blood loss after cardiac surgery: a research article. J Cardiothorac Surg 8: 154.

76. Bosch Y, Al Dieri R, ten Cate H, Nelemans P, Bloemen S, et al. (2014) Measurement of thrombin generation intra-operatively and its association with bleeding tendency after cardiac surgery. Thromb Res 133: 488-494.

77. Hayashi T, Sakurai Y, Fukuda K, Yada K, Ogiwara K, et al. (2011) Correlations between global clotting function tests, duration of operation, and postoperative chest tube drainage in pediatric cardiac surgery. Paediatr Anaesth 21: 865-871.

78. Despotis GJ, Joist JH, Goodnough LT (1997) Monitoring of hemostasis in cardiac surgical patients: impact of point-of-care testing on blood loss and transfusion outcomes. Clin Chem 43: 1684-1696. 

CHAPTER 2

AIMS AND OUTLINE OF THE THESIS 

To underlie the great need for a new hemostatic test that is able to classify patients with increased risk on thrombosis or bleeding, and to monitor heparin therapy during cardiopulmonary bypass, it was attempted to create a clear overview divided in 5 Chapters (3 till 7) focusing on several research questions:

\section{Chapter 3: How reliable is the ACT method?}

This chapter focuses on the repeatability and agreement of three different ACT analyzers: the GEM PCL (Instrumentation Laboratory), the Hemochron 801 (International Technidyne Corporation) and the ACT II Automated Coagulation Timer (Medtronic), using all three other detection methods.

Chapter 4: Is there an association between the most important thrombin inhibitor antithrombin and the ACT?

The aims of this observational study were to define the relationship between preoperative plasma antithrombin activity, ACT levels before the establishment of cardiopulmonary bypass, and heparin sensitivity index (HSI), and to identify other factors related to decreased ACT levels.

Chapter 5: Is there a relationship between conventional clotting tests and thrombin generation measured intraoperatively, and changes in hemostatic equilibrium and blood loss?

We measured thrombin generation by Calibrated Automated Thrombography (CAT) and conventional clotting tests in patients who underwent cardiac surgery with $\mathrm{CPB}$, in an attempt to describe the relationship between the changes in hemostatic equilibrium during surgery and the changes in these tests. In addition, we investigated whether thrombin generation measured intraoperatively, is associated with an increased risk of bleeding after surgery.

Chapter 6: Is thrombin generation preoperatively measured predictive for blood loss after cardiac surgery?

In this study the value of thrombin generation parameters measured by the CAT for prediction of blood loss after cardiac surgery with cardiopulmonary bypass was investigated. Thrombin generation was measured both in platelet rich and platelet poor plasma. 
Chapter 7: How does rotational thromboelastometry (ROTEM) change as result of $\mathrm{CPB}$ and is it predictive for postoperative bleeding?

This study aimed to find out whether ROTEM can identify patients with increased bleeding risk. Besides ROTEM, platelet count, fibrinogen and hematocrit were measured to characterize the alterations of hemostasis during surgery.

\section{Chapter 8: General Discussion}

All results will be discussed. 


\section{CHAPTER 3}

\section{AN EVALUATION OF FACTORS AFFECTING ACTIVATED CLOTTING TIME}

Yvonne P.J. Bosch

Patrick W. Weerwind

Patty J. Nelemans

Jos G. Maessen

Baheramsjah Mochtar

\section{Based on:}

An evaluation of factors affecting the activated coagulation time. Journal of Cardiothoracic and Vascular Anesthesia, 2012, 26 (4): 536-568. 


\section{Abstract}

Objective: Although failure to achieve an adequate activated clotting time (ACT) after full heparinization before cardiopulmonary bypass is attributed to antithrombin (AT) deficiency, it remains unclear if this is a causative mechanism of decreased heparin responsiveness. Therefore the authors determined the relationship between AT and other coagulation-related factors that affect the ACT measurement and heparin sensitivity index before establishment of cardiopulmonary bypass.

Design: Observational study.

Setting: University medical center.

Participants: Adult elective cardiac surgical patients.

Interventions: Preoperative data collection included demographics, type of preoperative medical therapy, hemoglobin, platelet count, and AT. Intraoperative measurements included ACT and anti-Xa activity.

Results: Of the 203 patients enrolled in this study, 10\% $(n=21)$ did not achieve an adequate ACT ( $\geq 400$ seconds) after full heparinization. Subnormal AT activity $(55 \%$ $79 \%$ ), was not related to low ACT and a low heparin sensitivity index. Preoperative low-molecular-weight heparin therapy did not cause a decreased ACT response. In addition, a lower anti-Xa activity was significantly associated with a lower ACT value. However, preoperative low hemoglobin levels as well as high platelet counts were identified to be significantly associated with a diminished ACT.

Conclusions: All these observations suggest that failure to achieve an adequate ACT is in general not an indicator of AT deficiency but could be affected by high platelet counts and low hemoglobin levels.

Key words: activated clotting time, antithrombin, decreased heparin responsiveness, heparin sensitivity index 


\section{Introduction}

During cardiac surgery involving cardiopulmonary bypass (CPB), a large dose of unfractionated heparin (300-400 IU/kg body weight) is used to attenuate the coagulation cascade [1]. It is imperative that adequate systemic heparinization is achieved before establishment of CPB.

Adequate anticoagulation before the establishment of $\mathrm{CPB}$ is monitored by the activated clotting time (ACT). The ACT is a rapid, real-time measurement of the coagulation potential of whole blood after activation with kaolin or celite. The ACT of normal blood (130 \pm 22 seconds) [2] is prolonged after heparinization [2-5]. An ACT > 400 seconds is widely considered to indicate adequate anticoagulation for patients undergoing CPB [1]. A lower than expected ACT after full heparinization is often termed heparin resistance, however, no universally accepted definition exists for this condition $[2,6$, 7]. The term also is misleading because there really is an increase in ACT after heparin, although less than desired [8]. A better term is decreased heparin responsiveness. Heparin responsiveness can be expressed by the heparin sensitivity index (HSI), controlling for baseline ACT, heparin dose used and body weight [9].

Multiple factors are presumed to be responsible for producing decreased heparin responsiveness, including antithrombin (AT) deficiency [10]. Heparin alone has very little anticoagulant activity, and in plasma, it acts by increasing the intrinsic anticoagulant activity of the serine protease inhibitor AT [11, 12]. Therefore, effective heparin anticoagulation, in general requires sufficient levels of plasma AT [13, 14]. The association between AT activity and ACT is complicated by other preoperative factors that affect the ACT, among which are preoperative heparin use, platelet counts, platelet function and other anticoagulants $[2,7,10,15,16]$.

The aims of this observational study was to define the relationship between preoperative plasma AT activity, and ACT levels before establishment of CPB, and HSI, and to identify other factors related to decreased ACT levels.

\section{Methods}

Over a 6-month period, adult patients undergoing elective cardiac operations were observed in this study. Institutional review board approval was received for this evaluation. Because this quality control evaluation did not influence the frequency or volume of blood withdrawn from the patient or the routine care of the patient, informed consent was considered unnecessary.

Preoperative data collection included demographics, type of preoperative anticoagulant or platelet antiaggregant therapy, and statins. Platelet count, hemoglobin 
(Coulter LH750 hematology analyzer, Beckman Coulter Inc. Miami, USA), and AT activity (Siemens Berichrom Antithrombin III kit, Marburg, Germany) were measured the day before surgery. Kaolin-activated ACT (Hemotec ACT II Automated Coagulation Timer, Medtronic, Inc. Minneapolis, MN) was measured before and 5 minutes after administration of systemic heparinization. The HSI was calculated from the change in ACT from before to after heparin administration, divided by the heparin dose, per kilogram of body weight [17]. In addition, anti-Xa activity was measured (Biophen Heparin (LRT), Hyphen Biomed, Neuville sur Oise, France) after heparinization but before CPB. Plasma was diluted with normal pool plasma, and an universal calibrator was used (Biophen Heparin Calibrator, Hyphen Biomed, Neuville sur Oise, France). The antiXa activity was corrected for hemodilution.

General anaesthesia was induced using weight related dosing of sufentanil and etomidate, and muscle relaxation was achieved with pancuronium bromide. General anaesthesia was maintained during surgery using propofol. An initial dose of heparin, $300 \mathrm{IU} / \mathrm{kg}$ of body weight, (LEO Pharmaceutical Products, Ballerup, Denmark) was injected into a central venous catheter before the initiation of CPB. Five minutes after this loading dose, the ACT was measured and, if the value was $\geq 400$ seconds, CPB was initiated. If necessary, additional heparin was added to achieve an ACT of $>400$ seconds. Patients who failed to achieve an ACT of $\geq 400$ seconds after full heparinization were assigned to the low ACT (L-ACT) group. Patients with an adequate ACT ( $\geq 400$ seconds) were assigned to the high-ACT (H-ACT) group. At the end of CPB, heparin was reversed by protamine chloride (Valeant Pharmaceuticals, Eschborn, Germany) at a 1:1 ratio of the total loading dose before $\mathrm{CPB}$.

The L-ACT group and the H-ACT group were compared for differences in variables that may have been associated with decreased ACT levels. Categorical data were expressed as proportions and tested for statistical significance using the Pearson $\chi^{2}$ test; continuous variables were expressed as mean \pm standard deviation and tested with Student's $t$-test for independent samples. Furthermore, correlations among continuous variables were evaluated using the Pearson correlation coefficient. Multivariate logistic regression analysis was used to evaluate the independent effect of preoperative factors that may affect ACT levels. Success or failure to achieve an ACT of > 400 seconds was the dependent variable. Pre-operative factors that differed significantly between the L-ACT and the H-ACT group were entered as independent variables. A $p$-value $<0.05$ was considered to be significant. Data analysis was performed using SPSS 15.0 for windows (SPSS Inc, Chicago, IL). 


\section{Results}

In total 203 patients were enrolled in this study. Patients $(n=21)$ who did not achieve an initial ACT of $\geq 400$ seconds after full heparinization were assigned to the L-ACT group. The H-ACT group consisted of 182 patients. The demographic characteristics and preoperative data on the medication of the patients are presented in Table 1. Anticoagulant/antiaggregant therapy was categorized into 4 groups: 1) no anticoagulant medication, 2) aspirin and/or clopidogrel, 3) low-molecular-weight heparin (LMWH), and 4) coumarin derivatives.

No significant differences existed in these characteristics between the L-ACT and the H-ACT group. Because all patients with coumarin therapy were in the H-ACT group, and because of the influence of coumarin derivatives on the coagulation time, an additional analysis excluding the patients who were on preoperative coumarin therapy was performed. The regression coefficients of the multivariate analysis were very similar before and after excluding those patients (data not shown). Coumarin therapy showed not to be a disturbing factor in this analysis, so these patients were not excluded.

The differences in the results from preoperative routine laboratory tests and the baseline ACT between the groups are presented in Table 2. No difference in mean baseline AT activity was observed between the L-ACT (102\%) and the H-ACT (99\%) groups $(p=0.215)$. Eight patients $(3.9 \%)$ had measured subnormal AT activity (55\%$79 \%)$. These patients were in the H-ACT group. Compared with patients in the H-ACT group, those in the L-ACT group had a significantly larger platelet count preoperatively, and a significantly lower mean hemoglobin level. In the entire study population, 16 patients were identified with a platelet count $>350,000 / \mathrm{mm}^{3}(29 \%$ of the patients in the L-ACT group and $5 \%$ of the patients in the H-ACT group).

All patients were administered the same initial dose of heparin per $\mathrm{kg}$ of bodyweight. Because of the inadequate response in the L-ACT group, the mean total amount of heparin administered in this group was significantly higher than in the $\mathrm{H}$ ACT group. Anti-Xa as a measurement of heparin activity, was significantly lower in the L-ACT group after the administration of the initial dose of heparin (Table 3). 
CHAPTER 3

Table 1: Comparison of patient characteristics and preoperative anticoagulation between groups.

\begin{tabular}{llll}
\hline Variable & L-ACT $(\mathrm{n}=21)$ & H-ACT $(\mathrm{n}=182)$ & $P$ - Value \\
\hline Age $(\mathrm{y})$ & $66 \pm 11$ & $66 \pm 10$ & 0.737 \\
Weight $(\mathrm{kg})$ & $77 \pm 12$ & $81 \pm 13$ & 0.208 \\
Male/female (\% female) & $16: 5(31.3 \%)$ & $141: 41(29.1 \%)$ & 0.894 \\
Preoperative anticoagulant/antiaggregant therapy* & & & \\
No medication & $5(23.8 \%)$ & $34(18.9 \%)$ & 0.595 \\
Aspirin/Clopidogrel & $16(76.2 \%)$ & $135(74.2 \%)$ & $17(9.3 \%)$ \\
$\quad$ LMWH & $1(4.8 \%)$ & $10(5.5 \%)$ & 0.894 \\
$\quad$ Coumarins & $0(0.0 \%)$ & $141(77.5 \%)$ & \\
Preoperative statin therapy & $16(76.2 \%)$ & $141 \%$ & \\
\hline
\end{tabular}

Abbreviations: L-ACT=low activated coagulation time; H-ACT=high activated clotting time; LMWH=low molecular weight heparin. * not discontinued earlier than 5 days preoperative

Table 2: Comparison of baseline measurements between groups.

\begin{tabular}{|c|c|c|c|}
\hline Variable & L-ACT $(n=21)$ & $\mathrm{H}-\mathrm{ACT}(\mathrm{n}=182)$ & $P$ - Value \\
\hline Platelet count $\left(\times 1000 / \mathrm{mm}^{3}\right)$ & $290 \pm 75$ & $250 \pm 65$ & 0.009 \\
\hline Antithrombin (\%) & $102 \pm 8$ & $99 \pm 12$ & 0.215 \\
\hline Hemoglobin (mmol/L) & $8.1 \pm 1.2$ & $8.7 \pm 0.9$ & 0.011 \\
\hline Baseline ACT (s) & $133 \pm 13$ & $139 \pm 17$ & 0.137 \\
\hline
\end{tabular}

Abbreviations: $A C T=$ activated clotting time; $\mathrm{L}-\mathrm{ACT}=\mathrm{low}$ activated coagulation time; $\mathrm{H}-\mathrm{ACT}=$ high activated clotting time

Table 3: Comparison of intraoperative characteristics between groups.

\begin{tabular}{lllc}
\hline Variable & L-ACT $(\mathrm{n}=21)$ & H-ACT $(\mathrm{n}=182)$ & $P$ - Value \\
\hline Heparin (IU/kg) & & & 0.775 \\
$\quad$ Initial & $315 \pm 42$ & $313 \pm 34$ & $<0.001$ \\
$\quad$ Total & $398 \pm 65$ & $315 \pm 35$ & $<0.001$ \\
ACT post heparin (s) & $372 \pm 28$ & $534 \pm 104$ & $<0.001$ \\
HSI (s/lU/kg) & $0.76 \pm 0.14$ & $1.27 \pm 0.31$ & 0.02 \\
Anti-Xa (U/ml) & $8.1 \pm 0.9$ & $8.7 \pm 1.5$ & \\
\hline
\end{tabular}

Abbreviations: $A C T=$ activated clotting time; $\mathrm{L}-\mathrm{ACT}=$ low activated coagulation time; $\mathrm{H}-\mathrm{ACT}=$ high activated coagulation time; $\mathrm{HSI}=$ heparin sensitivity index 
Table 4 presents a weak association between post-heparin ACT and the preoperative AT activity ( $r=0.055)$. The ACT tended to increase at lower platelet counts and at higher hemoglobin levels ( $r=-0.296$ and $r=0.106$, respectively). Anti-Xa activity showed a positive association with the post-heparin ACT ( $r=0.263)$. To correct for baseline ACT, heparin dosage and body weight, the correlation with HSI also was determined.

Table 4: Correlation coefficients between activated clotting time and heparin sensitivity index.

\begin{tabular}{lll}
\hline & \multicolumn{2}{l}{ Pearson Correlation $(r)$} \\
\cline { 3 - 3 } Variable & ACT after heparin & $\mathrm{HSI}$ \\
\hline Preoperative & & \\
Platelet count $\left(\mathrm{x} 1000 / \mathrm{mm}^{3}\right)$ & -0.296 & -0.308 \\
Antithrombin $(\%)$ & 0.055 & -0.068 \\
Hemoglobin $(\mathrm{mmol} / \mathrm{L})$ & 0.106 & 0.110 \\
Intraoperative & & \\
Baseline ACT (s) & 0.272 & 0.152 \\
Anti-Xa (U/ml) & 0.263 & 0.183 \\
\hline
\end{tabular}

Abbreviations: $\mathrm{ACT}=$ activated clotting time; $\mathrm{HSI}=$ heparin sensitivity index

The correlations of the preoperative variables with HSI were almost equal and even changed to inverse in the case of AT compared with those with post-heparin ACT. The correlations of the intraoperative variables were weaker using HSI. Platelet count showed the highest association with heparin responsiveness. The associations among AT activity ( $r=-0.068)$, preoperative platelet count $(r=-0.308)$ and hemoglobin $(r=0.110)$ and $\mathrm{HSI}$ are presented in Figure 1-3. HSI seemed to decrease with higher platelet counts, and to increase with higher hemoglobin levels.

Table 5: Multivariate logistic regression analysis.

\begin{tabular}{|c|c|c|c|c|}
\hline Variable & B & SE & $95 \%$ C.I. for B & $P$ - Value \\
\hline Platelet count $\left(\times 1000 / \mathrm{mm}^{3}\right)$ & -0.006 & 0.003 & -0.013 to 0.001 & 0.071 \\
\hline Antithrombin (\%) & -0.013 & 0.021 & -0.056 to 0.029 & 0.540 \\
\hline Hemoglobin (mmol/L) & 0.421 & 0.227 & -0.023 to 0.865 & 0.063 \\
\hline
\end{tabular}

Abbreviations: $\mathrm{B}=$ regression coefficient; $\mathrm{C} . \mathrm{I}=$ =confidence interval; $\mathrm{SE}=$ standard error

The preoperative variables platelet count, AT activity and hemoglobin level were entered into a multivariate logistic regression analysis (Table 5). After adjustment for platelet count and hemoglobin level, there was still no association between AT and $A C T$. The results from the multivariate analysis also showed that the platelet count is still lower in the H-ACT group than in the L-ACT group, whereas the hemoglobin level 
was higher in the H-ACT group than in the L-ACT group. However, these differences were no longer statistically significant.

\section{Discussion}

The results indicated that decreased heparin responsiveness after the administration of $300 \mathrm{IU} / \mathrm{kg}$ was not associated with subnormal AT activity. Failure to achieve an adequate heparin response was associated with a lower hemoglobin and a higher preoperative platelet count, although the results were not statistically significant after mutual adjustment in a multivariate regression analysis. In addition, patients with a decreased ACT value had significantly lower anti-Xa activity despite the equal dosing of heparin.

Decreased heparin responsiveness often is attributed to AT deficiency, because the primary mechanism of action of heparin is to facilitate the enzymatic activity of AT $[6,7,10,18]$. However, in the present study, there was an apparent lack of correlation between HSI and AT activity ( $r=-0.068)$. Patients in the L-ACT group even had a higher, but not statistically significant, AT activity than patients in the H-ACT group. In the LACT group no patients had a subnormal AT activity $(<80 \%)$. These findings agree with results of other research groups [18-20]. In an in vitro study by Butenas et al. [21] the influence of alterations in plasma (anti)coagulation factor levels on the generation of thrombin in non-anticoagulated blood was investigated.

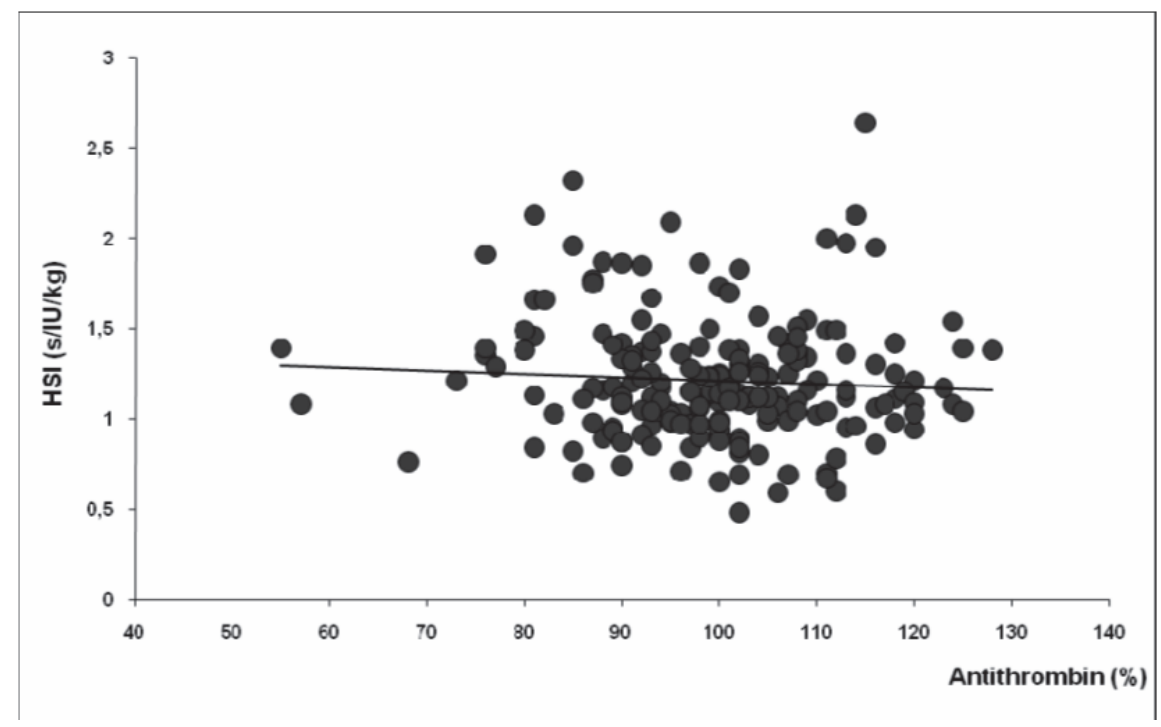

Figure 1: Association between the heparin sensitivity index (HSI) and preoperative antithrombin $(r=-0.068)$. 
In contrast to the in vivo studies, they showed that significant decreases in AT can increase thrombin generation significantly. Decreases in other coagulation inhibitors did not affect coagulation, thus emphasizing the importance of AT as an anticoagulant. Therefore, insufficient AT levels should cause inadequate anticoagulation with heparin, but this is not shown by the ACT test. A possible explanation could be the fact that the ACT is a whole blood coagulation assay, representing the interaction of plasma coagulation components, platelets and red blood cells [8]. Thus, it will not be surprising that the influence of AT alone on the ACT is not always observed, e.g. with excessive amounts of procoagulant factors or with factor deficiencies. In addition, the ACT is a measurement of the initiation phase of coagulation [22]. Because most of the thrombin (>95\%) is generated after the moment of coagulation [22], AT is consumed during the production phase of coagulation. A deficit in AT becomes more important as more AT is consumed, i.e. toward the end of the production phase, rather than in the initiation phase. The heparin response includes not only the coagulation time but also the total amount of thrombin. Furthermore, the ACT is a measurement of the contact pathway, providing no useful information about the inhibition of the extrinsic pathway triggered by factor VIla and tissue factor (TF) [23].

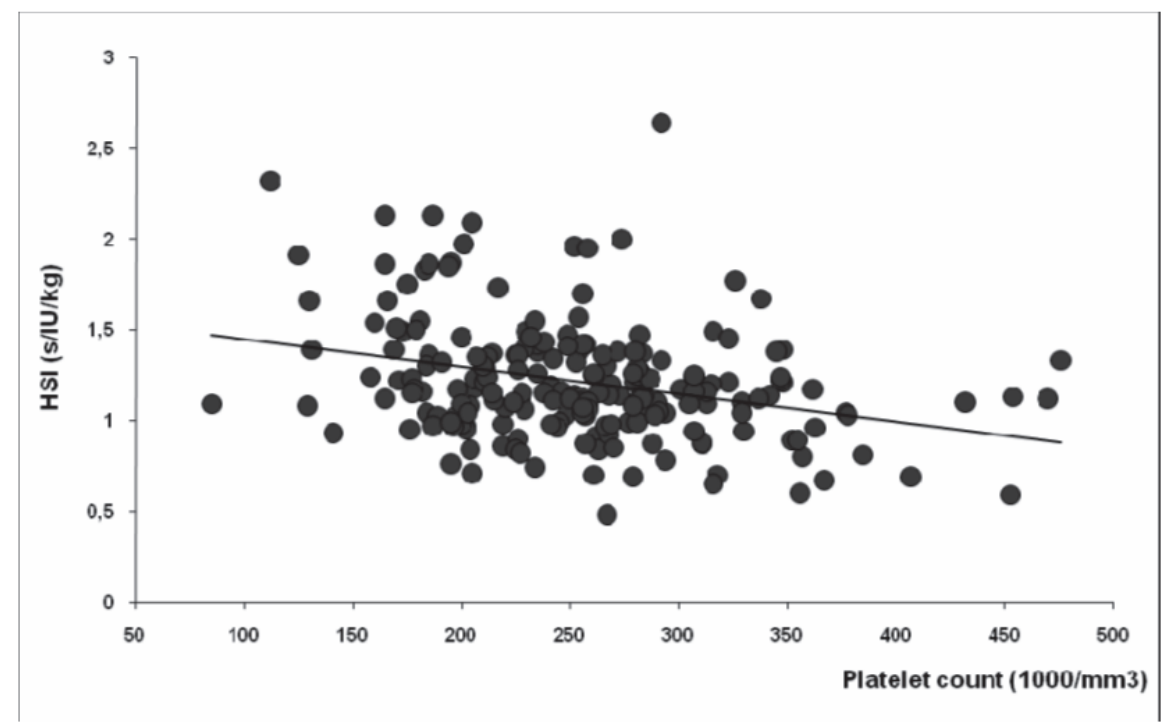

Figure 2: Association between the heparin sensitivity index (HSI) and preoperative platelet count ( $r=-0.308)$. 


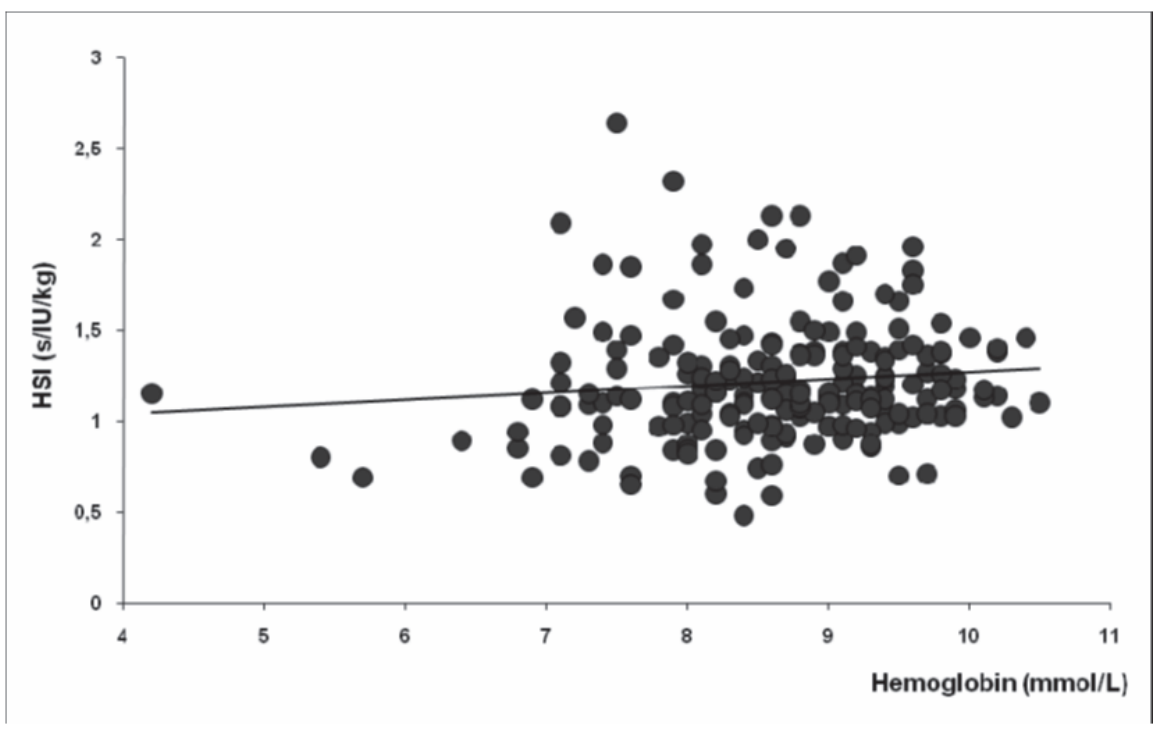

Figure 3: Association between the heparin sensitivity index (HSI) and preoperative hemoglobin $(r=0.110)$.

Moreover, there is a wide variability in heparin response, caused by AT independent mechanisms, that could affect the ACT. This variability could be attributable to factors such as the incorrect estimation of blood volume, the extravascular sequestration of heparin, plasma protein binding and the effects of the release of TF pathway inhibitor (TFPI) by heparin [24]. TFPI is recognized as a major physiological anticoagulant, because it inhibits the TF-factor VII complex, the primary initiator of coagulation in vivo. TFPI is released in high concentrations from the vascular endothelium after the injection of heparin. In diluted prothrombin-time based assays, released TFPI contributes to approximately one third of the anticoagulant effect of heparin, with the remaining being accounted for by AT [25]. AT and TFPI cooperate to control the activation of factor $X$ by the TF pathway $[25,26]$. In contrast to the prothrombin time, the ACT involves activation of the contact pathway. Therefore, the levels of TF and TFPI, and the cooperation between AT and TFPI after heparin injection probably will not be reflected accurately by the ACT.

The hypothesis that preoperative heparin treatment is associated with decreased heparin responsiveness could not be corroborated by the present findings. The present results showed that the prevalence of patients who were exposed to preoperative LMWH use was 2 times as much in the H-ACT group. In other words, in the present study population preoperative LMWH therapy was not a cause of the decreased ACT response. Preoperative treatment with heparin or LMWH should shorten the half-life of AT and, thus decrease its activity [27-29]. In the present study population, none of 
the 8 patients with a subnormal AT activity had a history of heparin or LMWH therapy. A study by Linden et al. [30] showed that a decreased response to heparin was more common in patients who had received preoperative therapeutic heparin, but this was not related causally to the decreased AT.

Because statins have, in addition to their effects in decreasing cholesterol biosynthesis, significant antithrombotic effects by decreasing platelet activation [31], this preoperative treatment was taken into account. There was no difference in the prevalence of patients who had statin therapy preoperatively between the L-ACT and H-ACT group.

The present study confirmed that high platelet counts are associated with reduced heparin responsiveness [7, 32,33]. Contact activation of platelets results in the release of platelet factor 4 which neutralizes heparin [34, 35]. With high platelet counts $\left(>300,000 / \mathrm{mm}^{3}\right)$ heparin anticoagulation will be less effective. In addition to the neutralizing effect of platelet factor 4 , platelets themselves, both activated and nonactivated, bind heparin [36], decreasing the amount of free heparin available for binding AT. The concentration of heparin required to inhibit or delay coagulation is related directly to the number of platelets [37]. This agrees with the results of Gayoso [33], who found that mean ACT levels after the administration of heparin were significantly lower in patients with thrombocytosis and that these patients required more additional heparin on CPB to maintain the ACT > 480 seconds. In the present study population, patients in the L-ACT group had significantly higher mean platelet count preoperatively than patients in the H-ACT group in the univariate analysis. Although platelet count is associated with heparin responsiveness, the statistical significance disappeared after its inclusion in a multivariate logistic regression analysis.

The present finding that the mean hemoglobin level in the L-ACT group was lower than in the H-ACT group is contradictory to a recent study of Roeloffzen et al. [38], who studied the effect of progressive anemia on the coagulation profile. They observed that a decreasing hemoglobin level was associated with a prolongation of the reaction time in a thromboelastography assay, suggesting a delay in the initiation of the coagulation cascade. This should be in accordance with a prolonged ACT value. This association was not observed in the present study. In contrast, preoperative hemoglobin was significantly lower in the L-ACT group than in the H-ACT group. The underlying mechanism of lower hemoglobin levels leading to lower ACT values is unclear. The activation of blood in the surgical wound may lead to the destruction of red blood cells and the activation of coagulation, resulting in lower hemoglobin levels and lower ACT values. However, whether this phenomenon would occur so early in the procedure before CPB and the reinfusion of shed blood, makes this explanation unlikely. Alternatively, this may be a failure of the ACT assay. Further studies may be necessary to elucidate this phenomenon. 
The ACT, measuring the anticoagulation effect of heparin, was compared with anti-Xa activity. The anti-Xa measurement is the standard test to monitor heparin levels in patients treated with heparin. The ACT values should correlate well with laboratory-derived heparin concentration values in the period before CPB [3]. In the present study, the anti-Xa level was indeed significantly lower in patients with low ACT values. However, there was only a weak correlation between the anti-Xa and the ACT. This can be explained by the fact that the relation between heparin concentration and coagulation time is nonlinear $[39,40]$. A disadvantage of the anti-Xa assay used in the present study is that a decreased heparin response caused by low AT levels probably will not be detected; in this assay, purified AT is added to the test plasma to bind all the heparin in the sample. Factor Xa (in excess) then is neutralized by the heparin-AT complex. Hence, the ACT and the anti-Xa assay are unreliable in reflecting the influence of AT. When using the anti-Xa assay as a test to monitor heparin levels, it should be taken into account that this test measures only heparin concentration and does not reflect its activity, i.e. thrombin inhibition [41].

A potential risk factor for a decreased heparin response include age $>65$ years, because of increased factor VIII and von Willebrand factor coagulation activity [7]. We could not confirm age as a risk factor.

Because the sample size was based on feasibility considerations using the available data during a 6-month time period, patients with extremely low AT activity were not identified. The L-ACT group was relatively small (10\%), and the results might have been different if more patients were with an AT activity $<80 \%$ had been included and if this decreased AT activity had been more pronounced. In addition, the authors did not measure the binding of heparin to plasma proteins, which would have provided more insight into the individual concentrations of coagulation factors.

In conclusion, monitoring anticoagulation exclusively with the ACT test may be misleading. A failure to achieve an adequate ACT is in general not an indicator of AT deficiency, which is presumed to be of significant influence, but could be affected by high platelet counts and low hemoglobin levels. 


\section{References}

1. Brister, S.J., F.A. Ofosu, and M.R. Buchanan, Thrombin generation during cardiac surgery: is heparin the ideal anticoagulant? Thromb Haemost, 1993. 70(2): p. 259-262.

2. Levy, J.H., et al., The in vitro effects of antithrombin III on the activated coagulation time in patients on heparin therapy. Anesth Analg, 2000. 90(5): p. 1076-1079.

3. Despotis, G.J., et al., Comparison of activated coagulation time and whole blood heparin measurements with laboratory plasma anti-Xa heparin concentration in patients having cardiac operations. J Thorac Cardiovasc Surg, 1994. 108(6): p. 1076-82.

4. Hattersley, P.G., Activated coagulation time of whole blood. Jama, 1966. 196(5): p. 436-440.

5. Niles, S.D., et al., Correlation of ACT as measured with three commercially available devices with circulating heparin level during cardiac surgery. J Extra Corpor Technol, 1995. 27(4): p. 197-200.

6. Avidan, M.S., et al., Recombinant human antithrombin III restores heparin responsiveness and decreases activation of coagulation in heparin-resistant patients during cardiopulmonary bypass. J Thorac Cardiovasc Surg, 2005. 130(1): p. 107-113.

7. Ranucci, M., et al., Predictors for heparin resistance in patients undergoing coronary artery bypass grafting. Perfusion, 1999. 14(6): p. 437-442.

8. Levy, J.H., Heparin resistance and antithrombin: should it still be called heparin resistance? J Cardiothorac Vasc Anesth, 2004. 18(2): p. 129-30.

9. Bar-Yosef, S., et al., Preoperative low molecular weight heparin reduces heparin responsiveness during cardiac surgery. Can J Anaesth, 2007. 54(2): p. 107-13.

10. Despotis, G.J., et al., Antithrombin III during cardiac surgery: effect on response of activated clotting time to heparin and relationship to markers of hemostatic activation. Anesth Analg, 1997. 85(3): p. 498-506.

11. Huntington, J.A., Molecular recognition mechanisms of thrombin. J Thromb Haemost, 2005. 3(8): p. 1861-1872.

12. Lane, D.A., H. Philippou, and J.A. Huntington, Directing thrombin. Blood, 2005. 106(8): p. 2605-2612.

13. Hashimoto, K., et al., Heparin and antithrombin III levels during cardiopulmonary bypass: correlation with subclinical plasma coagulation. Ann Thorac Surg, 1994. 58(3): p. 799-804; discussion 804-805.

14. Ranucci, M., et al., Determinants of antithrombin consumption in cardiac operations requiring cardiopulmonary bypass. Perfusion, 2004. 19(1): p. 47-52.

15. Esposito, R.A., et al., The role of the activated clotting time in heparin administration and neutralization for cardiopulmonary bypass. J Thorac Cardiovasc Surg, 1983. 85(2): p. 174-85.

16. Shore-Lesserson, L., et al., Anticoagulation for cardiac surgery in patients receiving preoperative heparin: use of the high-dose thrombin time. Anesth Analg, 2000. 90(4): p. 813-8.

17. Dietrich, W., et al., Low preoperative antithrombin activity causes reduced response to heparin in adult but not in infant cardiac-surgical patients. Anesth Analg, 2001. 92(1): p. 66-71.

18. Garvin, S., et al., Heparin dose response is independent of preoperative antithrombin activity in patients undergoing coronary artery bypass graft surgery using low heparin concentrations. Anesth Analg, 2010. 111(4): p. 856-61.

19. Lemmer, J.H., Jr. and G.J. Despotis, Antithrombin III concentrate to treat heparin resistance in patients undergoing cardiac surgery. J Thorac Cardiovasc Surg, 2002. 123(2): p. 213-7.

20. Nicholson, S.C., et al., Heparin pretreatment does not alter heparin requirements during cardiopulmonary bypass. Br J Anaesth, 2001. 87(6): p. 844-847.

21. Butenas, S., C. van't Veer, and K.G. Mann, "Normal" thrombin generation. Blood, 1999. 94(7): p. 216978.

22. Hemker, H.C., R. Al Dieri, and S. Beguin, Thrombin generation assays: accruing clinical relevance. Curr Opin Hematol, 2004. 11(3): p. 170-5. 
23. Koster, A., et al., The plasma supplemented modified activated clotting time for monitoring of heparinization during cardiopulmonary bypass: a pilot investigation. Anesth Analg, 2002. 95(1): p. 26-30, table of contents.

24. Garvin, S., et al., Heparin concentration-based anticoagulation for cardiac surgery fails to reliably predict heparin bolus dose requirements. Anesth Analg, 2010. 111(4): p. 849-55.

25. Sandset, P.M., B. Bendz, and J.B. Hansen, Physiological function of tissue factor pathway inhibitor and interaction with heparins. Haemostasis, 2000. 30 Suppl 2: p. 48-56.

26. Brodin, E., et al., Regulation of thrombin generation by TFPI in plasma without and with heparin. Transl Res, 2009. 153(3): p. 124-31.

27. Despotis, G.J., et al., Anticoagulation monitoring during cardiac surgery: a review of current and emerging techniques. Anesthesiology, 1999. 91(4): p. 1122-51.

28. Hirsh, J. and R. Raschke, Heparin and low-molecular-weight heparin: the Seventh ACCP Conference on Antithrombotic and Thrombolytic Therapy. Chest, 2004. 126(3 Suppl): p. 188S-203S.

29. Weitz, J.I., Low-molecular-weight heparins. N Engl J Med, 1997. 337(10): p. 688-98.

30. Linden, M.D., et al., Decreased concentration of antithrombin after preoperative therapeutic heparin does not cause heparin resistance during cardiopulmonary bypass. J Cardiothorac Vasc Anesth, 2004. 18(2): p. 131-135.

31. Colli, S., J.P. Werba, and E. Tremoli, Statins in atherothrombosis. Semin Vasc Med, 2004. 4(4): p. 40715.

32. Chan, T., N.C. Hwang, and C.H. Lim, A statistical analysis of factors predisposing patients to heparin resistance. Perfusion, 2006. 21(2): p. 99-103.

33. Gayoso, J.M., 5-year incidence of thrombocytosis and the effect on heparin dose response and heparin requirements. J Extra Corpor Technol, 1999. 31(4): p. 184-90.

34. Lane, D.A., et al., Anticoagulant activities of heparin oligosaccharides and their neutralization by platelet factor 4. Biochem J, 1984. 218(3): p. 725-732.

35. Lane, D.A., et al., Neutralization of heparin-related saccharides by histidine-rich glycoprotein and platelet factor 4. J Biol Chem, 1986. 261(9): p. 3980-3986.

36. Horne, M.K., 3rd and E.S. Chao, Heparin binding to resting and activated platelets. Blood, 1989. 74(1): p. 238-43.

37. Conley, C.L., R.C. Hartmann, and J.S. Lalley, The relationship of heparin activity to platelet concentration. Proc Soc Exp Biol Med, 1948. 69(2): p. 284-7.

38. Roeloffzen, W.W., et al., Effects of red blood cells on hemostasis. Transfusion, 2010. 50(7): p. 1536-44.

39. Despotis, G.J., et al., Response of kaolin ACT to heparin: evaluation with an automated assay and higher heparin doses. Ann Thorac Surg, 1996. 61(3): p. 795-9.

40. Shapiro, G.A., S.W. Huntzinger, and J.E. Wilson, 3rd, Variation among commercial activated partial thromboplastin time reagents in response to heparin. Am J Clin Pathol, 1977. 67(5): p. 477-80.

41. Hemker, H.C. and S. Beguin, Standard and method independent units for heparin anticoagulant activities. Thromb Haemost, 1993. 70(5): p. 724-8. 


\section{CHAPTER 4}

\section{COMPARISON OF ACT POINT-OF-CARE MEASUREMENTS: REPEATABILITY AND AGREEMENT}

Yvonne P.J. Bosch

Y.M. Ganushchak

D.S. de Jong

Based on:

Comparison of ACT point-of-care measurements: repeatability and agreement. Perfusion (2006) 21, 27-31. 


\section{Abstract}

Background: Accurate control of heparin anticoagulation is necessary during all stages of cardiopulmonary bypass. The activated clotting time (ACT), first described by Hattersley in 1966, is mostly used for determination of anticoagulation. Either celite or kaolin is used as activator. An ACT value of $480 \mathrm{sec}$ is proposed to be the safe minimum level for anticoagulation during cardiopulmonary bypass. This study was designed to determine if the ACT values of each analyzer separately are repeatable, and to determine whether there exists a significant difference in ACT values measured by three different analyzers: the GEM PCL (Instrumentation Laboratory), the Hemochron 801 (International Technidyne Corporation) and the ACT II Automated Coagulation Timer (Medtronic).

Methods: All patients required 200-300 IU/kg heparin. Blood samples for the measurement of the ACT were taken from all patients before and after heparinization, during $\mathrm{CPB}$, and after protamine administration. All samples were measured in duplicate with the three different analyzers. To compare the ACT data, the method described by Bland and Altman was used. The Pearson correlation coefficient was used to determine whether the differences were related to the average ACTs. P-values $<0.05$ were considered to be statistically significant.

Results: The results showed that the three tested ACT analyzers met the requirements of repeatability. The Pearson correlation coefficients were too low $(-0.012$, 0.221 and -0.241 , respectively) to show any correlation between the differences and the means. The ACT values measured with the Hemochron 801 were not significantly different from the ACT values measured with the ACT II analyzer. However, the GEM PCL did not agree with the Hemochron 801: $\triangle A C T=-80.20 \pm 143.06$, or the ACT II analyzer: $\triangle A C T=-119.13 \pm 138.51(p<0.001)$. A rather strong correlation was evident between the differences and the means measured with the GEM PCL compared with the Hemochron 801 ( $r=0.68$ ) and the ACT II analyzer ( $r=0.76)$.

Conclusions: All analyzers used celite or kaolin as activator. However, it was evident that the ACT measurements depend also on the analyzer that had been chosen. A precaution that ACT values could not always be interpreted in the same way seems to be necessary. 


\section{Introduction}

Anticoagulation is necessary during various medical and surgical procedures. Blood coagulation could arise from its exposure to the foreign surfaces of the extracorporeal circuit. During cardiopulmonary bypass (CPB), anticoagulation is achieved with heparin. Heparin forms a complex with antithrombin III. This binding inhibits the coagulation factors IIa, Xa, and to a lesser degree IXa and Xla, resulting in interruption of coagulation. The use of heparin and monitoring of anticoagulation is of vital importance: overdosing with heparin can result in dangerous bleeding, whereas too low a dose of heparin can lead to thrombosis. The activated coagulation time (ACT), first described by Hattersley in 1966, is a method for monitoring the anticoagulation effects of heparin [1]. An ACT of $480 \mathrm{sec}$ is proposed to be the safe minimal level for anticoagulation during CPB. The ACT is measured by adding fresh whole blood to a test tube containing an activator. The type of activator used will affect the degree of prolongation of the ACT. Celite and kaolin are mostly used as activators.

Included in this study were three different analyzers: the GEM PCL (Instrumentation Laboratory, Lexington, MA, USA), the Hemochron 801 (International Technidyne Corporation, Edison, NJ, USA) and the ACT II Automated Coagulation Timer (Medtronic, Inc. Minneapolis, MN, USA). The aim of this study was to determine whether the ACT values of each analyzer separately are repeatable, and to determine whether there is a significant difference in ACT values measured by the three different analyzers.

\section{Materials and Methods}

Eleven patients undergoing coronary bypass or valve operations were investigated in this study. Subjects with known disorders of the clotting system and subjects using anticoagulation medications prior to surgery were excluded from participation.

Devices utilized in this study design were the GEM PCL (Instrumentation Laboratory), the Hemochron 801 (International Technidyne Corporation) and the ACT II Automated Coagulation Timer (Medtronic). These analyzers differ by activators, detection systems and use different sample volumes. During surgery, blood samples for measurement of the ACT were obtained from all patients at the following times: T1) before heparinization; T2) pre-CPB (post heparin administration); T3) 5 min after the start of CPB; T4) during CPB, 30 min after T3; T5) during CPB, 30 min after T4; and T6) post protamine administration. All samples were measured in duplicate with the three different analyzers and were run immediately. Time count was started the moment the blood came into contact with the activator. 
An adult CPB circuit was constructed for each patient utilizing a roller pump (Stöckert Instrument $\mathrm{GmbH}$, Munchen, Germany), custom pack adult (Tygon and silicon tubing, Bioline heparin coated, Jostra AG, Hirrlingen, Germany), hollow-fiber membrane oxygenator (Capiox SX18, Terumo Europe NV, Leuven, Belgium), arterial filter (Pall Biomedical Ltd, Portsmouth, UK), cardiotomy reservoir (BCR 3500 GOLD, Baxter, Bentley Division, Irvine, CA, USA), and a venous reservoir (BMR-1900G, Baxter, Bentley Division). The priming solution for CPB consisted of $1300 \mathrm{~mL}$ Haemaccel 3.5\% (Behringer AG, Marburg, Germany), $200 \mathrm{~mL}$ mannitol 20\% (Baxter B.V., Uden, the Netherlands), $100 \mathrm{~mL}$ human albumin 20\% (Sanquin CLB, Amsterdam, the Netherlands), 50mL NaHCO3 8.4\% (B. Braun, Melsungen AG, Germany), and 7500 IU heparin (Leo Pharmaceutical Products, Middlesborough, UK). The flow index was maintained ca. $2.4 \mathrm{~L} / \mathrm{min}$ per meter square during perfusion. Applied temperatures varied from 30 to $37^{\circ} \mathrm{C}$. Anticoagulation was achieved with 200-300 IU/kg heparin, and bypass was started when the ACT had reached a minimum level of 480 s. Reversal of heparin was affected with the use of protamine hydrocloride $(1.0-1.5 \mathrm{mg} / 100 \mathrm{IU}$ of the total dose of heparin) upon completion of the operative procedure.

The repeatability test and the bias (agreement) between devices were measured by the method described by Bland and Altman to compare results [2]. The one-sample t-test was used to compare differences between the data, and the Pearson correlation coefficient $(r)$ was used to determine whether the differences were related to the average ACTs. Data are generally expressed as means and standard deviations (s). Pvalues $<0.05$ were considered statistically significant. All statistical analyses were performed using SPSS 10.1 for Microsoft Windows (SPSS Inc., Chicago, IL, USA).

\section{Results}

\section{Repeatability}

In Figures 1-3, the differences between the duplicate measurements are plotted against the mean ACT. The mean difference is indicated by a horizontal line at $\triangle A C T=-$ $8.78 \pm 37.61$ (GEM PCL), at $\triangle \mathrm{ACT}=-19.77 \pm 68.82$ (Hemochron 801) and at $\triangle \mathrm{ACT}=-6.23$ \pm 39.21 (ACT II). The test indicates that the duplicate measurements do not differ significantly at $\alpha=0.05$. The $p$-values for the GEM PCL, the Hemochron 801 and the ACT II are $p=0.177,0.081$ and 0.384 , respectively. The standard deviation is highest for the Hemochron 801 analyzer, but, with a p-value of 0.081 , not significant. Also, this analyzer shows the highest variation. The 'limits of repeatability' are also shown in the figures. The values are summarized in Table 1. 
The Pearson correlation coefficient is used to determine whether the differences (bias) are related to the average ACTs. This correlation was very low for the three analyzers. The values for the GEM PCL, the Hemochron 801 and the ACT II, respectively, were $0.012,-0.221$ and 0.241 . This indicates that there is no correlation between the mean ACT and the bias.

Table 1: Repeatability of ACT analyzers and correlation between bias and average ACT.

\begin{tabular}{llllllc}
\hline & $\mathrm{n}$ & $\begin{array}{l}\text { Average difference } \\
(\mathrm{sec})\end{array}$ & $\begin{array}{l}\text { 95\% Limits of } \\
\text { repeatability }\end{array}$ & t-value & $\begin{array}{l}\mathrm{p} \text {-value } \\
\text { GEM PCL }\end{array}$ & $\begin{array}{l}\text { Pearson } \\
\text { correlation }\end{array}$ \\
Hemochron 801 & 39 & $-8.77(37.61)$ & $-82.5-64.9$ & -1.38 & 0.177 & -0.012 \\
ACT II & 31 & $-19.77(68.82)$ & $-154.7-115.1$ & -1.79 & 0.081 & -0.221 \\
\hline
\end{tabular}

Table 2: Agreement between ACT analyzers and correlation between bias and average ACT.

\begin{tabular}{lllllll}
\hline & $\mathrm{n}$ & $\begin{array}{l}\text { Average difference } \\
(\mathrm{sec})\end{array}$ & $\begin{array}{l}\text { 95\% Limits of } \\
\text { agreement }\end{array}$ & t-value & p-value & $\begin{array}{l}\text { Pearson } \\
\text { correlation }\end{array}$ \\
\hline GEM PCL - Hemochron 801 & 40 & $-80.2(143.06)$ & $-355.7-195.3$ & -3.60 & $0.001^{*}$ & 0.68 \\
GEM PCL - ACT II & 39 & $-119.13(138.51)$ & $-385.6-147.3$ & -5.47 & $<0.001^{*}$ & 0.76 \\
Hemochron 801 - ACT II & 39 & $-34.09(146.68)$ & $-305.3-237.1$ & -1.54 & 0.132 & 0.10 \\
\hline
\end{tabular}

\section{Agreement}

In Figures 4-6, the difference between the different analyzers is plotted against the mean ACT. The mean difference is indicated by a horizontal line at $\triangle \mathrm{ACT}=-80.2 \pm$ 143.06 (GEM PCL versus Hemochron 801), at $\triangle \mathrm{ACT}=-119.13 \pm 138.51$ (GEM PCL versus ACT II) and at $\triangle A C T=-34.09 \pm 146.68$ (Hemochron 801 versus ACT II). The 'limits of agreement' are also indicated with horizontal lines. The measurements show that there is a significant difference between the GEM PCL and both the Hemochron 801 ( $p$ $=0.001$ ) and the ACT II analyzer $(p<0.001$ ) (see also Table 2.). There is no agreement. The Hemochron 801 and the ACT II do not differ significantly $(p=0.132)$. The Pearson correlation coefficient shows a rather strong correlation between the difference and the mean ACT for the GEM PCL compared with the Hemochron 801 ( $r=0.68$ ) and the ACT II ( $r=0.76)$. The Pearson correlation coefficient is low for the test between the Hemochron 801 and the ACT II ( $r=0.10)$. There is almost no correlation between the difference and the mean ACT. 


\section{Discussion}

To measure ACT, various analyzers can be used. However, each analyzer has a different detection system to measure the formation of a clot. Consequently, the ACT could differ for each analyzer. That makes it important to study the repeatability and the agreement with standard analyzers before any new apparatus can be used.

The test of repeatability between the GEM PCL, the Hemochron 801 and the ACT II showed that the duplicate measurements do not differ significantly from each other: all the ACT values are repeatable.

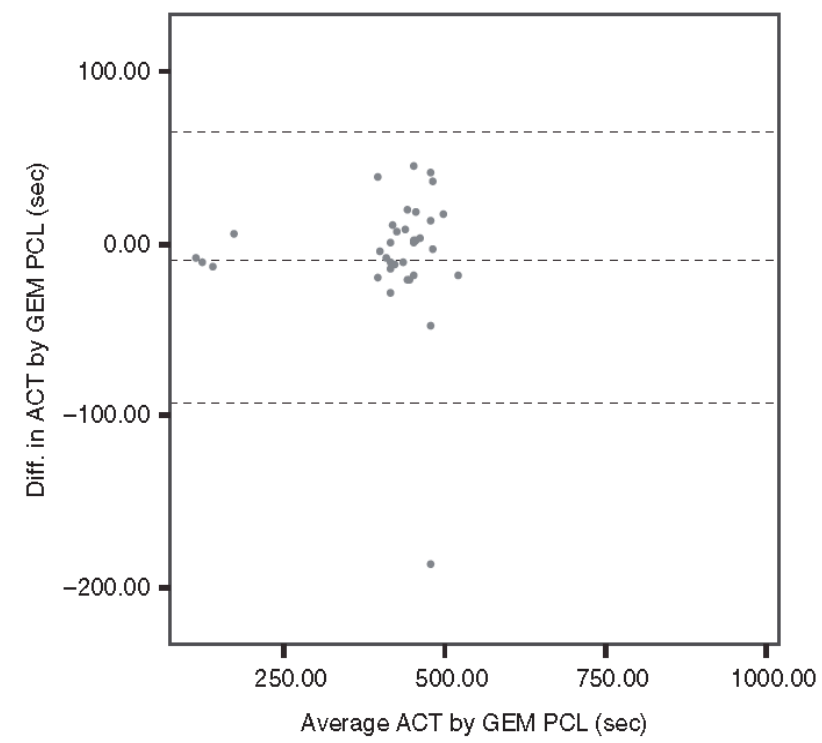

Figure 1: Repeatability of ACT measurements by GEM PCL. 


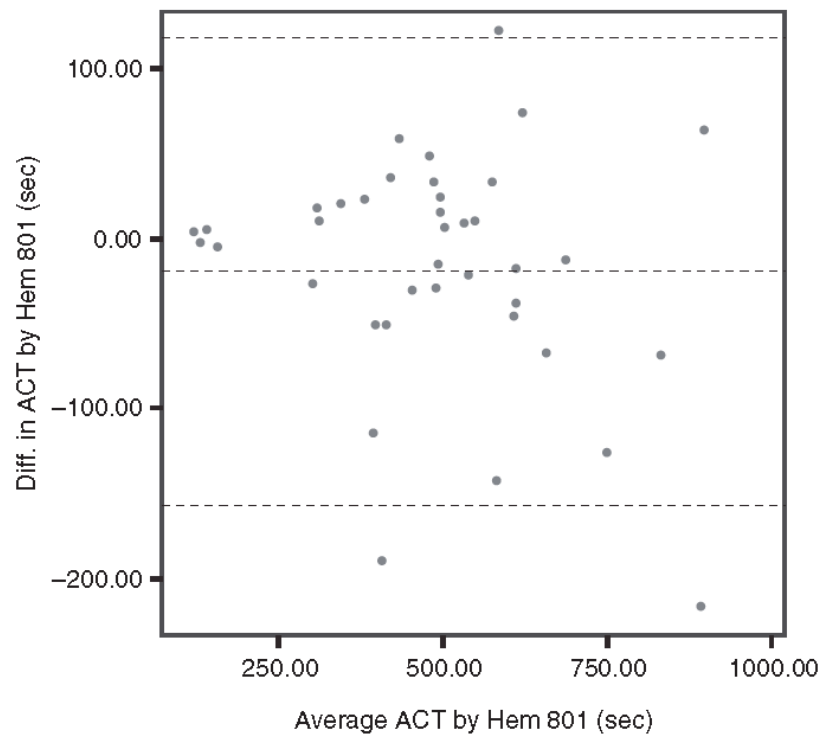

Figure 2: Repeatability of ACT Measurements by Hemochron 801.

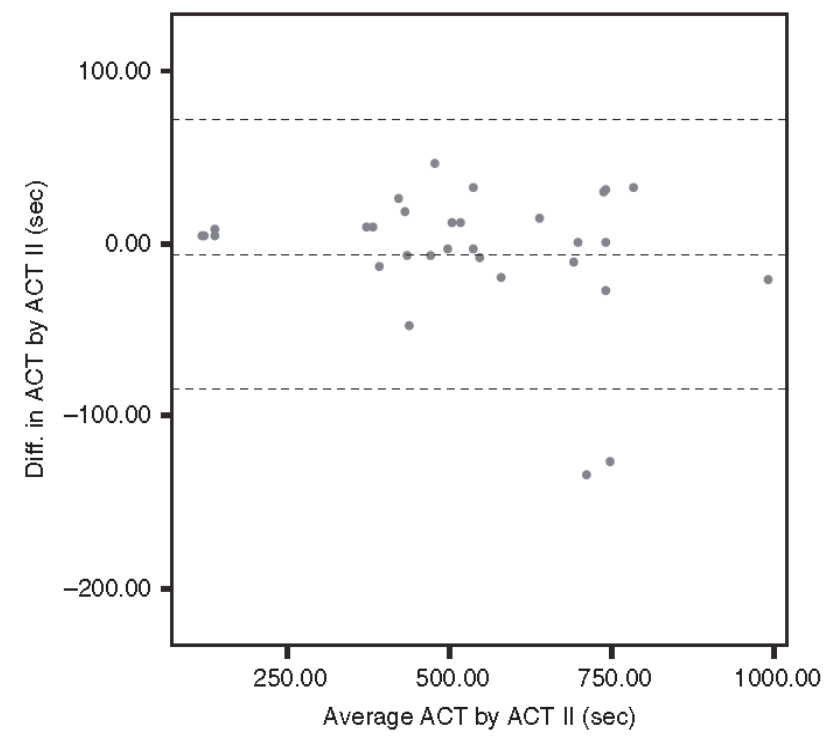

Figure 3: Repeatability of ACT Measurements by ACT II. 


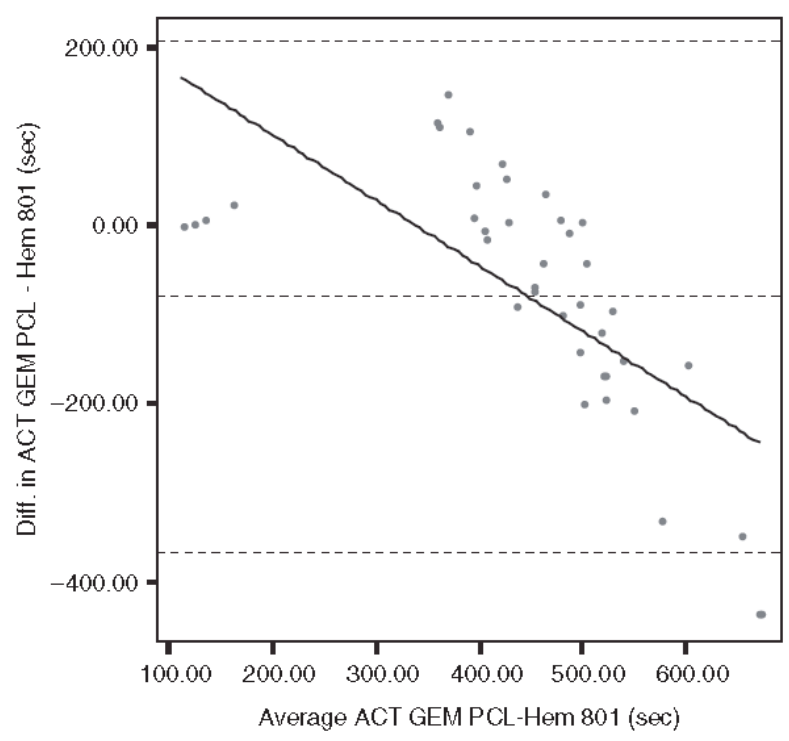

Figure 4: Agreement of ACT measurements between GEM PCL and Hemochron 801.

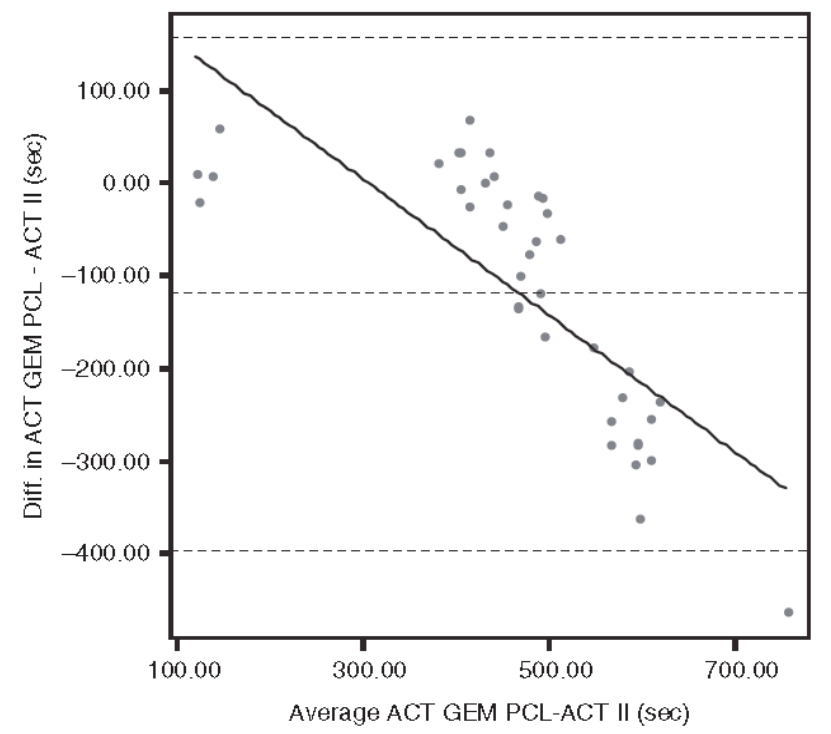

Figure 5: Agreement of ACT measurements between GEM PCL and ACT II. 


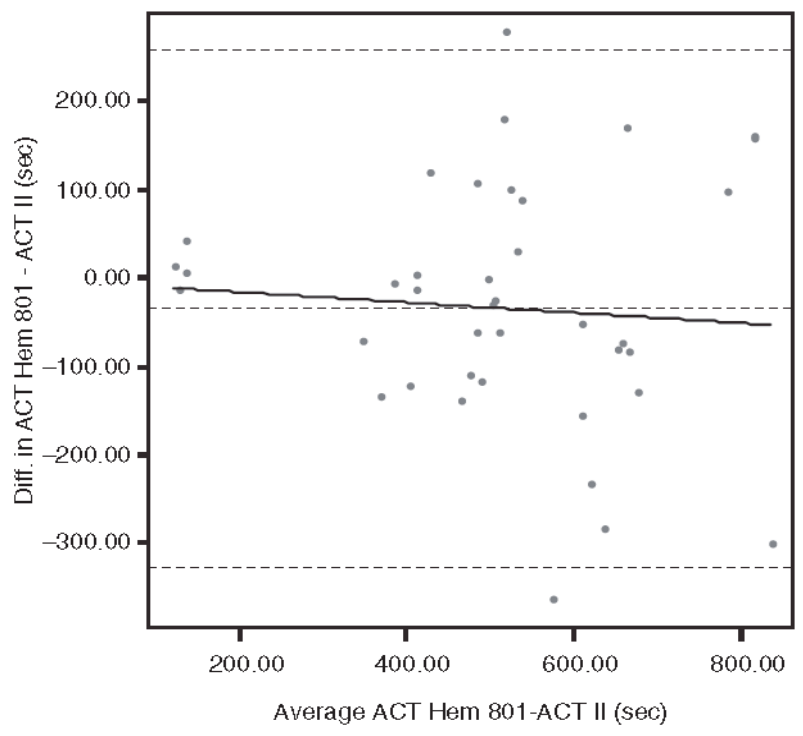

Figure 6: Agreement of ACT measurements between Hemochron 801 and ACT II.

Repeatability of the Hemochron 801 was measured by Zucker et al. as well, who used the variation coefficient to test repeatability [3].

In this study, the agreement between the analyzers was also investigated. This test demonstrated significant differences between the ACT values of the GEM PCL and both the Hemochron 801 and the ACT II. The Pearson correlation coefficient shows a rather strong negative correlation between the difference and the mean ACT for the GEM PCL compared with the Hemochron 801 ( $r=0.68$ ) and the ACT II ( $r=0.76)$. The ACT values yielded by the GEM PCL are clustered around $500 \mathrm{sec}$ (Figure 1), whereas the Hemochron 801 and the ACT II had a much higher spreading of the measured values in a range of 400-1000 sec (Figures 2 and 3). One possible explanation for this fact is that the Hemochron 801 and the ACT II both have a mechanical detection system, whereas the GEM PCL uses an optical detection system that could lead to another range of measurements. There could also be another possible explanation: it is known that the ACT may be prolonged by factors other than heparin, such as hemodilution and hypothermia, and, therefore, may not accurately reflect the extent of the anticoagulation by heparin [4]. Less sensitivity to these factors by the GEM PCL could be a possible cause of such differences in the spreading of the ACT. This finding should be evaluated in future studies.

In this study, hemodilution and hypothermia were not taken into consideration. There is sufficient evidence that a decreasing hematocrit during CPB increases the ACT [5-7]. Ignoring the hematocrit, it is not obvious to what degree the ACT differences between the analyzers can be explained by the analyzer itself and to what degree by 


\section{CHAPTER 4}

hemodilution. In total, 40 samples were taken. Unfortunately, there are five missing values for the GEM PCL, one missing value for the Hemochron 801 and nine missing values for the ACT II. These are all due to an insufficient or too large sample volume, not resuspending the activator in the right way or not taking sufficient time to warm up the cartridges.

\section{Conclusion}

All analyzers meet an acceptable level of repeatability. ACT values measured with the Hemochron 801 and the ACT II do not differ significantly, but do not agree with the ACT values of the GEM PCL. ACT measurements also depend on the analyzer that is used. Precautions should be taken in regard to generally accepted values because, among other factors, the measured values also seem to depend on the method of measurement. 


\section{References}

1. Hattersley PG. Activated coagulation time of whole blood. JAMA 1966; 196: 436- 40.

2. Bland JM, Altman DG. Statistical methods for assessing agreement between two methods of clinical measurement. Lancet 1986; 8: 307-10.

3. Zucker ML, Jobes C, Siegel M, Jobes D, LaDuca FM. Activated clotting time (ACT) testing: analysis of reproducibility. J Extra-Corporal Technol 1999; 31: 130-34.

4. Despotis GJ, Joist JH. Anticoagulation and anticoagulation reversal with cardiac surgery involving cardiopulmonary bypass: an update. J Cardiothorac Vasc Anesth 1999; 13: 18-29.

5. Despotis GJ, Summerfield AL, Joist JH et al. Comparison of activated coagulation time and whole blood heparin measurements with laboratory plasma anti-Xa heparin concentration in patients having cardiac operations. J Thorac Cardiovasc Surg 1994; 108: 1076-82.

6. Kmiecik SA, Liu JL, Vaadia TS et al. Quantitative evaluation of hypothermia, hyperthermia, and hemodilution on coagulation. J Extra-Corporal Technol 2001; 33: 100-105.

7. Niles SD, Sutton RG, Ploessl J, Pennell B. Correlation of ACT as measured with three commercially available devices with circulating heparin level during cardiac surgery. J Extra- Corporal Technol 1995; 27: $197-200$ 



\title{
CHAPTER 5
}

MEASUREMENTS OF THROMBIN

GENERATION INTRA-OPERATIVELY AND

ITS ASSOCIATION WITH BLEEDING

TENDENCY AFTER CARDIAC SURGERY

\author{
Yvonne P.J. Bosch \\ Raed Al Dieri \\ Hugo ten Cate \\ Patty J. Nelemans \\ Saartje Bloemen \\ Bas de Laat \\ Coenraad Hemker \\ Patrick W. Weerwind \\ Jos G. Maessen \\ Baheramsjah Mochtar
}

Based on:

Measurements of thrombin generation intra-operatively and its bleeding tendency after cardiac surgery. Thrombosis Research 133 (2014) 488-494. 


\section{Abstract}

Introduction: Patients undergoing cardiac surgery with cardiopulmonary bypass (CPB) are susceptible to hemostatic disturbances. Monitoring the hemostatic capacity by conventional clotting tests is challenging.

Materials and Methods: Thrombin generation (TG) by Calibrated Automated Thrombography, clotting tests and tissue factor pathway inhibitor (TFPI) measurements were performed to describe the relationship between hemostatic changes and alterations in these tests. Blood samples were collected before, during and after CPB. Furthermore, it was investigated whether TG measured intraoperatively, is associated with increased risk of bleeding postoperatively.

Results: TG diminished significantly $(\mathrm{p}<0.01)$ after heparinization in the presence and absence of platelets ( $37 \%$ and $50 \%$ ) compared to baseline. After the start of CPB, TG elevated and persisted till the end of surgery but remained lower than preoperatively. Activated clotting time increased after heparinization and after the start of bypass compared to baseline (400\% and $500 \%$ ). Anti-FXa activity reduced on the start of CPB compared to the level after heparinization, to almost the baseline value following protamine reversal of heparin. The plasma levels of total and free TFPI elevated 9 and 14 fold during bypass and remained after protamine administration higher than preoperatively. Plasma D-dimer levels reduced $(p<0.01)$ when bypass started. However, a marked elevation was observed in the following time points. TG in platelet-rich plasma measured after heparinization and after the start of CPB was associated $(p<0.05)$ with postoperative blood loss.

Conclusions: TG can be determined during CPB despite the high heparinization level, it reflects the hemostatic capacity better than clotting-based assays and might better predict bleeding when performed intraoperatively.

Keywords: cardiopulmonary bypass, heparinization, monitoring of hemostasis, thrombin generation, blood loss 


\section{Introduction}

Patients undergoing cardiac surgery with cardiopulmonary bypass (CPB) are susceptible to disturbances in the hemostatic system related to use of extracorporeal circulation. It is well known that despite of high doses of heparin sufficient thrombin is generated [1, 2]. Hemodilution causes reduction in plasma concentration of coagulation factors and platelet counts to decrease to about $50 \%$ of preoperative levels [3]. Hypothermia is related to both plasma coagulation factors and platelet dysfunction [4]. Consumption is caused by tissue injury, contact activation with the artificial surfaces of the extracorporeal circuit and the transfusion of shed pericardial blood [5]. Activation of both intrinsic and extrinsic pathways results in excessive thrombin generation and fibrinolysis [6]. High doses of unfractionated heparin are administered to create sufficient anticoagulation during $\mathrm{CPB}$, and are routinely neutralized by the administration of protamine sulfate. Heparins exert their anticoagulant activity by accelerating the inhibitory action of antithrombin (AT) on factor Xa and thrombin. Besides this, heparin augments the regulation of tissue factor dependent coagulation by releasing tissue factor pathway inhibitor (TFPI).

All of these aspects are causing defects in the coagulation system, and therefore, it is challenging to monitor the hemostatic capacity of a cardiac surgical patient in a proper way. The utility of the conventional coagulation tests for this purpose is poor because 1 ) these clotting tests are performed in platelet-poor citrated plasma, excluding the interaction between platelets with (pro- and anti-) coagulation factors; 2 ) the tests terminate with endpoints that occur when less than $5 \%$ of thrombin is formed [7], and 3) they show a weak relationship with heparin concentrations [8]. Moreover, these tests are poor predictors of perioperative bleeding [9], and a thrombotic tendency is not reflected in a shortened clotting time [10].

Because of the lack of a standard for measuring hemostatic capacity and the limitations of the conventional hemostatic measurements there is a need for tests that reflect better the hemostatic capacity of the patient's plasma [11]. Measuring thrombin generation, by calibrated automated thrombography (CAT), could be such a test. It reflects much, if not all, of the overall function of the blood clotting system [12]. All studies that used TG thus far showed high TG in all known prothrombotic conditions and predisposes for (re-)thrombosis [13-15]. TG is low in hemophiliac patients and restored by substitution therapy $[13,15]$. Moreover, recent clinical studies have demonstrated that the CAT is able to identify patients who are at an increased risk of bleeding after cardiac surgery $[9,16]$.

In this study we measured thrombin generation by CAT and conventional clotting tests in patients who underwent cardiac surgery with $\mathrm{CPB}$, in an attempt to describe the relationship between the changes in hemostatic equilibrium during surgery and 
the changes in these tests. In addition, we investigated whether thrombin generation measured intraoperatively when patients are already heparinized, is associated with an increased risk of bleeding after surgery.

\section{Materials and Methods}

\section{Study population}

The study was approved by the local ethics committee, the procedures followed were in accordance with the institutional and national ethical standards and with the Helsinki Declaration, and written informed consent was obtained from participating patients. In total, 30 male patients undergoing elective first-time coronary artery bypass grafting (CABG) were enrolled. Exclusion criteria were age $<18$ years, use of preoperative anticoagulation and platelet antagonists (excepting aspirin) within the preceding 5 days, known coagulopathy, impaired renal function, liver diseases resulting in elevated liver function tests and redo surgery.

\section{Clinical management}

General anaesthesia was induced using weight related dosing of sufentanil and etomidate, and muscle relaxation was achieved with pancuronium bromide. General anaesthesia was maintained during surgery using propofol. An initial dose of $300 \mathrm{IU} / \mathrm{kg}$ of body weight of heparin (Heparin Leo, Leo Pharmaceutical Products BV, Weesp, the Netherlands) was injected into a central venous line before initiation of CPB. The kaolin activated clotting time (ACT) was measured and, if the value was $\geq 400 \mathrm{~s}, C P B$ was initiated. If necessary, additional heparin was added. At the end of CPB, heparin was reversed by protamine chloride (Valeant Pharmaceuticals, Eschborn, Germany) at a 1:1 ratio of the loading dose.

All components of the CPB system were poly-2-methoxyethylacrylate coated (Terumo). The priming of the CPB circuit included 1,300 mL of $4 \%$ gelofusin, $200 \mathrm{~mL}$ $20 \%$ mannitol, $100 \mathrm{~mL}$ 20\% human albumin, $50 \mathrm{~mL} \mathrm{8.4 \%} \mathrm{NaHCO}_{3}$. and 6,500 IU unfractionated heparin (UFH). Retrograde autologous priming was used in most of the cases to reduce the priming volume by $200-500 \mathrm{~mL}$, resulting in less hemodilution. Normothermic perfusion $\left(36^{\circ} \mathrm{C}\right)$ was used during $\mathrm{CPB}$. Pericardial, pleural and residual blood of the CPB circuit after termination of CPB was drained and washed with a cell saver device. The transfusion trigger during CPB was set at a hematocrit below $23 \%$. 
Study design

Preoperative data collection included demographics and aspirin use preoperatively. The following variables were perioperatively registered: time on bypass, aortic clamp time, medication, infusion volumes, transfusion requirements, amount of packed red cells processed with the cell saver. Postoperatively, blood loss, determined by chest tube drainage after closing the chest, was measured until 20 hours after surgery.

Blood samples were collected at six time points: T1) pre-bypass before heparin administration, T2) pre-bypass after heparin administration, T3) during CPB, after placing the aortic clamp and cardioplegia administration, T4) 30 minutes after the start of $\mathrm{CPB}, \mathrm{T5}$ ) end of $\mathrm{CPB}, \mathrm{T6}) 5 \mathrm{~min}$ after protamine administration. These time points are representative of changes in the hemostatic equilibrium and provide more information on the responses to such changes. Blood samples before (T1-T2) and after CPB (T6) were withdrawn from the arterial line, after discarding the first $10 \mathrm{~mL}$. The other samples (T3-T5) were drawn from heart lung machine.

\section{Thrombin generation by Calibrated Automated Thrombography}

Arterial blood samples were collected into trisodium citrate and analyzed with CAT as previously reported [17]. Polybrene (Janssen Chimica, Beerse, Belgium) was used to neutralize heparinized samples, in order to explore the underlying thrombin generating capacity after heparinization and during CPB (T2-T5). Polybrene was used at 0.03 $\mathrm{mg} / \mathrm{mL}$ (final concentration) to fully neutralize the effect of UFH. The neutralizing effect of polybrene was investigated at varying heparin concentrations in normal pooled plasma (data not shown). TG was measured both in platelet-rich plasma (PRP) and in platelet-poor plasma (PPP). TG in PRP was performed within $1 \mathrm{~h}$ after blood withdrawal. PPP was stored at $-80^{\circ} \mathrm{C}$ until further analysis. The CAT assay was measured in a prewarmed plate fluorometer (Ascent reader, Thermolabsystems OY, Helsinki, Finland). In the PPP measurements, $10 \mu \mathrm{l}$ of tissue factor (Innovin, Dade-Behring, Marburg, Germany) and phospholipids (Avanti Polar Lipids Inc. Alabaster, AL, USA) in Hepes buffer containing $5 \mathrm{~g} / \mathrm{ml}$ bovine serum albumin (BSA) were added to each well, together with $10 \mu \mathrm{l}$ of buffer with or without polybrene. The final concentrations of tissue factor and phospholipids were $5 \mathrm{pM}$ and $4 \mu \mathrm{M}$, respectively. For PRP, the final concentration of tissue factor was $1 \mathrm{pM}$. Thrombin generation was initiated by adding $20 \mu \mathrm{l}$ of Z-Gly-Gly-Arg-aminomethylcoumarine (2.5 mM) (Bachem, Basel, Switzerland) and $\mathrm{CaCl} 2$ (100 mM) in Hepes buffer containing $60 \mathrm{mg} / \mathrm{ml} \mathrm{BSA}$ ). Data were analyzed using ThrombinoscopeTM software (Thrombinoscope bv, Maastricht, the Netherlands). CAT parameters which are used to determine correlation of TG with postoperative blood loss are: 1) lag time (min): the initiation phase of clotting and equals the 
clotting time; 2 ) peak height ( $\mathrm{nM})$ : the maximal amount of thrombin formed; 3 ) endogenous thrombin potential (ETP) ( $\left.\mathrm{nM}^{*} \mathrm{~min}\right)$ : the area under the TG curve; and 4) time to peak ( $\mathrm{min}$ ): the time needed to achieve the peak height. The general form of the thrombogram in the different conditions is shown in Figure 1.

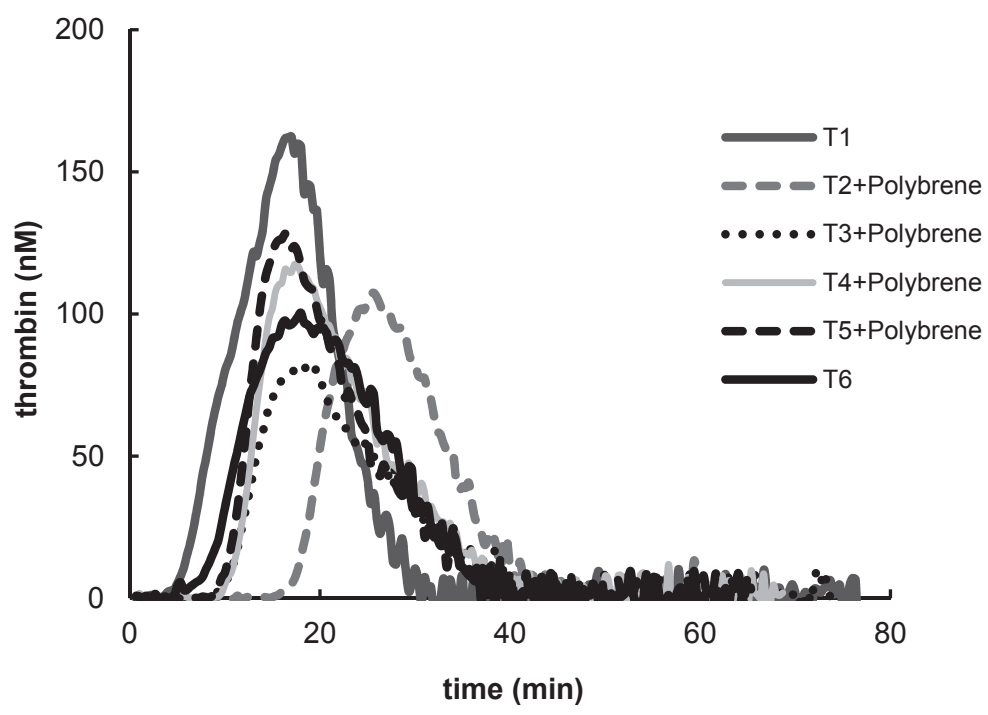

Figure 1 Representative figure of the calibrated automated thrombography in platelet-rich plasma before and during surgery. T1) pre-bypass before heparin administration, T2) pre-bypass after heparin administration, T3) during $\mathrm{CPB}$, after placing the aortic clamp and cardioplegia administration, T4) 30 minutes after the start of $\mathrm{CPB}, \mathrm{T5}$ ) end of $\mathrm{CPB}, \mathrm{T} 6) 5 \mathrm{~min}$ after protamine administration.

\section{Routine laboratory tests and TFPI measurement}

Besides CAT measurements, ACT (kaolin-activated, Hemotec ACT II Automated Coagulation Timer, Medtronic, Inc. Minneapolis, MN, USA) and laboratory parameters hematocrit, hemoglobin, thrombocytes (Coulter LH750 hematology analyzer, Beckman Coulter Inc. Miami, USA), fibrinogen, antithrombin (Siemens Berichrom Antithrombin III kit, Marburg Germany), D-dimers, prothrombin time (PT) and activated partial thromboplastin time (aPTT) were determined to assess patient's hemostatic profile. Anti-factor $\mathrm{Xa}(\mathrm{aXa})$ was also measured in all samples, using a competition method without addi- 
tion of exogenous antithrombin (Biophen Heparin (LRT), Hyphen Biomed, Neuville sur Oise, France).

Plasma level of free and total TFPI was determined by use of ELISA (Asserachrom ${ }^{\circledR}$ free TFPI and Asserachrom ${ }^{\circledR}$ total TFPI, Diagnostica Stago, Asnières, France). Assays were performed according to manufacturer's instructions.

\section{Statistics}

Statistical analysis was performed with SPSS for Windows 20.0 (SPSS, Inc., Chicago, IL, USA). Results are expressed as mean and standard deviation or standard error of the mean. Measurements on T3-T5 were compared with measurements on T2 by Wilcoxon signed rank test for related samples.

Patients were divided into two groups with high versus low blood loss, where the median value of blood loss volume at 20 hours postoperatively was used as cut-off value. Both groups were compared with respect to mean values of CAT parameters with the Mann-Whitney $U$ test for independent samples. In addition Spearman correlation was calculated in order to show the association between blood loss as a continuous variable and the thrombin generation parameters.

The ability of CAT to discriminate between the two groups was also evaluated by the construction of a receiver operating characteristic (ROC) curve. The corresponding area under the curve (AUC) with $95 \%$ confidence interval was used to quantify the predictive value of the parameters.

\section{Results}

Thirty male patients were enrolled into the study. One patient was excluded because complicated adhesiolysis was necessary to achieve access to the heart resulting in excessive blood loss. Age, preoperative use of aspirin, and intraoperative data relating to the 29 patients are shown in Table 1.

Heparin and protamine administration: effects on anti-Xa and TFPI

The level of anti-factor Xa activity reduced significantly $(p<0.001)$ on the start of CPB (4.2 $\pm 0.6 \mathrm{U} / \mathrm{ml}$ at T3) compared to the plasma level following unfractionated heparin (UFH) administration $(6.7 \pm 1.1 \mathrm{U} / \mathrm{ml}$ at $\mathrm{T} 2)$ and remained unchanged until the end of CPB (Table 2). However, a decrease was observed following protamine administration $(0.3 \pm 0.6 \mathrm{U} / \mathrm{ml})$.

Total TFPI plasma levels elevated about 8.9-fold during CPB (Figure 2) and returned to levels, which are slightly higher than baseline following protamine admin- 
istration. However, free TFPI increased 13.8-fold after heparinization and decreased to levels that remained above baseline after protamine reversal of heparin (Figure 2).

Table 1: Distribution of patient characteristics and intra-operative variables.

\begin{tabular}{|c|c|}
\hline & $N=29$ \\
\hline \multicolumn{2}{|l|}{ Patient characteristics } \\
\hline Age (years) & $65 \pm 10$ \\
\hline Weight (kg) & $86 \pm 11$ \\
\hline Hypertension $n$ (\%) & $22(75.9 \%)$ \\
\hline Hypercholesterolemia $n(\%)$ & $22(75.9 \%)$ \\
\hline Diabetes $n(\%)$ & $8(27.6 \%)$ \\
\hline Stroke $n(\%)$ & $2(6.9 \%)$ \\
\hline Peripheral vessel disease $n(\%)$ & $6(20.7 \%)$ \\
\hline Preoperative creatinine ( $\mu \mathrm{mol} / \mathrm{L})$ & $88 \pm 14$ \\
\hline Aspirin exp. preop. $n(\%)$ & $27(93.1 \%)$ \\
\hline Total log. EUROscore & $2.3 \pm 1.4$ \\
\hline \multicolumn{2}{|l|}{ Intraoperative variables } \\
\hline Tranexamic acid $n(\%)$ & $14(48.3 \%)$ \\
\hline Heparin-total (IU/kg) & $336 \pm 54$ \\
\hline Protamine-total (mg/kg) & $3.35 \pm 0.52$ \\
\hline Crystalloids (ml/kg) & $16.9 \pm 6.5$ \\
\hline Colloid $(\mathrm{ml} / \mathrm{kg})^{*}$ & $3.9(0-16.3)$ \\
\hline PRC (units)* & $0.0(0.0-1.0)$ \\
\hline FFP (units) & $0 \pm 0$ \\
\hline PLT (units)* & $0.0(0.0-1.0)$ \\
\hline PRC cell saver $(\mathrm{ml} / \mathrm{kg})$ & $5.7 \pm 2.0$ \\
\hline Time on bypass (min) & $76 \pm 20$ \\
\hline Time X-clamp (min) & $48 \pm 16$ \\
\hline Nr. of arterial grafts & $0.9 \pm 0.4$ \\
\hline Nr. of venous grafts & $2.5 \pm 1.1$ \\
\hline
\end{tabular}

Data shown are mean \pm SD or number (\%). FFP = fresh frozen plasma; PLT = platelets; $\mathrm{PRC}=$ packed red cells. 
The level of anti-factor Xa activity reduced significantly $(p<0.001)$ at the start of CPB (4.2 $\pm 0.6 \mathrm{U} / \mathrm{ml}$ at T3) compared to the plasma level following unfractionated heparin (UFH) administration $(6.7 \pm 1.1 \mathrm{U} / \mathrm{ml}$ at T2) and remained unchanged until the end of CPB (Table 2). However, a decrease was observed following protamine administration $(0.3 \pm 0.6 \mathrm{U} / \mathrm{ml})$.

Total TFPI plasma levels elevated about 8.9-fold during CPB (Figure 2) and returned to levels, which are slightly higher than baseline following protamine administration. However, free TFPI increased 13.8-fold after heparinization and decreased to levels that remained above baseline after protamine reversal of heparin (Figure 2).

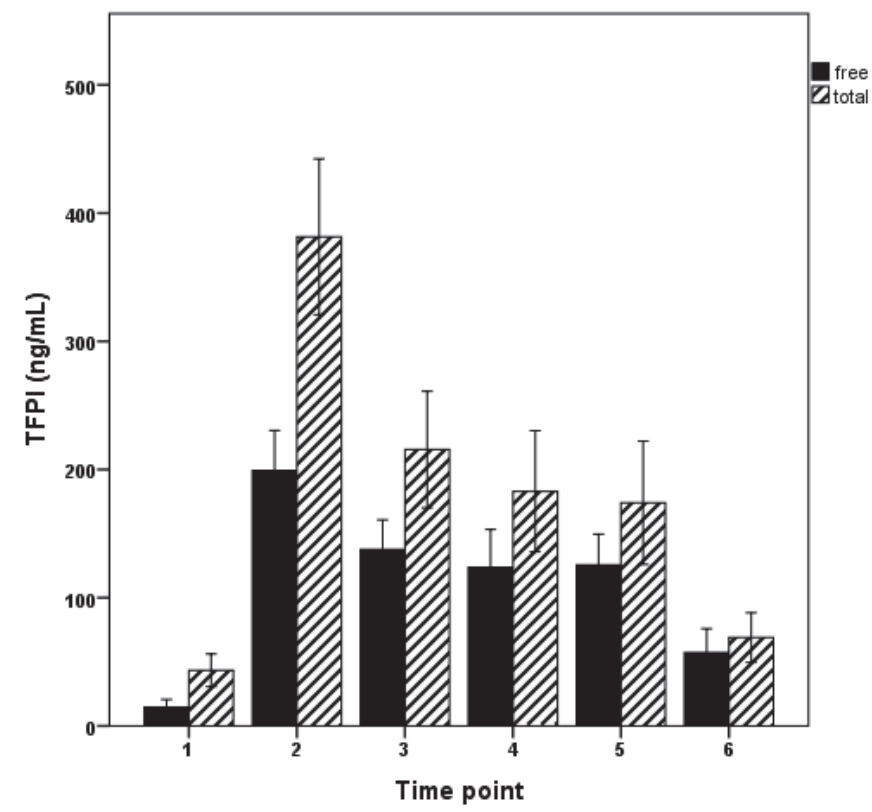

Figure 2: Plasma levels of free and total tissue factor pathway inhibitor (TFPI) before, during and after cardiopulmonary bypass. Data are presented as mean \pm SD. T1) baseline, T2) pre-bypass after heparin administration, T3) during $\mathrm{CPB}$, after placing the aortic clamp and cardioplegia administration, $\mathrm{T4}$ ) 30 minutes after the start of $(P B, T 5)$ end of $(P B, T 6) 5$ min after protamine administration.

Hemodilution during $C P B$

Hemodilution during cardiac surgery is a standard practice as it reduces the need for intraoperative and postoperative blood transfusion and other related risks. Hemato- 
crit, hemoglobin and platelet count were determined to assess the degree of hemodilution. Table 2 shows that maximal hemodilution was achieved after cardioplegia administration, 5-10 minutes after the start of CPB (T3), and the level of the above parameters decreased significantly $(p<0.001)$ compared with T2 (platelet count at T2 was $215 \pm 60\left(* 1000 / \mathrm{mm}^{3}\right)$, and at T3: $\left.149 \pm 48\left(* 1000 / \mathrm{mm}^{3}\right)\right)$, and remained below this level until the end of the procedure $(p<0.001)$.

\section{Fibrinogen and antithrombin}

A significant reduction in the plasma levels of fibrinogen and antithrombin $(p<0.001)$ were also observed shortly after the start of CPB and remained unchanged after protamine reversal of heparin $(p<0.001)$, Table 2 .

Table 2: Changes of the routine laboratory tests before and during surgery

\begin{tabular}{|c|c|c|c|c|c|c|}
\hline & $\mathrm{T} 1$ & $\mathrm{~T} 2$ & T3 & T4 & T5 & T6 \\
\hline $\mathrm{ACT}$ (sec) & $132 \pm 15$ & $533 \pm 135$ & $656 \pm 135 * * *$ & $567 \pm 110^{*}$ & $530 \pm 91$ & $140 \pm 17$ \\
\hline aPTT (sec) & $28.9 \pm 3.2$ & & & & & $34.6 \pm 5.5$ \\
\hline PT (sec) & $11.5 \pm 0.5$ & & & & & $15.4 \pm 1.5$ \\
\hline Hemoglobin (mmol/L) & $7.8 \pm 0.6$ & $7.7 \pm 0.6$ & $5.0 \pm 0.5^{* * *}$ & $5.3 \pm 0.5^{* * *}$ & $5.4 \pm 0.4^{* * *}$ & $5.4 \pm 0.5$ \\
\hline Hematocrit (\%) & $37 \pm 3$ & $37 \pm 3$ & $24 \pm 3^{* * *}$ & $25 \pm 2 * * *$ & $26 \pm 2 * * *$ & $26 \pm 2$ \\
\hline Platelet count $\left(x 1000 / \mathrm{mm}^{3}\right)$ & $215 \pm 61$ & $215 \pm 60$ & $149 \pm 48 * * *$ & $173 \pm 54 * * *$ & $176 \pm 54 * * *$ & $128 \pm 42$ \\
\hline Fibrinogen (g/L) & $2.9 \pm 0.9$ & $2.7 \pm 0.6$ & $1.7 \pm 0.4^{* * *}$ & $1.7 \pm 0.4^{* * *}$ & $1.8 \pm 0.4^{* * *}$ & $1.7 \pm 0.4$ \\
\hline Antithrombin (\%) & $81 \pm 11$ & $83 \pm 9$ & $48 \pm 6 * * *$ & $49 \pm 8^{* * *}$ & $50 \pm 8^{* * *}$ & $50 \pm 7$ \\
\hline D-dimers (ng/mL) & $700 \pm 782$ & $693 \pm 808$ & $547 \pm 716^{* * *}$ & $776 \pm 899$ & $912 \pm 1018^{*}$ & $1109 \pm 1146$ \\
\hline Anti-Xa (U/mL) & \pm 0.0 & $6.7 \pm 1.1$ & $4.2 \pm 0.6 * * *$ & $4.2 \pm 0.6 * * *$ & $4.1 \pm 0.7 * * *$ & $0.3 \pm 0.6$ \\
\hline
\end{tabular}

Data are shown as mean \pm SD. ACT = activated clotting time; aPTT = activated partial thromboplastin time; PT = prothrombin time; ${ }^{*}=p<0.05$ vs. T2; ${ }^{* *}=p<0.01$ vs. T2; ${ }^{* *}=p<0.001$ vs. T2. T1) pre-bypass before heparin administration, T2) pre-bypass after heparin administration, T3) during CPB, after placing the aortic clamp and cardioplegia administration, T4) 30 minutes after the start of CPB, T5) end of CPB, T6) 5 min after protamine administration.

\section{Effect of hemodilution on D-dimer levels and thrombin generation}

Coagulation in vivo was assessed by measuring the D-dimer level before, during and after CPB. Table 2 and Figure 3 show that the level of D-dimers reduced significantly $(p<0.001)$ when CPB starts (693 $\pm 808 \mathrm{ng} / \mathrm{ml}$ at T2; $547 \pm 716 \mathrm{ng} / \mathrm{mL}$ at T3). However, a marked elevation was seen in the following time points with an increase of about 1.5fold after protamine administration $(T 6, p<0.001)$. 
Calibrated thrombin generation was determined ex vivo in platelet-rich and platelet-poor plasma before, during and after CPB. Heparinized samples (T2, T3, T4, T5) were neutralized with polybrene in order to investigate the underlying thrombin generating capacity. Table 3 shows that the main parameters of the thrombogram (ETP and peak thrombin) diminished considerable following heparinization (ETP in PRP: $1190 \pm 418$ nM*min; Peak in PRP: $82 \pm 38 \mathrm{nM}$ ) compared to baseline (ETP in PRP: 1881 $\pm 359 \mathrm{nM} *$ min; Peak in PRP: $133 \pm 42 \mathrm{nM}$ ). ETP elevated during CPB, to reach a level that was

Table 3: Parameters of the thrombin generation test before and during surgery.

\begin{tabular}{|c|c|c|c|c|c|c|}
\hline & $\mathrm{T} 1$ & $\mathrm{~T} 2$ & T3 & $\mathrm{T} 4$ & T5 & T6 \\
\hline \multicolumn{7}{|l|}{ PRP: } \\
\hline Lag time (min) & $8.7 \pm 3.0$ & $20.6 \pm 6.4$ & $12.2 \pm 2.3^{* * *}$ & $11.2 \pm 4.3^{* * *}$ & $10.4 \pm 2.1 * * *$ & $8.7 \pm 3.2$ \\
\hline $\operatorname{ETP}\left(\mathrm{nM}^{*} \min \right)$ & $1881 \pm 359$ & $1190 \pm 418$ & $1266 \pm 365$ & $1491 \pm 318^{* * *}$ & $1511 \pm 259 * *$ & $1524 \pm 264$ \\
\hline Peak (nM) & $133 \pm 42$ & $82 \pm 38$ & $73 \pm 22$ & $95 \pm 23$ & $96 \pm 25$ & $73 \pm 19$ \\
\hline Time to peak ( $\mathrm{min}$ ) & $19.3 \pm 4.8$ & $29.4 \pm 7.9$ & $20.7 \pm 3.9 * * *$ & $18.8 \pm 6.1^{* * *}$ & $18.2 \pm 3.3^{* * *}$ & $20.3 \pm 5.6$ \\
\hline \multicolumn{7}{|l|}{ PPP: } \\
\hline Lag time (min) & $2.5 \pm 0.5$ & $10.2 \pm 3.2$ & $5.2 \pm 1.2 * * *$ & $5.0 \pm 1.0 * * *$ & $5.1 \pm 1.0 * * *$ & $4.2 \pm 0.9$ \\
\hline $\operatorname{ETP}\left(\mathrm{nM}^{*} \min \right)$ & $1587 \pm 355$ & $806 \pm 437$ & $1119 \pm 403^{* *}$ & $1068 \pm 325^{* *}$ & $1066 \pm 319 * *$ & $1508 \pm 297$ \\
\hline Peak (nM) & $321 \pm 63$ & $97 \pm 72$ & $83 \pm 39$ & $91 \pm 41$ & $96 \pm 46$ & $241 \pm 36$ \\
\hline Time to peak ( $\mathrm{min}$ ) & $4.7 \pm 0.7$ & $14.6 \pm 3.8$ & $10.1 \pm 1.9^{* * *}$ & $9.5 \pm 1.7^{* * *}$ & $9.5 \pm 1.7^{* * *}$ & $6.7 \pm 1.1$ \\
\hline
\end{tabular}

Data are shown as mean \pm SD. PRP = platelet rich plasma; $\mathrm{PPP}=$ platelet poor plasma; $\mathrm{ETP}=$ endogenous thrombin potential; ${ }^{*}=\mathrm{p}<0.05$ vs T2; ${ }^{* *}=\mathrm{p}<0.01$ vs T2; ${ }^{* *}=\mathrm{p}<0.001$ vs T2. T1) pre-bypass before heparin administration, T2) pre-bypass after heparin administration, T3) during CPB, after placing the aortic clamp and cardioplegia administration, T4) 30 minutes after the start of CPB, T5) end of CPB, T6) 5 min after protamine administration.

significantly higher than the level after heparinization $(p<0.01)$. ETP levels at different time points are shown in Figure 3. The lag time (a clotting dependent variable) increased significantly after heparinization and during CPB $(p<0.001)$, but not after protamine administration (in PRP). Time to peak increased significantly $(p<0.001)$ during CPB and remained prolonged even after protamine administration in PPP. In PRP, however, time to peak only increased at $\mathrm{T} 2$.

\section{Clotting time-based tests}

Activated clotting time (ACT) was measured before, during and after CPB. ACT prolonged following heparinization (ACT baseline: $132 \pm 15 \mathrm{~s}$; ACT at T2: $533 \pm 135 \mathrm{~s}$ ) and 
increased further immediately after the start of CPB (656 $\pm 135 \mathrm{~s} ; \mathrm{p}<0.001)$. Then it shows a gradual decrease during CPB. The ACT values are summarized in Figure 3. After protamine administration, it decreased to a level slightly higher than baseline. Activated partial thromboplastin time (aPTT) and prothrombin time (PT) remained prolonged after heparin neutralization, see Table 2 .

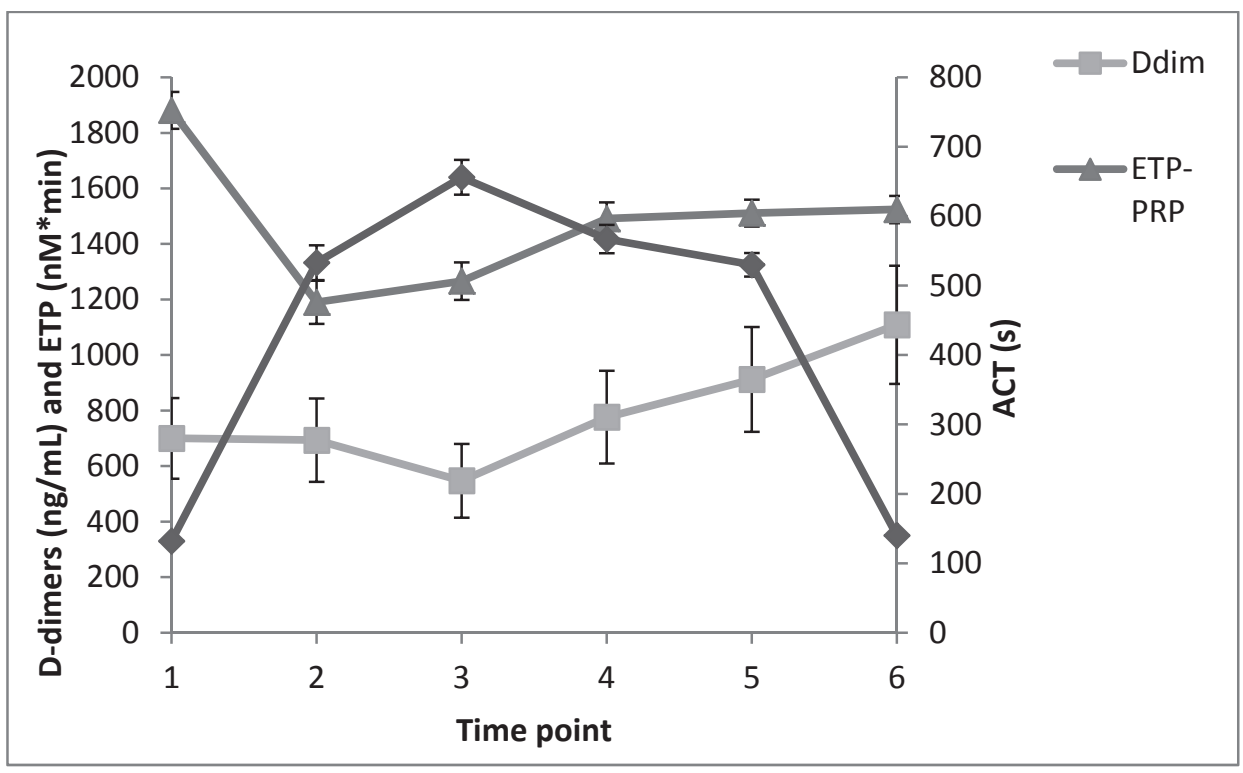

Figure 3: ETP in PRP, ACT and D-dimers before, during and after cardiopulmonary bypass.

Data are shown as mean \pm standard error of the mean. T1) baseline, T2) pre-bypass after heparin administration, T3) during CPB, after placing the aortic clamp and cardioplegia administration, T4) 30 minutes after the start of $\mathrm{CPB}, \mathrm{T5}$ ) end of $\mathrm{CPB}, \mathrm{T} 6) 5 \mathrm{~min}$ after protamine administration.

Association of ex vivo thrombin generation and other perioperative variables with postoperative bleeding

Patients were divided in two groups using the median volume of postoperative blood loss until 20 hours as a cut-off value (group 1 patients with median blood loss $<930 \mathrm{~mL}$ and group 2 for patients with median blood loss $\geq 930 \mathrm{~mL}$ ). The association of postoperative blood loss with the parameters of the thrombogram (peak, ETP, time to peak and lag time) in PRP measured intra-operatively (after heparinization and start of CPB) was investigated in the two groups (Table 4 and Figure 4). It was observed that peak height (for both T2 and T3) in group 2 is significantly lower than the one of group 1 
(Peak at T2 in group 1: $97 \pm 45 \mathrm{nM}$; Peak at T2 in group 2: $69 \pm 26 \mathrm{nM}$ ). Blood loss as a continuous variable correlated only significantly with peak height at T3 $(p=0.002)$. A receiver operating curve $(\mathrm{ROC})$ was used to assess which parameters predict bleeding after surgery. The area under the curve (AUC) of the ROC shows that the peak height in PRP at T2 (after heparinization) and at T3 (5-10 min after the start of CPB) associated significantly $(p<0.05)$ with blood loss postoperatively (AUC at T2: 0.738; AUC at T3:

Table 4: Thrombin generation parameters measured in PRP before (T2) and after CPB (T3) and their association with postoperative blood loss.

\begin{tabular}{|c|c|c|c|c|c|c|c|}
\hline & $\begin{array}{l}\text { Spearman } \\
\text { correlation }\end{array}$ & $p$-value & $\begin{array}{l}\text { Blood loss < } \\
930 \mathrm{~mL}\end{array}$ & $\begin{array}{l}\text { Blood loss } \geq \\
930 \mathrm{~mL}\end{array}$ & $\mathrm{p}$-value & $\begin{array}{l}\text { AUC } \\
(95 \% \mathrm{Cl})\end{array}$ & $p$-value \\
\hline \multicolumn{8}{|l|}{ T2 } \\
\hline Lag time (min) & 0.102 & 0.600 & $20.1 \pm 7.7$ & $21.2 \pm 5.0$ & 0.949 & $\begin{array}{l}0.510 \\
(0.292-0.727)\end{array}$ & 0.930 \\
\hline $\operatorname{ETP}\left(\mathrm{nM}^{*} \min \right)$ & -0.298 & 0.117 & $1298 \pm 524$ & $1090 \pm 269$ & 0.057 & $\begin{array}{l}0.710 \\
(0.503-0.916)\end{array}$ & 0.055 \\
\hline Peak (nM) & -0.330 & 0.081 & $97 \pm 45$ & $69 \pm 26$ & 0.029 & $\begin{array}{l}0.738 \\
(0.544-0.932)\end{array}$ & 0.029 \\
\hline Time to peak (min) & 0.304 & 0.109 & $28.1 \pm 10.0$ & $30.6 \pm 5.4$ & 0.747 & $\begin{array}{l}0.619 \\
(0.408-0.830)\end{array}$ & 0.743 \\
\hline \multicolumn{8}{|l|}{ T3 } \\
\hline Lag time (min) & 0.092 & 0.636 & $12.3 \pm 2.7$ & $12.0 \pm 1.8$ & 0.914 & $\begin{array}{l}0.543 \\
(0.322-0.764)\end{array}$ & 0.913 \\
\hline $\operatorname{ETP}\left(\mathrm{nM}^{*} \min \right)$ & -0.204 & 0.288 & $1379 \pm 401$ & $1161 \pm 304$ & 0.201 & $\begin{array}{l}0.643 \\
(0.436-0.850)\end{array}$ & 0.190 \\
\hline Peak (nM) & -0.546 & 0.002 & $84 \pm 27$ & $63 \pm 10$ & 0.006 & $\begin{array}{l}0.793 \\
(0.605-0.981)\end{array}$ & 0.007 \\
\hline Time to peak (min) & 0.058 & 0.764 & $20.9 \pm 4.9$ & $20.4 \pm 3.0$ & 0.914 & $\begin{array}{l}0.512 \\
(0.290-0.734)\end{array}$ & 0.913 \\
\hline
\end{tabular}

Association of thrombin generation parameters with blood loss, both as a continuous variable (by Spearman correlation) and as a dichotomous variable (by Students T-test). Data are shown as mean \pm SD. PRP = platelet rich plasma; ETP = endogenous thrombin potential; T2) pre-bypass after heparin administration, T3) during $\mathrm{CPB}$, after placing the aortic clamp and cardioplegia administration; $\mathrm{AUC}=$ area under the $\mathrm{curve} ; \mathrm{Cl}=$ confidence interval

0.793), and to a lesser extent was the ETP (AUC at T2: 0.710; AUC at T3: 0.643) (see Table 4). The time-dependent parameters did not show any predictive value for bleeding after surgery. However, thrombin generation in PPP was not significantly different between the two groups (data not shown). In addition, it was analyzed whether other perioperative variables differed significantly between the patients who bled more or less than $930 \mathrm{~mL}$ postoperatively. Platelet count was the only variable that was significantly different between the two groups (both at T2 and T3) (data not shown). 


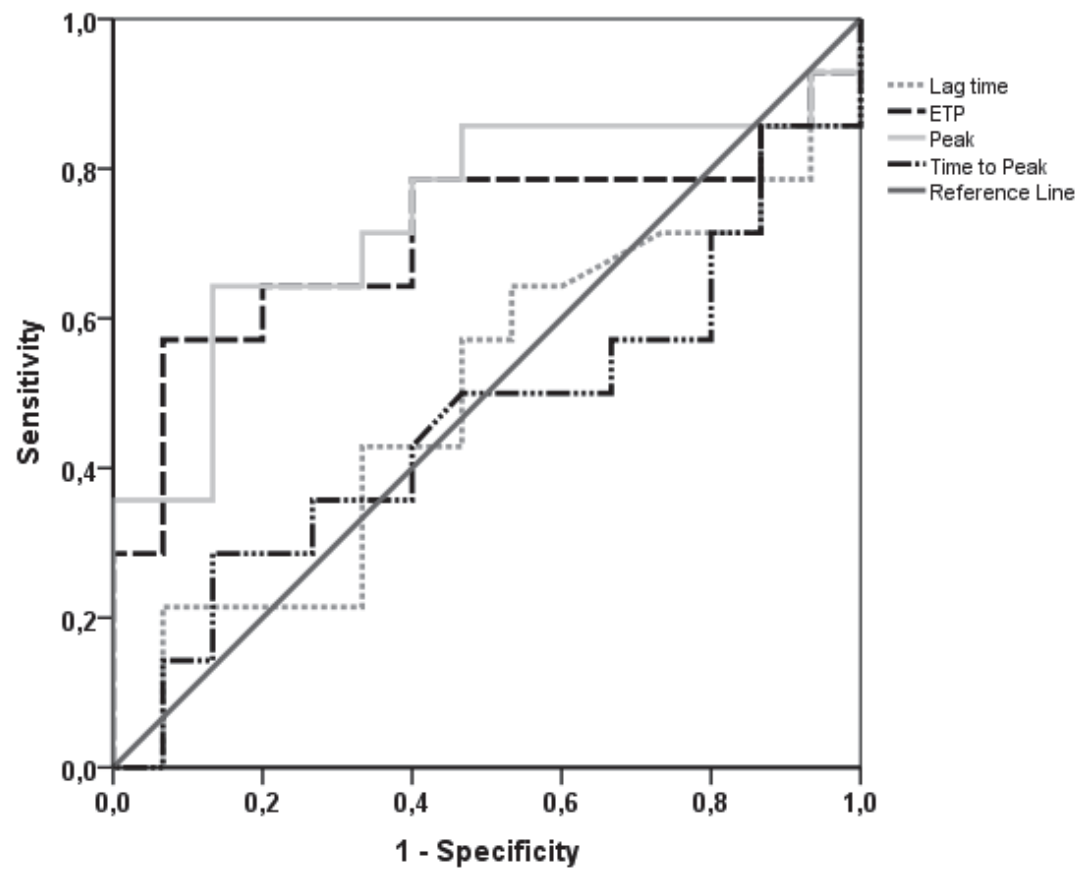

Figure 4: Receiver operating curve (ROC) for assessing the discriminative capacity of thrombin generation parameters in PRP after heparinization in predicting blood loss of more than $930 \mathrm{~mL}$.

\section{Discussion}

In the present study conventional clotting tests used during cardiac surgery with CPB and the thrombin generation (TG) assay were studied to investigate the relationship between the changes in hemostatic equilibrium during surgery and the changes in these tests to reveal the fundamental mechanisms leading to disturbed hemostasis. Besides this the association between the CAT assay during surgery and blood loss after CPB was investigated.

After the start of the cardiopulmonary bypass, the parameters of the thrombin generation test responded immediately. The mean levels of the ETP elevated again and both lag time and time to peak decreased, suggesting activation of the clotting system. In contrast, the mean levels of the D-dimers further decreased while the levels of ACT continued to increase particularly at $\mathrm{T} 3$, indicating that these parameters do not reflect the hypercoagulability induced by CPB. Hence, ex vivo measurements of thrombin generation were better associated with the hemostatic changes during CPB than the 
clotting-based assays and/or D-dimers measurement. It is well known that cardiac surgery with CPB results in an excessive activation of the coagulation system, despite of clinically adequate heparinization. This illustrates the magnitude of the procoagulant effect during CPB $[1,2]$. The hemostatic activation induced by CPB is related to the exposure of blood to the artificial surfaces of the extracorporeal circuit and to surgical trauma [18].

Anticoagulation with high doses of heparin in cardiac surgery diminishes TG [19, 20]. Previously it was demonstrated that TG is superior to the APTT in detecting the effect of low doses of heparin [21]. ETP was diminished completely after heparinization (T2) because of the high doses of heparin administered. In order to explore the underlying thrombin generating capacity during $\mathrm{CPB}$, heparinized samples (T2-T5) were neutralized by polybrene. After heparin neutralization, we observed a significant decrease in ETP and peak thrombin and a prolongation in lag time and time to peak (both in PRP and in PPP) on T2 compared to baseline. This could be due to the marked increase of the TFPI level (14 times higher at T2 compared to T1). When heparin is administered, TFPI is released from the endothelial cells [22]. TFPI is the only specific inhibitor of the TF-FVIla complex, regulating both its procoagulant and proinflammatory properties [23]. After the start of CPB, ETP elevated again and lag time and time to peak decreased. TFPI concentrations dropped on T3 which could again be the reason for accompanying changes in TG parameters. However, during CPB the ETP (in PRP, not in PPP) continued to increase, which cannot be explained by TFPI levels, as it remains stable at T4 and T5. In addition, hemodilution will only have a minor effect on tissue factor induced TG since dilution of plasma does not significantly affect TG when the concentrations of prothrombin and antithrombin are kept constant [24]. There is significant variability in the TFPI response to heparinization in patients undergoing CPB. Some patients do not respond to heparin with an increase in TFPI levels [25]. On average, according to our data, CPB with heparin anticoagulation causes a highly significant increase in both total and free TFPI levels. This is in agreement with previous studies $[26,27]$. When heparin is neutralized with protamine after CPB, TFPI levels decrease, but remain higher than preoperatively. These findings confirm previous reports as well $[26,28]$. The remaining TFPI may contribute to ongoing anticoagulation, as reflected in significant differences in TG parameters after protamine administration compared with baseline.

That thrombin is generated progressively during cardiac surgery with CPB despite of high doses of heparin, is also demonstrated by studies which show an increase in concentrations of thrombin-antithrombin complexes, prothrombin fragment 1.2 and fibrin degradation products (e.g. D-dimers), suggesting coagulation in vivo [2, 29, 30]. In this study the D-dimer level does not react on heparinization, and does not show activation of hypercoagulation immediately after the start of bypass. In contrast, D- 
dimers decreased on T3. Only after 30 minutes on bypass D-dimers start to rise. In addition, it is questionable if we can rely on the value of this in vivo test because of the large standard deviation it shows.

Our results show that heparin concentration measured by anti-factor Xa (aXa) was maximal on T2 (5 minutes after heparin administration), and decreased to a constant level after the start of CPB (T3-T5). On T6 heparin was neutralized by protamine, leading to minimal aXa levels. Various studies have reported clinically significant variability in the results of different aXa assays [31-33]. Anti-factor Xa measurement is not a reliable test for determining the concentration of antithrombin-binding heparin and its effect on the clotting system of the patient [34]. Inhibition of coagulation does not only depend on aXa activity but also on anti-thrombin activity (alla).

Activated clotting time (ACT) has become a widely accepted point-of-care test for monitoring heparin during CPB [35]. In this study an ACT of more than $400 \mathrm{~s}$ was considered to indicate adequate anticoagulation to establish CPB. The ACT after heparinization was prolonged from $132 \mathrm{~s}$ at baseline to $533 \mathrm{~s}$. After the start of CPB the ACT did not show any effect of hypercoagulation on T2: the ACT continued to increase, reaching its maximal value on T3, despite of an decreasing anti-Xa level. This confirms previous findings that ACT values correlate poorly with anti-Xa heparin concentrations [8, 36]. However, ACT may be prolonged by factors such as hypothermia, qualitative platelet abnormalities, and antifibrinolytic drugs [35].

The PT and aPTT could only be measured before heparinization and after protamine administration. Both the aPTT and the PT were significantly prolonged after heparin neutralization $(\mathrm{T} 6)(\mathrm{P}<0.001)$. Previous reports showed their poor predictive value for bleeding and re-thrombosis [37, 38].

From the above it is evident that clotting-based assays and anti-Xa are poor tools for assessing the coagulation system before and during cardiac surgery. Here we investigated whether thrombin generation would do better.

Thrombin generation was found to predict bleeding tendency when performed intraoperatively and particularly in the presence of platelets (Figure 4). When the area under the ROC curve was determined for the parameters of thrombin generation, it was clear that the overall performance of the peak height and ETP was much better than the lag time and time to peak and that they were also predictable for blood loss after surgery. Because the lag time for all purposes is equal to the clotting time, this is in line with the observation that the ACT has a poor predictive value. In our previous paper [16], baseline ACT failed to predict bleeding tendency after cardiac surgery. The additional value of the current data is that even intraoperatively, after heparinization, TG could be predictive for the risk of bleeding after cardiac surgery. Previous studies investigated only the predictive value of pre- and postoperative thrombin generation and thromboelastometry for blood loss after cardiac surgery [9, 16, 39]. These studies 
found that a lower capacity for thrombin generation in plasma and a lower capacity for clot strength measured with thromboelastometry parameters were associated with bleeding. However, a disadvantage of thromboelastography is that it gives little information on thrombin generation, which will determine the properties of a clot [40].

\section{Conclusion}

This study shows that thrombin generation can be determined during CPB despite the high heparinization level, it reflects the hemostatic capacity of the patient better than the clotting-based assays and might better predict bleeding after surgery when performed intraoperatively in platelet-rich plasma than other tests. CAT might be considered as a screening test to assess the hemostatic condition intraoperatively. More prospective studies are required to investigate whether intraoperative thrombin generation measurements can lead to proactive interventions like administration of substitution agents or blood components, which could reduce blood loss postoperatively. 


\section{References}

1. Chandler WL, Velan T. Estimating the rate of thrombin and fibrin generation in vivo during cardiopulmonary bypass. Blood 2003;101:4355-4362.

2. Hunt BJ, Parratt RN, Segal HC, Sheikh S, Kallis P, Yacoub M. Activation of coagulation and fibrinolysis during cardiothoracic operations. Ann Thorac Surg 1998;65:712-718.

3. Woodman RC, Harker LA. Bleeding complications associated with cardiopulmonary bypass. Blood 1990;76:1680-1697.

4. Hardy JF, De Moerloose P, Samama M; Groupe d'intérêt en Hémostase Périopératoire. Massive transfusion and coagulopathy: pathophysiology and implications for clinical management. Can J Anaesth 2004;51:293-310.

5. De Somer F, Van Belleghem Y, Caes F, Francois K, Van Overbeke H, Arnout J, et al. Tissue factor as the main activator of the coagulation system during cardiopulmonary bypass. J Thorac Cardiovasc Surg 2002;123:951-958.

6. Despotis G, Avidan M, Eby C. Prediction and management of bleeding in cardiac surgery. J Thromb Haemost 2009;7 Suppl 1: 111-117.

7. Hemker HC, Giesen PL, Ramjee M, Wagenvoord R, Beguin S. The thrombogram: monitoring thrombin generation in platelet-rich plasma. Thromb Haemost 2000;83:589-591.

8. Despotis GJ, Summerfield AL, Joist JH, Goodnough LT, Santoro SA, Spitznagel E, et al. Comparison of activated coagulation time and whole blood heparin measurements with laboratory plasma anti-Xa heparin concentration in patients having cardiac operations. J Thorac Cardiovasc Surg 1994;108:1076-1082.

9. Coakley M, Hall JE, Evans C, Duff E, Billing V, Yang L, et al. Assessment of thrombin generation measured before and after cardiopulmonary bypass surgery and its association with postoperative bleeding. J Thromb Haemost 2011;9:282-292.

10. Al Dieri R, De Laat B, Hemker HC. Thrombin generation: what have we learned? Blood Rev 2012;26:197-203.

11. Solomon C, Rahe-Meyer N, Sorensen B. Fibrin formation is more impaired than thrombin generation and platelets immediately following cardiac surgery. Thromb Res 2011;128:277-282.

12. Hemker HC, Beguin S. Phenotyping the clotting system. Thromb Haemost 2000;84:747-751.

13. Adams M. Assessment of thrombin generation: useful or hype? Semin Thromb Hemost 2009;35:104-110.

14. Baglin T. Unraveling the thrombophilia paradox: from hypercoagulability to the prothrombotic state. J Thromb Haemost 2010;8:228-233.

15. van Veen JJ, Gatt A, Makris M. Thrombin generation testing in routine clinical practice: are we there yet? Br J Haematol 2008;142:889-903.

16. Bosch YPJ, Al Dieri R, Ten Cate H, Nelemans PJ, Bloemen S, Hemker HC, et al. Preoperative thrombin generation is predictive for the risk of blood loss after cardiac surgery: a research article. J Cardiothorac Surg 2013;8:154.

17. Hemker HC, Giesen P, Al Dieri R, Regnault V, De Smedt E, Wagenvoord, R, et al. Calibrated automated thrombin generation measurement in clotting plasma. Pathophysiol Haemost Thromb 2003;33:4-15.

18. Bevan DH. Cardiac bypass haemostasis: putting blood through the mill. Br J Haematol 1999;104:208-219.

19. Bloemen S, Hemker HC, Al Dieri R. Large inter-individual variation of the pharmacodynamic effect of anticoagulant drugs on thrombin generation. Haematologica 2013;98:549-554.

20. Robert S, Ghiotto J, Pirotte B, David JL, Masereel B, Pochet L, et al. Is thrombin generation the new rapid, reliable and relevant pharmacological tool for the development of anticoagulant drugs? Pharmacol Res 2009;59:160-166.

21. Al Dieri R, Alban S, Beguin S, Hemker HC. Thrombin generation for the control of heparin treatment, comparison with the activated partial thromboplastin time. J Thromb Haemost 2004;2:1395-1401. 
22. Sandset PM, Abildgaard U, Larsen ML. Heparin induces release of extrinsic coagulation pathway inhibitor (EPI). Thromb Res 1988;50:803-813.

23. Koestenberger M, Cvirn G, Nagel B, Rosenkranz A, Leschnik B, Gamillscheg A, et al. Thrombin generation determined by calibrated automated thrombography (CAT) in pediatric patients with congenital heart disease. Thromb Res 2008;122:13-19.

24. De Smed E, Wagenvoord R, Hemker HC. The technique of measuring thrombin generation with fluorogenic substrates: 3. The effects of sample dilution. Thromb Haemost 2009;101:165-170.

25. Adams MJ, Cardigan RA, Marchant WA, Grocott MP, Mythen MG, Mutch M, et al. Tissue factor pathway inhibitor antigen and activity in 96 patients receiving heparin for cardiopulmonary bypass. J Cardiothorac Vasc Anesth 2002;16:59-63.

26. Donahu BS, Gailani D, Mast AE. Disposition of tissue factor pathway inhibitor during cardiopulmonary bypass. J Thromb Haemost 2006;4:1011-1016.

27. Fischer $\mathrm{T}$, Kuppe $\mathrm{H}$, Koster $\mathrm{A}$. Impact of heparin management on release of tissue factor pathway inhibitor during cardiopulmonary bypass. Anesthesiology 2004;100:1040.

28. Sun LB, Utoh J, Kunitomo C, Tsurusaki S, Tagami S, Hirata H, et al. Altered plasma antigen levels of tissue factor pathway inhibitor during open-heart surgery. Surg Today 2000;30:122-126.

29. Karkouti K, McCluskey SA, Syed S, Pazaratz C, Poonawala H, Crowther MA. The influence of perioperative coagulation status on postoperative blood loss in complex cardiac surgery: a prospective observational study. Anesth Analg 2010;110:1533-1540.

30. Koster A, Despotis G, Gruendel M, Fischer T, Praus M, Kuppe H, et al. The plasma supplemented modified activated clotting time for monitoring of heparinization during cardiopulmonary bypass: a pilot investigation. Anesth Analg 2002;95:26-30.

31. Kitchen S, lampietro R, Woolley AM, Preston FE. Anti Xa monitoring during treatment with low molecular weight heparin or danaparoid: inter-assay variability. Thromb Haemost 1999;82:1289-1293.

32. Kitchen S, Theaker J, Preston FE. Monitoring unfractionated heparin therapy: relationship between eight anti-Xa assays and a protamine titration assay. Blood Coagul Fibrinolysis 2000;11:137-144.

33. Kovacs MJ, Keeney M, Mackinnon K, Boyle E. Three different chromogenic methods do not give equivalent anti-Xa levels for patients on therapeutic low molecular weight heparin (dalteparin) or unfractionated heparin. Clin Lab Haematol 1999;21:55-60.

34. Greaves M; Control of Anticoagulation Subcommittee of the Scientific and Standardization Committee of the International Society of Thrombosis and Haemostasis. Limitations of the laboratory monitoring of heparin therapy. Scientific and Standardization Committee Communications: on behalf of the Control of Anticoagulation Subcommittee of the Scientific and Standardization Committee of the International Society of Thrombosis and Haemostasis. Thromb Haemost 2002;87:163-164.

35. Despotis GJ, Gravlee G, Filos K, Levy J. Anticoagulation monitoring during cardiac surgery: a review of current and emerging techniques. Anesthesiology 1999;91:1122-1151.

36. Culliford AT, Gitel SN, Starr N, Thomas ST, Baumann FG, Wessler S, et al. Lack of correlation between activated clotting time and plasma heparin during cardiopulmonary bypass. Ann Surg 1981;193:105-111.

37. Eikelboom JW,Hirsh J. Monitoring unfractionated heparin with the aPTT: time for a fresh look. Thromb Haemost 2006;96:547-552.

38. Hirsh J, Warkentin TE, Shaughnessy SG, Anand SS, Halperin JL, Raschke R, et al. Heparin and lowmolecular-weight heparin: mechanisms of action, pharmacokinetics, dosing, monitoring, efficacy, and safety. Chest 2001;119(1 Suppl):64S-94S.

39. Davidson SJ, Burman JF, Philips SM, Onis SJ, Kelleher AA, De Souza AC, et al. Correlation between thrombin potential and bleeding after cardiac surgery in adults. Blood Coagul Fibrinolysis 2003;14:175179.

40. Hemker HC, Al Dieri R, Beguin S. Thrombin generation assays: accruing clinical relevance. Curr Opin Hematol 2004;11:170-175. 



\section{CHAPTER 6}

\section{PREOPERATIVE THROMBIN GENERATION IS PREDICTIVE FOR THE RISK OF BLOOD LOSS AFTER CARDIAC SURGERY}

Yvonne Bosch

Raed Al Dieri

Hugo ten Cate

Patty Nelemans

Saartje Bloemen

Coenraad Hemker

Patrick Weerwind

Jos Maessen

Baheramsjah Mochtar

\section{Based on:}

Preoperative thrombin generation is predictive for the risk of blood loss after cardiac surgery: a research article. Journal of Cardiothoracic Surgery 2013, 8:154. 


\section{Abstract}

Objectives: In this study the value of thrombin generation parameters measured by the Calibrated Automated Thrombography for prediction of blood loss after cardiac surgery with cardiopulmonary bypass was investigated.

Methods: Thirty male patients undergoing first-time coronary artery bypass grafting were enrolled. Blood samples were taken pre-bypass before heparinization (T1) and 5 min after protamine administration (T2). Thrombin generation was measured both in platelet-rich plasma and in platelet-poor plasma. Besides thrombin generation measurements, activated clotting time, hematocrit, hemoglobin, platelet number, fibrinogen, antithrombin, D-dimers, prothrombin time and activated partial thromboplastin time were determined. Blood loss was measured and the amount of transfusion products was recorded postoperatively until 20 hours after surgery. Patients were divided into two groups based on the median volume of postoperative blood loss (group 1: patients with median blood loss $<930 \mathrm{~mL}$; group 2: patients with median blood loss $\geq 930 \mathrm{~mL}$ ).

Results: On T1, patients of group 2 had a significantly lower endogenous thrombin potential and peak thrombin ( $p<0.001$ and $p=0.004$ respectively) in platelet-rich plas$m a$, a significantly lower endogenous thrombin potential $(p=0.004)$ and peak thrombin $(p=0.014)$ in platelet-poor plasma, and a lower platelet count $(p=0.002)$. On T2 both endogenous thrombin potential and peak thrombin remain significantly lower ( $p=0.011$ and $p=0.010$ ) in group 2 , measured in platelet-rich plasma but not in plateletpoor plasma. In addition, platelet number remains lower in group 2 after protamine administration $(p=0.002)$.

Conclusions: The key finding is that the Calibrated Automated Thrombography assay, performed preoperatively, provides information predictive for blood loss after cardiac surgery.

Keywords: bleeding, blood (anti)coagulation, blood loss, thrombin generation, CABG, CPB 


\section{Introduction}

Blood loss and thrombotic incidents still belong to the most frequent and feared complications in cardiac surgery with cardiopulmonary bypass (CPB) [1], despite of preoperative evaluation of the hemostatic condition by assessment of medical history and common coagulation tests $[2,3]$. Disturbed hemostasis can be attributed to abnormal thrombin generation (TG), platelet dysfunction and excessive fibrinolysis. Anticoagulation with high doses of heparin may result in further hemostasis impairment. Currently, tools to adequately predict hemostasis and therefore to control blood loss are lacking. Although it was already shown that the use of thromboelastography (TEG) may help to reduce blood product consumption, studies investigating the relationship between coagulation tests, like TEG, hemostatic variables and bleeding are inconsistent in demonstrating a clear predictive value for the volume of blood loss after CPB $[2,4$, 5]. In part, this inconsistency may be due to the fact that TEG only shows clot formation, not the total amount of thrombin, which plays a central role in hemostasis [6]. In this study, we aimed at investigating the value of thrombin generation parameters as measured by the Calibrated Automated Thrombography (CAT) [6] for the prediction of blood loss after cardiac surgery. This assay displays not only the moment blood starts to clot but also the amount of thrombin that forms in clotting plasma with or without platelets. Conceptually, results of CAT measurements correlate with impaired hemostasis if too low, and with thrombosis risk if too high $[7,8]$. CAT might improve the timely identification of hemostatic problems underlying a bleeding tendency perioperatively. In this study, we hypothesize that CAT measurement, in comparison with conventional methods, may predict better the risks of blood loss and the need for transfusion products. Improved prediction could contribute to safer anticoagulation, since CAT quantifies the (anti) coagulant effects during CPB, to more optimal hemostasis, to a decrease in excessive bleeding and to improved transfusion management.

\section{Materials and Methods}

\section{Study population}

The study was approved by the local ethics committee (METC, aZM/UM), and written informed consent was obtained. In total, 30 male patients undergoing elective firsttime coronary artery bypass grafting (CABG) were enrolled. Exclusion criteria were age $<18$ years, use of preoperative anticoagulation (excepting aspirin) within the preceding 5 days, known coagulopathy, impaired renal function, liver diseases resulting in elevated liver function tests and redo surgery. 


\section{Anticoagulation and $C P B$}

An initial dose of $300 \mathrm{IU} / \mathrm{kg}$ of body weight of heparin (Heparin Leo, Leo Pharmaceutical Products BV, Weesp, the Netherlands) was injected into a central venous line before initiation of CPB. The kaolin activated clotting time (ACT) was measured and, if the value was $\geq 400 \mathrm{~s}, \mathrm{CPB}$ was initiated. If necessary, additional heparin was added. At the end of CPB, heparin was reversed by protamine chloride (Valeant Pharmaceuticals, Eschborn, Germany) at a 1:1 ratio of the loading dose. All components of the CPB system were poly-2-methoxyethylacrylate coated (Terumo). The priming of the CPB circuit included 1,300 mL of $4 \%$ Gelofusine, $200 \mathrm{~mL} 20 \%$ mannitol, $100 \mathrm{~mL} 20 \%$ human albumin, $50 \mathrm{~mL} 8.4 \% \mathrm{NaHCO}_{3}$. and 6,500 IU heparin Leo. Retrograde autologous priming was used in most of the cases to reduce the priming volume by $200-500 \mathrm{ml}$, resulting in less hemodilution. Normothermic perfusion was used during CPB. Pericardial, pleural and residual blood of the CPB circuit after termination of CPB was drained and washed with a cell saver device. The transfusion trigger during CPB was set at a hematocrit below $23 \%$.

\section{Blood samples}

Blood samples were taken at: T1) pre-bypass before heparin administration; and, T2) 5 min after protamine administration. T1 measurements will give information about the predictive value of the parameters prior to hemostatic interventions related to CPB which could suggest differences in baseline hemostatic capacity of the blood between the patients, whereas T2 measurements may give a prediction of blood loss after CPB including the influence of all hemostatic disturbances related to CPB. Blood samples were withdrawn from the arterial line after discarding the first $10 \mathrm{~mL}$.

\section{Thrombin generation}

Arterial blood samples were collected into trisodium citrate and analyzed with CAT as previously reported [9]. TG was measured both in platelet-rich plasma (PRP) and in platelet-poor plasma (PPP) to see the influence of platelets on TG. PRP was used within $1 \mathrm{~h}$ after blood withdrawal. PPP was stored at $-80^{\circ} \mathrm{C}$ until further analysis. The CAT assay was measured in a prewarmed plate fluorometer (Ascent reader, Thermolabsystems OY, Helsinki, Finland). To each well, $80 \mu$ of plasma was added in combination with the trigger: for PPP, the trigger was $30 \mathrm{pM}$ of recombinant tissue factor (rTF) and phospholipid vesicles in Hepes-buffered saline, for PRP the trigger was $20 \mu$ of $3 \mathrm{pM}$ of rTF without added phospholipids. Data were analyzed using Thrombinoscope ${ }^{\mathrm{TM}}$ software (Thrombinoscope bv, Maastricht, the Netherlands). CAT parameters which are 
used to determine correlation of TG with postoperative blood loss are: 1) lag time (min): the initiation phase of clotting which equals the clotting time; 2) peak height $(\mathrm{nM})$ : the maximal amount of thrombin formed; 3) endogenous thrombin potential (ETP) $\left(\mathrm{nM}^{*} \mathrm{~min}\right)$ : the area under the curve representing thrombin generation and decay in time; and 4) time to peak ( $\mathrm{min})$ : the time needed to achieve the peak height.

\section{ACT and laboratory tests}

Besides CAT measurements, ACT and laboratory parameters hematocrit, hemoglobin, thrombocytes, fibrinogen, antithrombin, D-dimers, prothrombin time (PT) and activated partial thromboplastin time (aPTT) were determined at both time points to assess patient's hemostatic profile.

\section{Postoperative blood loss}

Blood loss was measured at fixed time points postoperatively determined by chest tube drainage after closing the chest until 20 hours after surgery. The amount of transfusion products (packed red cells, thrombocytes or fresh frozen plasma) was recorded until 20 hours after surgery.

\section{Statistical analysis}

Data were defined as continuous or categorical variables. Categorical variables are expressed as percentages and continuous variables as mean \pm standard deviation (SD). Patients were divided into two groups with high versus low blood loss, where the median value of blood loss volume at 20 hours postoperatively was used as cut-off value. Both groups were compared with respect to patient characteristics, medication used pre- and intraoperatively, infusion solutions, mean time on bypass, amount of transfusion products, mean values of CAT parameters and other laboratory parameters. The Chi-square test was used for comparison of proportions and the Student's t-test for independent samples for comparison of mean values. The ability of CAT and laboratory parameters to discriminate between the two groups was also evaluated by the construction of a receiver operating characteristic (ROC) curve. The corresponding area under the curve (AUC) with $95 \%$ confidence interval was used to quantify the predictive value of the parameters. Multivariate linear regression analysis was performed to evaluate the independent effects of parameters. Statistical analysis was performed with SPSS for Windows 16.0 (SPSS, Inc., Chicago, IL, USA). P-values $\leq 0.05$ were considered to indicate statistical significance. 


\section{Results}

Thirty male patients were enrolled into the study. One patient was excluded because an exceptionally long term adhesiolysis was necessary to achieve access to the heart resulting in excess blood loss.

Table 1: Distribution of patient characteristics and perioperative variables according to postoperative blood loss.

\begin{tabular}{|c|c|c|c|c|}
\hline & $\begin{array}{l}\text { All patients } \\
\mathrm{N}=29\end{array}$ & $\begin{array}{l}\text { Group } 1 \\
\mathrm{~N}=14\end{array}$ & $\begin{array}{l}\text { Group } 2 \\
\mathrm{~N}=15\end{array}$ & P-value \\
\hline Age (years) & $65 \pm 10$ & $67 \pm 9$ & $64 \pm 11$ & 0.423 \\
\hline Aspirin exp. preop. $n(\%)$ & $27(93.1 \%)$ & $14(100 \%)$ & $13(86.7 \%)$ & 0.157 \\
\hline \multicolumn{5}{|l|}{ Intraoperatively } \\
\hline Tranexam. acid $n(\%)$ & $14(48.3 \%)$ & $9(64.3 \%)$ & $5(33.3 \%)$ & 0.096 \\
\hline Heparin-total (IU/kg) & $336 \pm 54$ & $361 \pm 66$ & $314 \pm 26$ & 0.023 \\
\hline Protamine-total (mg/kg) & $3.35 \pm 0.52$ & $3.55 \pm 0.61$ & $3.17 \pm 0.37$ & 0.048 \\
\hline Crystalloids (mL/kg) & $16.9 \pm 6.5$ & $19.8 \pm 6.4$ & $14.2 \pm 5.6$ & 0.018 \\
\hline Colloid $(\mathrm{mL} / \mathrm{kg})^{*}$ & $3.9(0-16.3)$ & $0.0(0.0-13.2)$ & $5.7(0-16.3)$ & 0.084 \\
\hline PRC (units)* & $0.0(0.0-1.0)$ & $0.0(0.0-1.0)$ & $0.0(0.0-1.0)$ & 0.591 \\
\hline FFP (units) & $0 \pm 0$ & $0 \pm 0$ & $0 \pm 0$ & 1 \\
\hline PLT (units)* & $0.0(0.0-1.0)$ & $0.0(0.0-1.0)$ & $0.0(0.0-0.0)$ & 0.301 \\
\hline PRC cell saver $(\mathrm{mL} / \mathrm{kg})$ & $5.7 \pm 2.0$ & $5.0 \pm 1.3$ & $6.4 \pm 2.3$ & 0.048 \\
\hline Time on bypass (min) & $76 \pm 20$ & $70 \pm 18$ & $81 \pm 22$ & 0.160 \\
\hline Time X-clamp (min) & $48 \pm 16$ & $45 \pm 14$ & $52 \pm 18$ & 0.259 \\
\hline \multicolumn{5}{|l|}{ Postoperatively } \\
\hline PRC (units)* & $1.00(0.0-5.0)$ & $0.0(0.0-2.0)$ & $2.0(0.0-2.0)$ & 0.002 \\
\hline FFP (units)* & $0.0(0.0-2.0)$ & $0.0(0.0-0.0)$ & $0.0(0.0-2.0)$ & 0.164 \\
\hline PLT (units)* & $0.0(0.0-1.0)$ & $0.0(0.0-0.0)$ & $0.0(0.0-1.0)$ & 0.020 \\
\hline Rethoracotomy $n(\%)$ & $1(3.4 \%)$ & $0(0 \%)$ & $1(6.7 \%)$ & 0.326 \\
\hline
\end{tabular}

Data shown are mean \pm SD. Comparison between the groups was done with student $\mathrm{t}$-test; ${ }^{*}$ median and range are presented instead of mean and SD; FFP = fresh frozen plasma; PLT = platelets; $P R C=$ packed red cells.

The distribution of age, preoperative use of aspirin, and intraoperative data relating to the 29 patients are shown in Table 1. In addition to presenting data of the total group, we divided patients in two groups using as cut-off value the median volume of postoperative blood loss until 20 hours (group 1: patients with median blood loss <930 mL; group 2: patients with median blood loss $\geq 930 \mathrm{~mL}$ ). the actual blood loss in group 1 was $620 \mathrm{~mL}$, and $1205 \mathrm{~mL}$ in group 2. The sample size was based on feasibility considerations. Based on this sample size and the observed standard deviation in group 1 $(\mathrm{sd}=-/+160 \mathrm{~mL})$ the power to detect a clinically relevant increase in mean blood loss by at least $50 \%$ (from $620 \mathrm{~mL}$ to $930 \mathrm{~mL}$ ) was higher than $90 \%$.

Differences in both medication and infusion (Table 1) could result in changes in the hemostatic capacity of the blood leading to differences in thrombin generation 
and, as a consequence, in blood loss. There was no significant difference between aspirin exposure preoperatively between the two groups. Patients in group 2 were infused less crystalloids, and more autologous packed red cells derived from intraoperative cell salvage. Moreover, this group was administered less heparin (total amount of both pre and during (PB) and as a consequence less protamine. Time of bypass and aorta cross clamping were increased in group 2, but differences were not statistically significant.

Thrombin generation parameters measured in platelet-rich and platelet-poor plasma

The mean values ( \pm SD) of TG parameters on T1 and T2 in both PRP and PPP in group 1 versus group 2 are presented in Table 2 . Patients of group 2 had a significantly lower ETP and peak thrombin both on T1 and T2. Between groups differences in mean values for lag time and time to peak were smaller and not statistically significant. The distributions of ETP and peak values measured in PRP and PPP within groups are shown in Figures 1 and 2.

The AUCs as measures for ability to discriminate between the groups were highest for ETP and peak in PRP at T1 with AUC (95\% Cl) of $0.881(0.741-1.021)$ and 0.855 (0.697-1,013) respectively.

Laboratory tests

The mean values of hemoglobin, hematocrit, platelet number, fibrinogen, D-dimers, antithrombin, PT, aPTT, and ACT at T1 and T2 are shown in Table 3. A significant difference between the groups was only found for platelet number $(p=0.002)$ with lower platelet count in group 2. Differences were also seen for fibrinogen and D-dimers with lower mean values in the group with blood loss $\geq 930 \mathrm{~mL}$, but only the difference in fibrinogen at T2 achieved statistical significance $(p=0.045)$. Furthermore, it can also be observed that patients with high blood loss had significantly lower hematocrit (\%) at T2. 


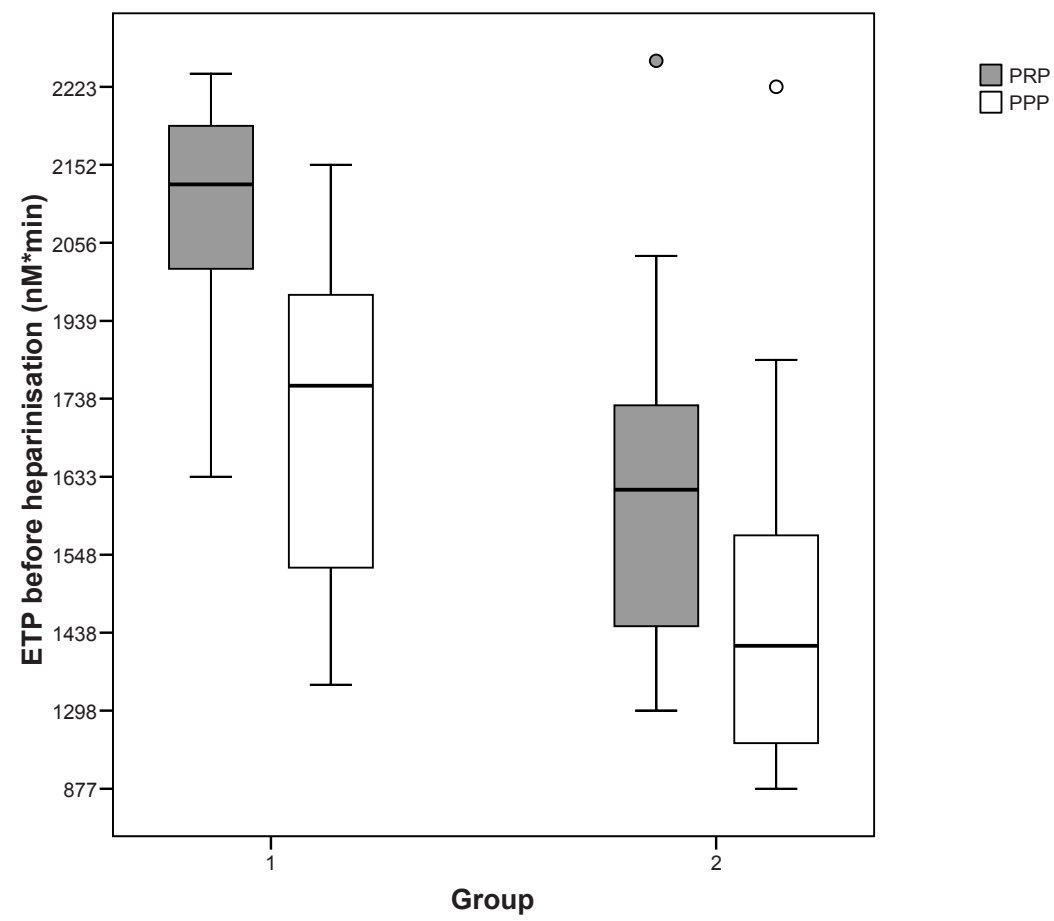

Figure 1: ETP on T1 in PRP and PPP in both groups.

Boxplot showing median, interquartile range and range of ETP measured before heparinization in patients of group 1 and 2 on $x$-axis. P-value of comparison of ETP in PRP between the groups is $<0.001, p$-value of comparison of ETP in PPP between the groups is 0.004 ; ETP=endogenous thrombin potential; PPP=platelet poor plasma; PRP=platelet rich plasma.

The highest AUC values were observed for platelet number with an AUC $(95 \% \mathrm{Cl})$ of $0.834(0.690-0.979)$ at T1 and an AUC $(95 \% \mathrm{Cl})$ of $0.857(0.705-1.009)$ at T2. It can also be observed that the AUC of hemoglobin and hematocrit is higher at T2 than at T1 with AUC $(95 \% \mathrm{Cl})$ of $0.714(0.517-0.912)$; and $0.732(0.540-0.924)$, respectively. AUCs for these parameters at T1 are low. The AUCs associated with fibrinogen and Ddimers are not impressive at both time points with AUCs ranging from 0.547 to 0.703 , and the AUC of the variables, aPTT, PT and ACT are below 0.600 .

Independent effects of platelet number and CAT parameters

A multivariate linear regression model with both platelet number and ETP/peak as independent variables and blood loss as dependent variable was performed to evaluate the independent effects of TG parameters after adjustment for differences in platelet number between group 1 and 2 . These analyses indicate that higher values of 
CAT parameters remain associated with lower blood loss. Results for measurements in PRP on T1 are shown in Tables 4 and 5, and results for the other measurements are similar.

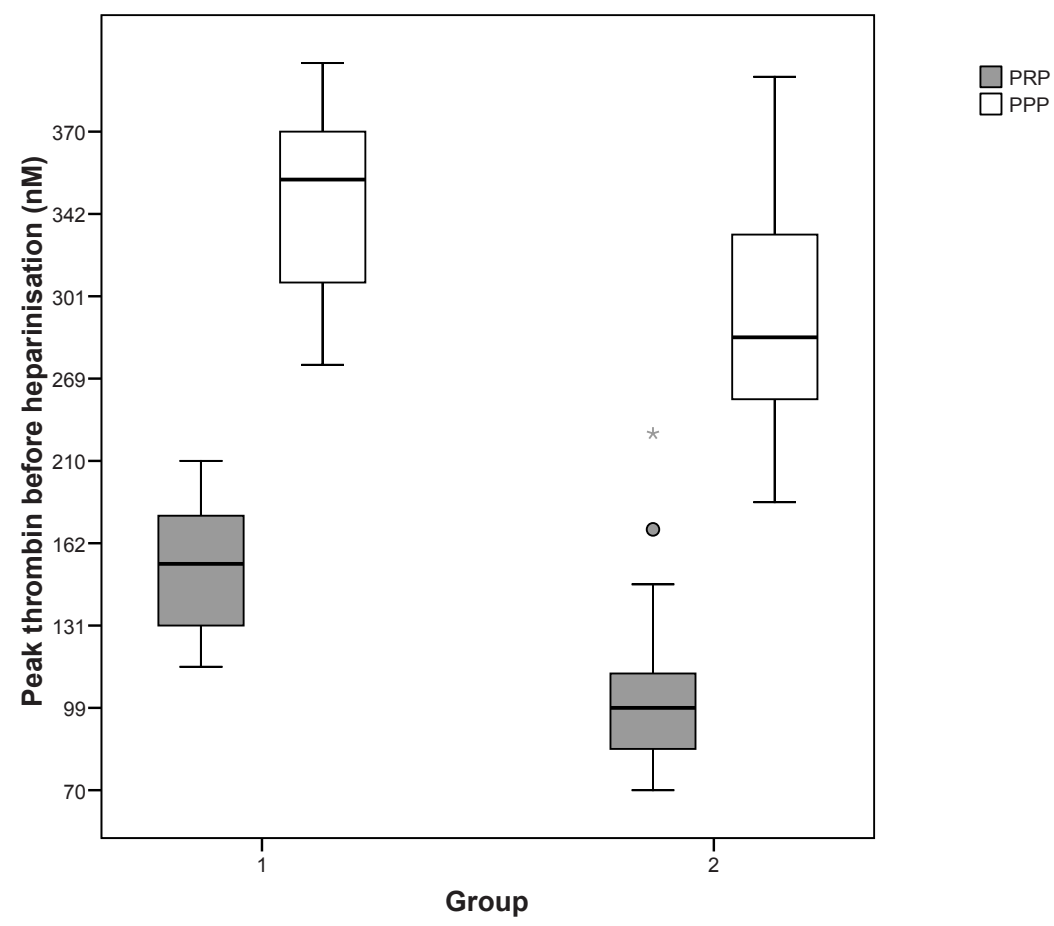

Figure 2: Peak thrombin on T1 in PRP and PPP in both groups.

Boxplot showing median, interquartile range and range of peak thrombin measured before heparinization in patients of group 1 and 2 on x-axis. P-value of comparison of peak thrombin in PRP between the groups is $0.004, p$-value of comparison of peak thrombin in PPP between the groups is 0.014; PPP=platelet poor plasma; PRP=platelet rich plasma. 


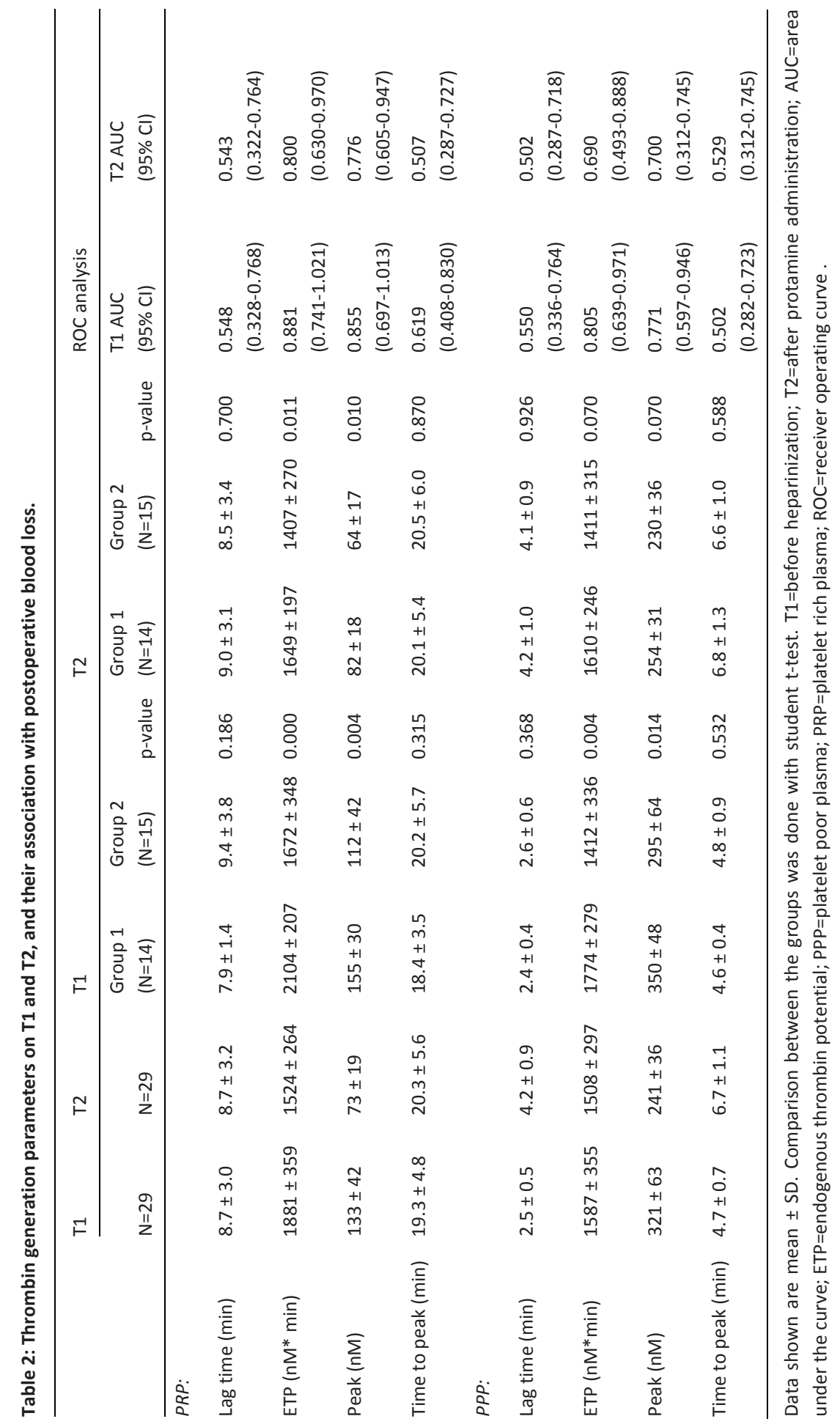




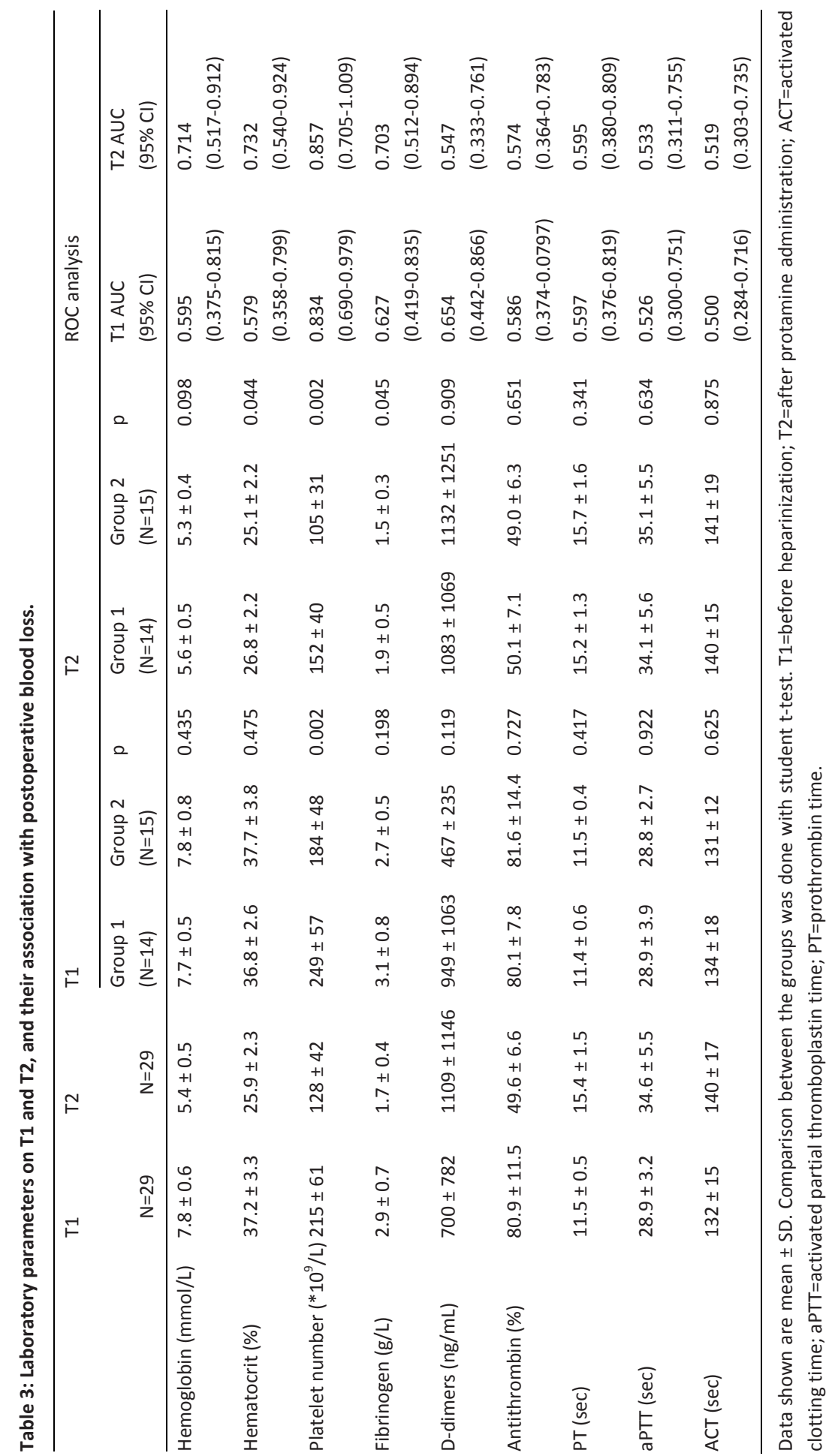


Table 4: Independent effects of platelets and ETP in PRP on blood loss at T1 by linear regression analysis.

\begin{tabular}{lllllll}
\hline \multicolumn{5}{l}{ Univariate } & \multicolumn{5}{l}{ Multivariate } \\
\hline & B unstandardized & B standardized & p-value & B unstandardized & B standardized & p-value \\
\hline ETP $(\mathrm{nM})$ & -0.429 & -0.401 & 0.031 & -0.322 & -0.301 & 0.144 \\
$\begin{array}{l}\text { Platelet number } \\
\left(* 10^{9} / \mathrm{L}\right)\end{array}$ & -2.223 & -0.354 & 0.060 & -1.325 & 0.211 & 0.301 \\
\hline
\end{tabular}

$B=$ regression coefficient; ETP=endogenous thrombin potential

Table 5: Independent effects of platelets and peak-thrombin in PRP on blood loss at T1 by linear regression analysis.

\begin{tabular}{lllllll}
\hline \multicolumn{5}{c}{ Univariate } & \multicolumn{3}{l}{ Multivariate } \\
\hline & B unstandardized & B standardized & p-value & B unstandardized & B standardized & p-value \\
\hline Peak $(\mathrm{nM})$ & -0.290 & -0.319 & 0.092 & -2.026 & -0.223 & 0.253 \\
$\begin{array}{l}\text { Platelet number } \\
\left(* 10^{9} / \mathrm{L}\right)\end{array}$ & -2.223 & -0.354 & 0.060 & -1.737 & -0.276 & 0.159 \\
\hline
\end{tabular}

$B=$ regression coefficient

\section{Discussion}

The present data suggest that the CAT variables ETP and peak thrombin correlate well with the clinically observed bleeding tendency postoperatively in cardiac surgical patients. Patients of group 2 had a significantly lower ETP and peak thrombin, both pre (PRP and PPP) and post CPB (only PRP), than patients in group 1. Concerning the other laboratory parameters, platelet number was the only individual variable measured preCPB predicting blood loss postoperatively. Post CPB, besides low fibrinogen a low platelet number was also predictive for blood loss.

Recently Coakley and co-workers demonstrated that TG both pre- and postoperatively could potentially be used to identify patients at an increased risk of bleeding post-CPB [2]. However, they only measured TG in PPP, excluding the influence of platelets, which play a very important role in maintaining normal hemostatic function. It is more representative of the in vivo situation to include the procoagulant functions of platelets [10].

Conventional clot-based methods, have the disadvantage of measuring only the moment of clot formation and ensuing changes in the properties of the clot. Clot formation is only one of the many functions of thrombin and not necessarily the most important one. Thrombin also activates platelets, and furthermore, besides procoagulant effects it has also anticoagulant effects following its binding to thrombomodulin. 
Ex vivo TG tests, like CAT, measure the hemostatic function of the blood, determined by simultaneous prothrombin activation and thrombin inactivation [9]: it measures the remaining capacity of blood to generate a thrombin burst indicating an increased risk of thrombosis or bleeding [11]. In contrast, in vivo TG, revealed by products like prothrombin fragment $\mathrm{F} 1+2$, thrombin antithrombin complex, and D-dimers, provides indications of TG that has already occurred [12] at the moment of blood collection.

Since the lag time represents the same aspect as in standard coagulation assays, this is comparable with aPTT and PT. Our results showed that both aPTT and PT as well as lag time were not different between the groups. These tests terminate with endpoints that occur with less than $5 \%$ of the reaction complete [13]. Apparently, the present data strengthen the notion that it is not the initiation phase of clotting but the propagation phase that determines hemostasis and blood loss after cardiac surgery.

Time to peak, also a time dependent variable, also did not show any significance in predicting blood loss. Peak thrombin and ETP, reflecting the amount of thrombin generated, give a more accurate evaluation of coagulability.

In group 2, patients had a lower mean platelet number both before heparinization and after protamine administration. Platelets are important in the blood coagulation process and play an important role in TG by providing a procoagulant membrane surface, and hence supporting the formation of more thrombin [14]. We observed in this study that reduced platelet number was also predictive for blood loss although the latter outcome occurs within the normal ranges of platelet number. It is worth noting however that platelet number cannot detect abnormality in the coagulation system. CAT, in contrast, is a global functional test that reflects the coagulation profile of the patient in the absence and presence of platelets. Bleeding tendency is related to the hemostatic conditions in pro- and anticoagulant factors, platelets, vessel wall and the fibrinolytic system. TG in PRP reflects the major part of this physiological clotting system, including the interaction between platelets and the clotting system.

Concerning coagulation factors we only measured fibrinogen. This factor demonstrated to be higher both pre- and postoperatively in group 1, but the difference was only statistically significant postoperatively. In the study of Coakley [2] coagulation factor levels were determined postoperatively in groups that either bled more than $1 \mathrm{~L}$ or less than 1L. Fibrinogen level was the same in both groups, in contrast with our results. Karlsson et al. [15] investigated the relationship between preoperative fibrinogen plasma concentration and postoperative bleeding after CABG surgery. Their main finding was that higher preoperative fibrinogen was correlated with less blood loss postoperatively. Despite of the important contribution of fibrinogen in the coagulation process, our study demonstrates less strong results regarding fibrinogen level compared to TG parameters. This is remarkable because, although fibrinogen is depleted before $5 \%$ of all thrombin is formed [11], thrombin bound to fibrin has a positive feed- 
back activation on the coagulation system and is protected from inactivation to the action of antithrombin and heparin [16].

Remarkable is the significantly higher dose of intraoperative heparin in group 1. Most probably a higher dose of heparin is administered in this group as a result of a relatively high platelet number in this group [17]: the concentration of heparin required to inhibit or delay coagulation is directly related to the number of platelets [18], probably due to the capacity of platelet released platelet factor 4 neutralizing heparin $[19,20]$.

Patients who bled more, were administered proportionally less crystalloids and more colloids. Colloids are known to affect clot formation by reducing fibrinogen concentration and disturbing fibrin polymerization [21] demonstrated by thromboelastography assay. Schols et al. found that colloids reduce the formation of fibrin clots but affect TG only at clinically irrelevant high concentrations [22]. The volume of colloids administered was not significantly different between the groups.

Group 2 is characterized by a higher supplementation of transfusion products. In addition, these patients had a higher intraoperative blood loss, as shown by a higher amount of PRC volume processed by cell salvage. Despotis et al. also demonstrated that greater volumes of intraoperative salvaged red cells were associated with excessive blood loss and use of blood products [23], explained by the fact that greater volumes of salvaged red cells primarily reflects excessive blood loss intraoperatively but proceeding postoperatively. Another explanation may be that extensive intraoperative cell salvage caused a significant loss of platelets and plasma resulting in postoperative bleeding.

The strength of this study is that CAT, performed preoperatively, is able to predict blood loss after cardiac surgery. Preoperative measurements (T1) exclude the hemostatic effects caused by exposure of the blood to different non-physiological conditions. The predictive value of CAT performed postoperatively (T2) for blood loss will be influenced by a lot of interventions like heparinization, fluid administration (crystalloid and/or colloid), administration of tranexamic acid and protamine, time on bypass, and transfusion product requirements intraoperatively. All these factors interfere at different levels in the hemostatic balance of the patients what makes the prediction of blood loss by CAT parameters weaker at T2 (AUCs are lower at T2). The main limitation of the study is the low number of participants $(n=30)$. P-values in the multivariate linear regression models were higher or even no longer statistically significant, possibly due to small sample size. The results should be considered as preliminary. Changes in hemostatic factors would have been more reliable in a larger number of patients. Another limitation of the study is that it could not account for variations in routine practice in the ICU that may have influenced blood loss, such as amount and timing of 
platelet and plasma transfusion. Therefore, additional larger clinical, and preferable intervention, studies are required to establish profound clinical relevance.

\section{Conclusions}

The key finding is that the Calibrated Automated Thrombography assay, performed preoperatively, provides information predictive for non-surgical blood loss after cardiac surgery, and might be considered as a standard screening test to assess the hemostatic condition preoperatively. Improved hemostatic management of patients undergoing cardiac surgery, especially high risk patients, requires more knowledge on the changes in TG, and on proactive interventions like administration of hemostaticaugmenting pharmacologic agents or blood components. Identifying patients at increased risk of bleeding will become easier because techniques for measuring TG in whole blood as a bedside method are in development, so that the outcome of a study with the CAT can, in the future, be more easily applied in clinical settings. 


\section{References}

1. Groenenberg I, Weerwind PW, Everts PAM, Maessen JG. Dutch perfusion incident survey. Perfusion 2010;25:329-336.

2. Coakley M, Hall JE, Evans C, Duff E, Billing V, Yang L et al. Assessment of thrombin generation measured before and after cardiopulmonary bypass surgery and its association with postoperative bleeding. J Thromb Haemost 2011;9:282-292.

3. Innerhofer P, Kienast J. Principles of perioperative coagulopathy. Best Pract Res Clin Anaesthesiol 2010;24:1-14.

4. Luddington RJ. Thrombelastography/thromboelastometry. Clin Lab Haematol 2005;27:81-90.

5. Reinhofer M, Brauer M, Franke U, Barz D, Marx G, Losche W. The value of rotation thromboelastometry to monitor disturbed perioperative haemostasis and bleeding risk in patients with cardiopulmonary bypass. Blood Coagul Fibrinolysis 2008;19:212-219.

6. Hemker HC, Giesen P, Al Dieri R, Regnault V, De Smedt E, Wagenvoord R et al. The calibrated automated thrombogram (CAT): a universal routine test for hyper- and hypocoagulability. Pathophysiol Haemost Thromb 2002;32:249-253.

7. Al Dieri R, Peyvandi F, Santagostino E, Giansily M, Mannucci PM, Schved JF et al. The thrombogram in rare inherited coagulation disorders: its relation to clinical bleeding. Thromb Haemost 2002;88:576582.

8. Orbe J, Zudaire M, Serrano R, Coma-Canella I, Martinez de Sizarrondo S, Rodriguez JA et al. Increased thrombin generation after acute versus chronic coronary disease as assessed by the thrombin generation test. Thromb Haemost 2008;99:382-387.

9. Hemker HC, Giesen P, Al Dieri R, Regnault V, De Smedt E, Wagenvoord R et al. Calibrated automated thrombin generation measurement in clotting plasma. Pathophysiol Haemost Thromb 2003;33:4-15.

10. Hemker HC, Beguin S. Phenotyping the clotting system. Thromb Haemost 2000;84:747-751.

11. Hemker HC, Al Dieri R, Beguin S. Thrombin generation assays: accruing clinical relevance. Curr Opin Hematol 2004;11:170-175.

12. Wong RK, Sleep JR, Visner AJ, Raasch DJ, Lanza LA, De Valeria PA et al. Thrombography reveals thrombin generation potential continues to deteriorate following cardiopulmonary bypass surgery despite adequate hemostasis. J Extra Corpor Technol 2011;43:19-25.

13. Mann KG, Brummel K, Butenas S. What is all that thrombin for? J Thromb Haemost 2003;1:1504-1514.

14. Heemskerk JW, Bevers EM, Lindhout T. Platelet activation and blood coagulation. Thromb Haemost 2002;88:186-193.

15. Karlsson M, Ternstrom L, Hyllner M, Baghaei F, Nilsson S, Jeppsson A. Plasma fibrinogen level, bleeding, and transfusion after on-pump coronary artery bypass grafting surgery: a prospective observational study. Transfusion 2008;48:2152-2158.

16. Kumar R, Beguin S, Hemker HC. The influence of fibrinogen and fibrin on thrombin generation-evidence for feedback activation of the clotting system by clot bound thrombin. Thromb Haemost 1994;72:713-721.

17. Bosch YPJ, Weerwind PW, Nelemans PJ, Maessen JG, Mochtar B. An Evaluation of Factors Affecting Activated Coagulation Time. J Cardiothorac Vasc Anesth 2012;26:563-568.

18. Conley CL, Hartmann RC, Lalley JS. The relationship of heparin activity to platelet concentration. Proc Soc Exp Biol Med 1948;69:284-287.

19. Horne MK $3^{\text {rd }}$, Chao ES. Heparin binding to resting and activated platelets. Blood 1989;74:238-243.

20. Lane DA, Denton J, Flynn AM, Thunberg L, Lindahl U. Anticoagulant activities of heparin oligosaccharides and their neutralization by platelet factor 4. Biochem J 1984;218:725-732. 
21. Innerhofer P, Fries D, Margreiter J, Klingler A, Kuhbacher G, Wachter B et al. The effects of perioperatively administered colloids and crystalloids on primary platelet-mediated hemostasis and clot formation. Anesth Analg 2002;95:858-865.

22. Schols SE, Feijge MA, Lance MD, Hamulyak K, Ten Cate H, Heemskerk JW et al. Effects of plasma dilution on tissue-factor-induced thrombin generation and thromboelastography: partly compensating role of platelets. Transfusion 2008;48:2384-2394.

23. Despotis GJ, Filos KS, Zoys TN, Hogue CW Jr, Spitznagel E, Lappas DG. Factors associated with excessive postoperative blood loss and hemostatic transfusion requirements: a multivariate analysis in cardiac surgical patients. Anesth Analg 1996;82:13-21. 



\section{CHAPTER 7}

\section{THE ASSOCIATION BETWEEN ROTATIONAL THROMBOELASTOMETRY PARAMETERS AND BLOOD LOSS AFTER CARDIAC SURGERY WITH CARDIOPULMONARY BYPASS}

Yvonne Bosch

Patrick Weerwind

Hugo ten Cate

Yvonne Henskens

Patty Nelemans

Raed Al Dieri

Jos Maessen

Baheramsjah Mochtar.

Based on:

The association between rotational thromboelastometry parameters and blood loss after cardiac surgery with cardiopulmonary bypass. Accepted for publication in the Journal of Cardiovascular and Thoracic Open. 


\section{CHAPTER 7}

\section{Abstract}

The objectives of this study were to investigate the changes in routine laboratory tests and rotational thromboelastometry (ROTEM) variables after cardiopulmonary bypass $(\mathrm{CPB})$, and to investigate the association of these variables with postoperative blood loss.

Blood was obtained at baseline, at admission to the intensive care unit (ICU), and at the first postoperative day. ROTEM tests and measurements of hemoglobin, hematocrit, platelet count and plasma fibrinogen level (Clauss method) were performed. Excessive bleeding was defined as $>1000 \mathrm{~mL}$ of chest tube drainage after surgery within 24 hours.

After CPB hematocrit was decreased by $21 \%$. Platelet count and fibrinogen level decreased more (35\% and $42 \%$ respectively). The largest change was found for clot formation time (CFT) of INTEM (+73\%). Platelet count was not reflected by maximal clot firmness (MCF) of PLATEM. The decreases in FIBTEM-MCF and fibrinogen at ICU admission were comparable. Test results outside the reference range were associated with higher median blood loss when compared to values within the reference range, with most significant association for fibrinogen $(p<0.001)$ and FIBTEM-MCF $(p=0.016)$. At baseline, the ability of ROTEM parameters to predict postoperative blood loss $>1000 \mathrm{~mL}$ is higher than the predictive value of fibrinogen $(A \cup C=0.642 ; 95 \% \mathrm{Cl}: 0.487-$ $0.796)$, but only AUCs of APTEM were significantly higher ( $p \leq 0.05)$. At ICU admission the highest AUC was 0.689 (95\% Cl: 0.549-0.828), for FIBTEM-MCF.

ROTEM could be a useful point-of-care method to monitor hemostasis because, it is a faster and more informative method, and it has a slightly higher predictive ability for blood loss compared to fibrinogen.

Key words: cardiopulmonary bypass, rotational thromboelastometry, coagulation tests, blood loss 


\section{Introduction}

Cardiac surgery is associated with intra- and postoperative bleeding $[1,2]$ due to the hemostatic disturbances related to cardiopulmonary bypass (CPB). Platelet dysfunction is often considered to be the most important acquired defect in hemostasis in the early postoperative period [3]. Besides, excessive postoperative bleeding has been attributed to increased fibrinolysis [4]. In addition, the perioperative plasma fibrinogen concentration, is associated with postoperative bleeding and transfusion requirements after CPB. Early characterization of the hemostatic defects responsible for bleeding is crucial for optimizing management of the patient. However, routine laboratory parameters provide inadequate guidance for timely and goal-directed hemostatic intervention [4]. Whole blood viscoelastic tests like thromboelastography (TEG) and rotational thromboelastometry (ROTEM) are more suitable for timely decision-making $(<15-20$ $\mathrm{min}$ ) in hemostatic interventions than tests performed in plasma. These whole blood tests, providing information on the contribution of both fibrinogen and platelets to clot formation, produce clinically relevant correlations with platelet count and plasma fibrinogen levels $[5,6]$ and exhibit a high sensitivity and specificity for detecting subnormal fibrinogen levels [5]. In addition it is postulated that whole blood tests like ROTEM better reflect disturbances occurring during CPB [7] and may therefore be helpful as a guide for transfusion therapy [8-10]. However, the precise relationship between ROTEM values and postoperative bleeding remains unclear [10-14].

The aim of this study was to investigate the changes in routine laboratory tests and ROTEM variables, with subsequent association to blood loss after cardiac surgery with CPB.

\section{Methods}

\section{Patients}

After approval by the local ethical committee (reference number: NI.34179.068.10/ MEC 10-2-095) and written informed consent, 100 patients undergoing elective cardiac surgery with cardiopulmonary bypass (CPB) were included in this prospective observational study. Written informed consents are stored in a folder. The patients were scheduled for coronary artery bypass grafting, aortic valve replacement or a combination of both. Patients with preoperative coagulation disorders, who used oral anticoagulants, clopidogrel or thrombolytics within 5 days before surgery, renal insufficiency, hepatic disorders or active sepsis/endocarditis and patients with active malignancy, were excluded from study participation. 


\section{Clinical management}

General anaesthesia was induced using weight related dosing of sufentanil and etomidate, and muscle relaxation was achieved with pancuronium bromide. General anaesthesia was maintained during surgery using propofol. An initial dose of $300 \mathrm{IU} / \mathrm{kg}$ of body weight of heparin (Heparin Leo, Leo Pharmaceutical Products BV, Weesp, the Netherlands) was injected into a central venous line before the initiation of CPB. After this loading dose, the ACT was measured and, if the value was $>400 \mathrm{~s}$, CPB was initiated. If necessary, additional heparin was added to achieve an ACT of at least $400 \mathrm{~s}$. At the end of CPB, heparin was reversed by protamine chloride (Valeant Pharmaceuticals, Eschborn, Germany) at a 1:1 ratio of the loading dose, regardless of the total heparin dosage.

The CPB system consisted of a standard closed bypass circuit with a hollow-fibre membrane oxygenator (Capiox SX18R, Terumo, Tokyo, Japan), a cardiotomy reservoir (Capiox, CXCRXA, Terumo), and an arterial line filter (Pall, LeukoGuard LCG, Pall, Portsmouth, United Kingdom). All circuit components were poly-2-methoxyethylacrylate coated (Terumo).

The standard priming of the CPB circuit included 1,300 $\mathrm{mL}$ of $4 \%$ Gelofusine (B. Braun AG, Melsungen, Germany), $200 \mathrm{~mL} 20 \%$ mannitol, $100 \mathrm{~mL} 20 \%$ human albumin and $50 \mathrm{~mL} 8.4 \%$ NaHCO3. The total volume of the CPB priming amounted to $1,650 \mathrm{~mL}$ clear fluid, containing 6,500 IU/L heparin (Heparin Leo, Leo Pharmaceutical Products BV, Weesp, the Netherlands). In most of the cases a technique to reduce the large priming volume by $200-500 \mathrm{~mL}$ was used, the so-called retrograde autologous priming (RAP), which resulted in less hemodilution.

Myocardial preservation was provided by infusing St. Thomas' No.1 solution at $4^{\circ} \mathrm{C}$ via the aortic root. Target flow rates of $\geq 2.4 \mathrm{~L} / \mathrm{min} / \mathrm{m}^{2}$ were maintained throughout the $\mathrm{CPB}$ period. Normothermic $\left(\geq 36^{\circ} \mathrm{C}\right)$ pulsatile perfusion was used during the period of cross-clamping. Mean arterial blood pressure was maintained above $70 \mathrm{mmHg}$, and, if needed phenylephrine $(0.4 \mathrm{mg} / \mathrm{ml})$ was titrated via an infusion pump. Pericardial and pleural blood was drained and washed with a cell saver device. After termination of CPB and decannulation, the residual volume of the heart-lung machine was displaced into the cell saver device (C.A.T.S., Fresenius Kabi, Germany), and transfused to the patient at the end of surgery. As antifibrinolytic therapy, all patients receive 2 gram of tranexamic acid pre-CPB and 1 gram during CPB. Transfusions of red blood cells, platelets, fresh frozen plasma or fibrinogen was according to standard procedures guided on routine laboratory results or clinical observation of bleeding. 


\section{Measurements}

Blood samples were taken, after discarding the first $10 \mathrm{~mL}$, at three time points: $\mathrm{T} 1$ ) directly before the induction of general anesthesia; T2) at admission to the ICU; and T3) at the first postoperative morning. Blood was collected in $4.0 \mathrm{~mL}$ tubes with 7.2-mg spray dried $\mathrm{K}_{2}$ EDTA for analysis of hemoglobin, hematocrit and platelet count, and in $4.5 \mathrm{~mL}, 3.2 \%$ citrate tubes (BD Vacutainer, Becton Dickinson, NJ, USA) for analysis of fibrinogen and ROTEM. Complete blood count has been carried out on a Sysmex XN9000 analyzer (Sysmex Corporation, Kobe, Japan), an automated analyzer. Fibrinogen levels were determined using the Clauss coagulation method on a Sysmex CA-7000 analyzer (Sysmex Corporation, Kobe, Japan) (Dade Thrombin Reagent, Siemens Healthcare Diagnostics Products, Marburg, Germany).

Thromboelastometric measurements were performed with a 4-channel ROTEM device (ROTEM ${ }^{\circledR}$, Pentapharm, Munich, Germany). ROTEM was performed according to the manufacturer's instruction at $37^{\circ} \mathrm{C}$. The whole blood samples were recalcified with calcium chloride, and coagulation was activated with specific reagents (TEM Innovations $\mathrm{GmbH}$, Munich, Germany) using a semi-automated electronic pipette system. Analyzed ROTEM parameters were: EXTEM clotting time (CT), clot formation time (CFT), alpha angle, maximal clot firmness (MCF) (addition of tissue factor); INTEM CT, CFT, alpha angle, MCF (addition of ellagic acid); FIBTEM CT and MCF (addition of tissue factor/ cytochalastin D), APTEM CT, CFT, alpha angle and MCF (addition of tissue factor/aprotinin). As a measure for the contribution of platelets, the ROTEM variable PLATEM was calculated by subtracting FIBTEM from EXTEM (eliminating the contribution of fibrinogen). PLATEM was also used in previous studies $[4,6,15]$. Only MCF was determined for PLATEM.

Blood loss was measured postoperatively and was determined by volume of chest tube drainage after closing the chest until 24 hours after surgery. Transfusion requirements were also recorded in the whole perioperative period until 24 hours after surgery.

\section{Statistical analysis}

A sample of 100 patients undergoing complex surgery was studied to explore the association between coagulation variables and blood loss. No formal sample size analysis was performed.

Continuous variables are described as means with standard deviation (for normally distributed data, tested by Kolmogorov-Smirnov) or as median with interquartile range (for data without normal distribution). Changes in ROTEM and routine laboratory parameters were expressed as percentage from the baseline and were calculated 
as: (end value minus baseline value/ baseline value ) *100\%. Values before and after surgery were compared with a t-test or the non-parametric Wilcoxon test for paired samples where appropriate.

In order to evaluate the association of ROTEM and routine laboratory parameters with blood loss patients were categorized into subgroups: 1 . with values within the reference range [16] of the Maastricht University Medical Centre (MUMC ${ }^{+}$(G1), and 2. with values outside the reference range of the $\mathrm{MUMC}^{+}$, indicating a lower hemostatic potential (G2). Median blood loss between the two subgroups was compared with a ttest for independent samples or with the non-parametric Mann-Whitney $U$ test where appropriate. The ability of ROTEM and routine laboratory parameters before and after surgery to predict excessive blood loss in individual patients was investigated by receiver operating characteristic (ROC) analysis and was expressed as area under the curve (AUC) with 95\% confidence intervals $(\mathrm{Cl})$. Excess bleeding was defined as $>1000$ $\mathrm{mL}$ within the initial 24 hours for two reasons. This volume of blood loss has been previously used as a definition of excessive bleeding after CPB $[17,18]$, and additionally $1000 \mathrm{~mL}$ represents the $75^{\text {th }}$ percentile of the patient population in this study. AUCs of ROTEM parameters were compared with the highest AUC of the routine laboratory parameters, to evaluate whether the difference in AUC was statistically significant. Pvalues $\leq 0.05$ were considered to indicate statistical significance. All analyses were performed using SPSS version 20 (SPSS Inc., Chicago, IL, USA), excepting comparison of AUCs, which was performed with STATA version 13. All patient related data are saved in a SPSS database.

\section{Results}

A total of 100 patients, scheduled for coronary artery bypass grafting (CABG), aortic valve replacement (AVR) or a combination of CABG/AVR, participated in this study. Three patients were excluded, one because of complicated postoperative course with three times a re-exploration with exceptional blood loss, and one patient because of the necessity to use an extracorporeal life support system for cardiac support postoperatively. Due to logistic reasons it was impossible to perform ROTEM measurements at the first postoperative day (T3) for the third patient. This made us decide to exclude this case. Demographics, clinical data and information about blood loss and transfusion requirements are shown in Table 1.

At ICU (T2) both routine laboratory tests and all ROTEM variables were changed as a consequence of the CPB procedure. The relative changes from baseline for all parameters were statistically significant $(p<0.001)$ with the exception of changes for PLATEM MCF $(p=0.922)$. At the first postoperative day (T3), all variables tended to 
normalize, however, the values were still significantly different from baseline (T1) $(p<0.001)$, with the exception of fibrinogen and PLATEM (Table 2) $(p=0.156$ and $\mathrm{p}=0.137$ respectively).

Changes in routine laboratory tests

AT ICU admission hemoglobin and hematocrit decreased by $22 \%$ and $21 \%$ respectively, whereas the decreases of platelet count and fibrinogen were higher (35\% and $42 \%$ respectively). Fibrinogen was the only variable that returned to baseline at the first postoperative day (Table 2).

\section{Changes in ROTEM variables}

Table 2 shows that the most pronounced change compared with baseline was an increase in CFT in all assays (EXTEM 59\%, INTEM 73\%, and APTEM 57\%) at admission to the ICU, indicating that the most substantial decline in clot formation seems to take place after the initiation phase of clotting (indicated by CT). The increase in CFT was much higher in INTEM than in EXTEM, at both admission to the ICU and the first postoperative day. CTs of EXTEM, FIBTEM and APTEM were also increased. In addition, INTEM CT had the smallest change of all variables at admission (only 5\%), but increased further the first postoperative day ( $7 \%$ increase compared with baseline). Changes in alpha angle and MCF were more or less comparable between EXTEM and INTEM. The reduction in MCF of EXTEM and INTEM was not that robust as the reduction in FIBTEM MCF (47\%), the latter indicating a considerable reduction in the contribution of fibrinogen to clot strength. The results for APTEM were comparable with EXTEM, with only a slightly smaller change. There were no differences in PLATEM MCF between baseline and the postoperative measurements. 
Table 1: Demographic and clinical variables, transfusion requirements and blood loss of all patients.

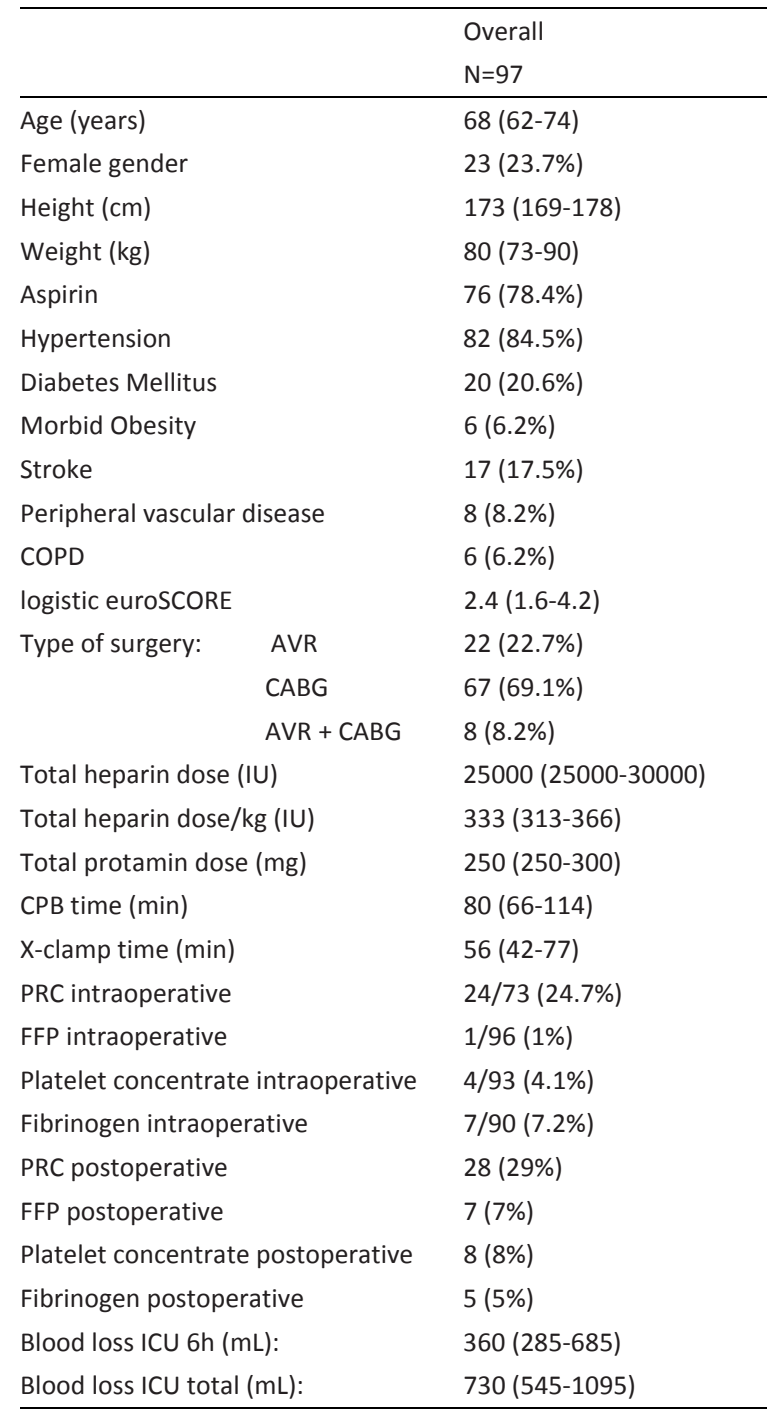

Data are represented as median and interquartile range; $A V R=$ aortic valve replacement; $C A B G=$ coronary artery bypass grafting; $C P B=$ cardiopulmonary bypass; $P R C=$ packed red cells; $\mathrm{FFP}=$ fresh frozen plasma 


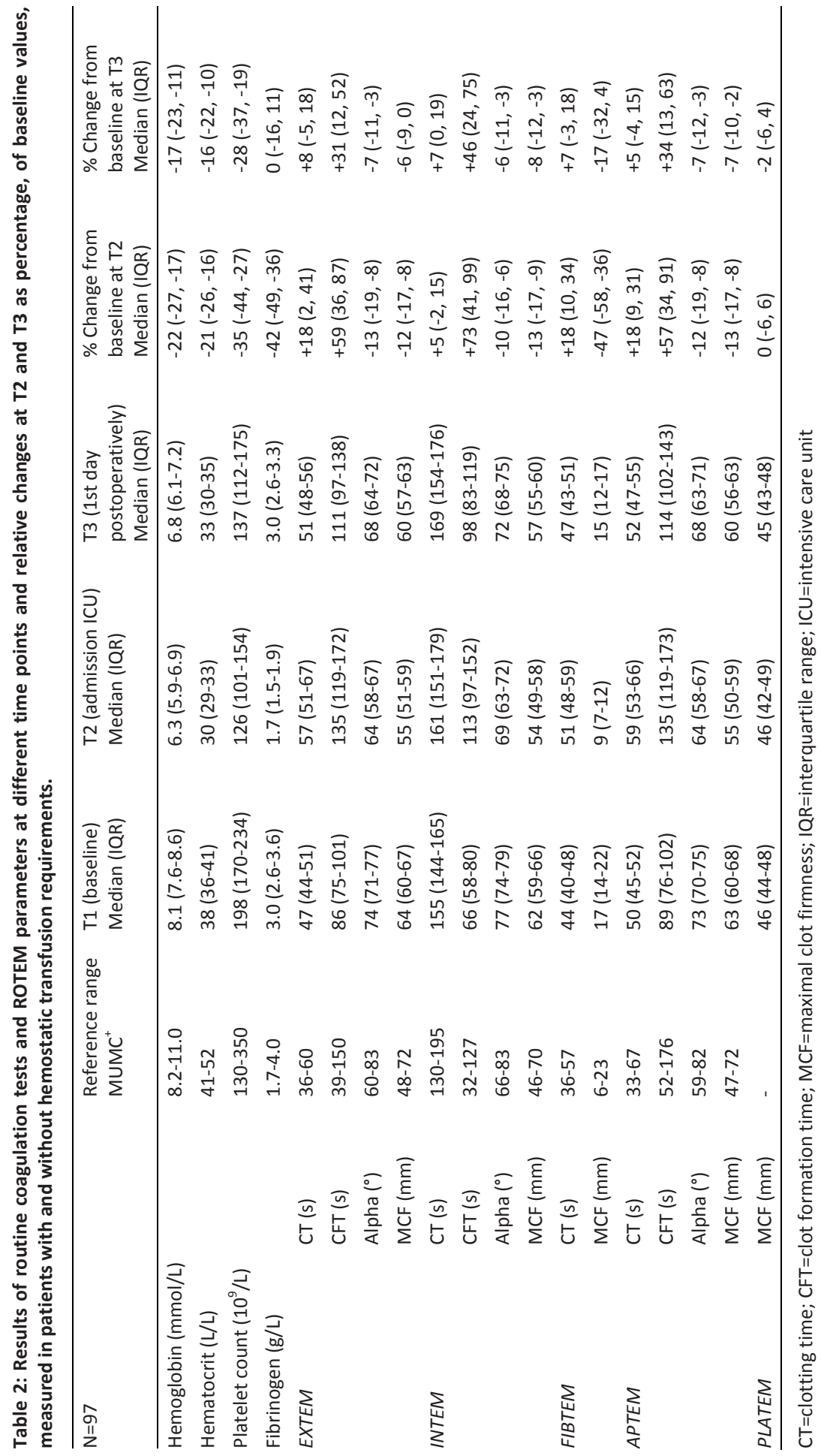


Association of hemostatic variables with postoperative blood loss

For variables measured upon admission to the ICU, patients were categorized into subgroups according to whether the value was within or outside the reference range. With respect to ROTEM parameters, values remained within the reference range for the majority of patients. Median blood loss was higher in the subgroups with values outside the reference range (G2) when compared with the subgroups with values within the reference range (G1), but statistically significant differences were only found for the parameters fibrinogen, EXTEM alpha, INTEM CT, FIBTEM CT/MCF, and APTEM CT (Table 3).

Receiver operating characteristic (ROC) analysis was performed to assess the capacity of ROTEM and routine laboratory tests in predicting blood loss of more than $1000 \mathrm{~mL}$. We restricted the analysis to patients without treatment with hemostatic transfusion requirements during the whole perioperative period, since any hemostatic transfusion could possibly affect chest tube drainage and disturb the independent influence of the routine laboratory/ROTEM parameter on blood loss. A total of 78 patients were included in this analysis (Table 4). AUCs of routine laboratory parameters were highest for plasma fibrinogen both at baseline and ICU admission ( $A \cup C=0.642$ and $A \cup C=0.646$, respectively). Concerning the ROTEM assays, CFT, alpha and MCF values at baseline were predictive for blood loss $>1000 \mathrm{~mL}$. The alpha angles showed highest AUCs (AUC $\geq 0.746$ ). At ICU admission, CT INTEM and MCF FIBTEM were significantly associated with increased bleeding ( $A \cup C=0.669$ and $A U C=0.689$, respectively). Furthermore, AUCs of all ROTEM parameters were compared with the AUC of fibrinogen at baseline and at ICU admission. Only the predictive value of APTEM CFT and APTEM alpha at baseline for postoperative blood loss were significantly higher ( $p=0.05$ and $p=0.01$ respectively) than fibrinogen (Table 5$)$.

\section{Discussion}

In this study we demonstrated that plasma fibrinogen levels and EXTEM alpha, INTEM $\mathrm{CT}$, FIBTEM CT/MCF, and APTEM CT outside the reference range in the first hours postoperatively were associated with increased bleeding. When analyzing the ability to predict blood loss, all ROTEM variables except CT parameters were associated with a moderate predictive value. Only APTEM CFT and alpha had a significantly higher predictive ability than classical fibrinogen determination by the Clauss method.

As expected, all hemostatic variables changed significantly from baseline to directly after surgery. In the current study median platelet count decreased by $35 \%$ and 
Table 3: Comparison of median blood loss (IQR) in the total patient population with routine laboratory and ROTEM values outside and within the reference range at $\mathrm{T2}$.

\begin{tabular}{|c|c|c|c|c|c|c|c|c|}
\hline & & \multirow{2}{*}{$\begin{array}{c}\mathrm{N} \\
\mathrm{G} 1\end{array}$} & \multirow{2}{*}{$\begin{array}{c}\mathrm{N} \\
\mathrm{G} 2\end{array}$} & \multicolumn{2}{|c|}{$\begin{array}{l}\text { Value hemostatic } \\
\text { parameter } \\
\text { Median (IQR) }\end{array}$} & \multicolumn{2}{|c|}{$\begin{array}{l}\text { Blood loss } \\
\text { Median (IQR) }\end{array}$} & \multirow{2}{*}{$\begin{array}{l}\text { Comp. of } \\
\text { blood loss }\end{array}$} \\
\hline & & & & G1 & G2 & G1 & $\mathrm{G} 2$ & \\
\hline \multicolumn{2}{|c|}{ Hemoglobin ( $\mathrm{mmol} / \mathrm{L})$} & 1 & 96 & $\begin{array}{l}8.7 \\
(8.7-8.7)\end{array}$ & $\begin{array}{l}6.3 \\
(5.9-6.8)\end{array}$ & $\begin{array}{l}500 \\
(500-500)\end{array}$ & $\begin{array}{l}755 \\
(560-1098)\end{array}$ & 0.330 \\
\hline \multicolumn{2}{|c|}{ Hematocrit (L/L) } & 0 & 97 & - & $\begin{array}{l}30 \\
(29-33)\end{array}$ & - & $\begin{array}{l}730 \\
(545-1095)\end{array}$ & - \\
\hline \multicolumn{2}{|c|}{ Platelet count $\left(10^{9} / \mathrm{L}\right)$} & 44 & 53 & $\begin{array}{l}154 \\
(139-168)\end{array}$ & $\begin{array}{l}105 \\
(87-119)\end{array}$ & $\begin{array}{l}660 \\
(515-1168)\end{array}$ & $\begin{array}{l}810 \\
(588-1075)\end{array}$ & 0.386 \\
\hline \multicolumn{2}{|c|}{ Fibrinogen $(\mathrm{g} / \mathrm{L})$} & 48 & 46 & $\begin{array}{l}1.9 \\
(1.8-2.4)\end{array}$ & $\begin{array}{l}1.5 \\
(1.4-1.6)\end{array}$ & $\begin{array}{l}600 \\
(500-874)\end{array}$ & $\begin{array}{l}890 \\
(673-1298)\end{array}$ & $<0.001$ \\
\hline \multirow[t]{4}{*}{ EXTEM } & $\mathrm{CT}(\mathrm{s})$ & 62 & 34 & $\begin{array}{l}53 \\
(48-57)\end{array}$ & $\begin{array}{l}68 \\
(65-75)\end{array}$ & $\begin{array}{l}685 \\
(553-1023)\end{array}$ & $\begin{array}{l}865 \\
(528-1200)\end{array}$ & 0.292 \\
\hline & $\mathrm{CFT}(\mathrm{s})$ & 63 & 34 & $\begin{array}{l}125 \\
(108-135)\end{array}$ & $\begin{array}{l}187 \\
(168-204)\end{array}$ & $\begin{array}{l}680 \\
(510-1060)\end{array}$ & $\begin{array}{l}820 \\
(644-1260)\end{array}$ & 0.091 \\
\hline & Alpha $\left({ }^{\circ}\right)$ & 67 & 30 & $\begin{array}{l}65 \\
(63-68)\end{array}$ & $\begin{array}{l}56 \\
(54-57)\end{array}$ & $\begin{array}{l}670 \\
(510-1030)\end{array}$ & $\begin{array}{l}915 \\
(673-1260)\end{array}$ & 0.019 \\
\hline & $\mathrm{MCF}(\mathrm{mm})$ & 87 & 10 & $\begin{array}{l}57 \\
(52-60)\end{array}$ & $\begin{array}{l}44 \\
(37-46)\end{array}$ & $\begin{array}{l}710 \\
(520-1090)\end{array}$ & $\begin{array}{l}935 \\
(710-1185)\end{array}$ & 0.120 \\
\hline \multirow[t]{4}{*}{ INTEM } & $\mathrm{CT}(\mathrm{s})$ & 68 & 28 & $\begin{array}{l}160 \\
(151-178)\end{array}$ & $\begin{array}{l}211 \\
(199-255)\end{array}$ & $\begin{array}{l}720 \\
(530-1045)\end{array}$ & $\begin{array}{l}2155 \\
(780-3755)\end{array}$ & 0.038 \\
\hline & $\mathrm{CFT}(\mathrm{s})$ & 59 & 38 & $\begin{array}{l}101 \\
(88-110)\end{array}$ & $\begin{array}{l}165 \\
(146-187)\end{array}$ & $\begin{array}{l}650 \\
(500-1060)\end{array}$ & $\begin{array}{l}850 \\
(644-1185)\end{array}$ & 0.055 \\
\hline & Alpha $\left({ }^{\circ}\right)$ & 63 & 34 & $\begin{array}{l}71 \\
(69-73)\end{array}$ & $\begin{array}{l}59 \\
(58-63)\end{array}$ & $\begin{array}{l}670 \\
(510-1090)\end{array}$ & $\begin{array}{l}820 \\
(644-1155)\end{array}$ & 0.149 \\
\hline & $\mathrm{MCF}(\mathrm{mm})$ & 88 & 9 & $\begin{array}{l}54 \\
(50-58)\end{array}$ & $\begin{array}{l}44 \\
(40-45)\end{array}$ & $\begin{array}{l}710 \\
(530-1098)\end{array}$ & $\begin{array}{l}920 \\
(685-1065)\end{array}$ & 0.268 \\
\hline \multirow[t]{2}{*}{ FIBTEM } & $\mathrm{CT}(\mathrm{s})$ & 68 & 28 & $\begin{array}{l}50 \\
(46-52)\end{array}$ & $\begin{array}{l}67 \\
(61-79)\end{array}$ & $\begin{array}{l}680 \\
(513-1015)\end{array}$ & $\begin{array}{l}905 \\
(618-1320)\end{array}$ & 0.030 \\
\hline & $\mathrm{MCF}(\mathrm{mm})$ & 85 & 10 & $\begin{array}{l}9.0 \\
(7.5-12.0)\end{array}$ & $\begin{array}{l}4.5 \\
(3.0-5.0)\end{array}$ & $\begin{array}{l}710 \\
(525-1025)\end{array}$ & $\begin{array}{l}1200 \\
(873-1355)\end{array}$ & 0.016 \\
\hline \multirow[t]{4}{*}{ APTEM } & $\mathrm{CT}(\mathrm{s})$ & 76 & 19 & $\begin{array}{l}57 \\
(52-61)\end{array}$ & $\begin{array}{l}77 \\
(70-89)\end{array}$ & $\begin{array}{l}695 \\
(513-1015)\end{array}$ & $\begin{array}{l}950 \\
(670-1350)\end{array}$ & 0.026 \\
\hline & CFT (s) & 75 & 21 & $\begin{array}{l}128 \\
(115-144)\end{array}$ & $\begin{array}{l}206 \\
(196-234)\end{array}$ & $\begin{array}{l}710 \\
(530-1090)\end{array}$ & $\begin{array}{l}920 \\
(593-1160)\end{array}$ & 0.251 \\
\hline & Alpha $\left({ }^{\circ}\right)$ & 69 & 27 & $\begin{array}{l}65 \\
(63-69)\end{array}$ & $\begin{array}{l}54 \\
(52-57)\end{array}$ & $\begin{array}{l}700 \\
(520-1095)\end{array}$ & $\begin{array}{l}810 \\
(590-1150)\end{array}$ & 0.297 \\
\hline & $\mathrm{MCF}(\mathrm{mm})$ & 87 & 9 & $\begin{array}{l}55 \\
(52-59)\end{array}$ & $\begin{array}{l}43 \\
(36-45)\end{array}$ & $\begin{array}{l}700 \\
(520-1090)\end{array}$ & $\begin{array}{l}950 \\
(765-1200)\end{array}$ & 0.070 \\
\hline
\end{tabular}

Group 1 (G1): patients with values within the reference range; Group 2 (G2): patients with values beyond the reference range; $C T=$ clotting time; $C F T=c l o t$ formation time; $M C F=$ maximal clot firmness; IQR=interquartile range 


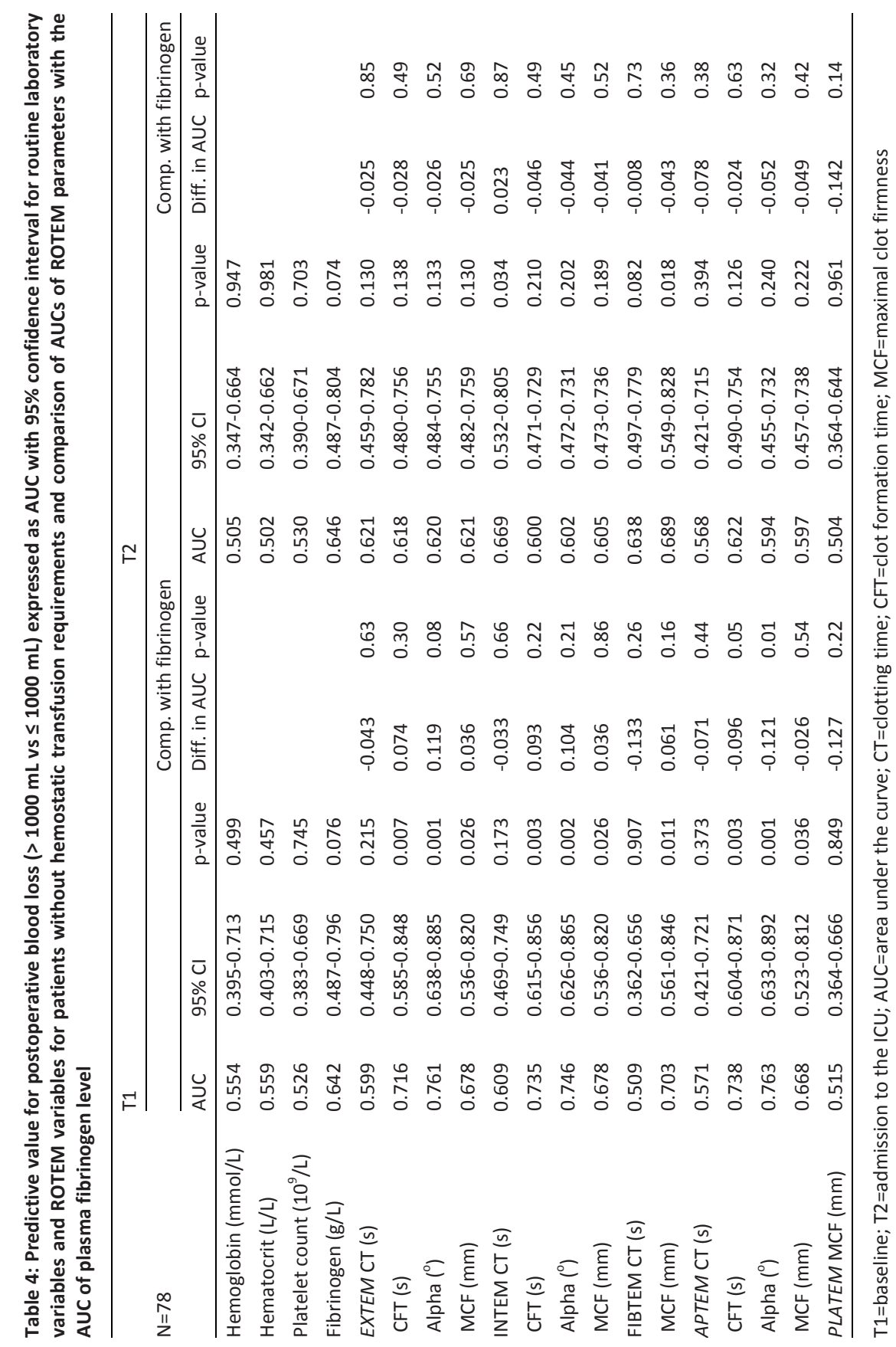


fibrinogen levels by $42 \%$ in the first hour after surgery. The most important causes of reduction in hemostatic factors are hemodilution [19], consumptive coagulopathy [20], and excessive fibrinolysis [21, 22]. Although fibrinolysis was not estimated directly, it likely does not play a significant role in our patients, since the results of the APTEM assay were not different from the results of the EXTEM assay at the different time points, indicating no hyperfibrinolysis. This could possibly be explained by the fact that all patients received tranexamic acid during surgery.

It would be expected that changes induced by CPB, like reductions in platelet count and fibrinogen, will be reflected by the ROTEM assay. The decrease in FIBTEM MCF is comparable with the decrease in fibrinogen at ICU admission. This is in line with the excellent correlations between the two variables $[5,6]$. This suggests that FIBTEM MCF could be an accurate ROTEM parameter for rapid assessment of fibrinogen levels. The reason for a greater change in FIBTEM MCF than the MCFs in other ROTEM assays, could be related to compensatory interactions between platelets, red blood cells and coagulation factors in the other assays. Since fibrinogen is a cofactor that enhances platelet aggregation [23], MCF values may not decrease extensively if platelet counts are within the normal range $[15,24]$. The median platelet count in our study directly postoperatively was just below the normal range. However, platelet count seems to be high enough to compensate for a decreased fibrinogen level compared to baseline in ROTEM assays other than FIBTEM with regard to the MCF parameter. The decrease in platelet count, should be reflected in PLATEM. This was, however, the only variable that did not change after CPB. CPB would also lead to platelet dysfunction [25], but platelet dysfunction cannot be detected with ROTEM tests because of the strong activation of platelets [6]. According to our results we conclude that PLATEM MCF does not reflect the contribution of platelets. The role of platelets can probably better be assessed in tests describing the function of platelets, and not by ROTEM.

The specific contribution of fibrinogen and platelets to dilutional coagulopathy remains controversial. While Schols et al. observed that platelets partially compensate to fibrin clot formation measured by thromboelastography in conditions of plasma dilution in vitro [26], others [27] demonstrated in patients subjected to in vivo dilution that platelets were indeed important in fibrin clot formation, in a fibrinogen independent way. In contrast, Lang et al. [15] concluded in an in vitro study that the clot strength increases in a fibrinogen concentration-dependent manner independent of platelet count, even in case of thrombocytopenia.

According to our predefined analysis, patients having fibrinogen levels below the reference range had a significantly higher median total blood loss than other patients, whereas platelet count was not significantly associated with blood loss. Karlsson et al. also found that low fibrinogen levels, even within the normal range, are an important cause of coagulopathy after cardiac surgery with CPB [28]. Concerning the fibrinogen 
dependent ROTEM assay, FIBTEM parameters outside the reference range were also related to higher blood loss, suggesting a major role for fibrinogen in clot formation and/or stability. However, the importance of fibrinogen did not clearly emerge when assessing the capacity of the different tests discriminating between patients with blood loss of less or more than $1000 \mathrm{~mL}$ with ROC analysis. In general, all ROTEM tests except CT have moderate ability to predict blood loss with higher predictive abilities for postoperative bleeding than the predictive ability of plasma fibrinogen level (showing the highest AUC of the routine laboratory parameters), mainly at baseline. However, only the differences in AUC of APTEM CFT and APTEM alpha angle compared to fibrinogen reached statistical significance. FIBTEM MCF at ICU admission demonstrated a higher predictive ability for blood loss than the other tests, but this was not significantly different from plasma fibrinogen level. Lee et al. [13] did also investigate ROTEM's predictive value for chest tube drainage after cardiac surgery with CPB in three hundred twenty-one patients. From this study it was concluded that ROTEM did not substantially improve prediction of chest tube drainage, beyond routine clinical and laboratory variables, although some ROTEM variables were independently associated with chest tube drainage post CPB. Our results confirm the results of Lee et al. that despite of the poor predictive value for blood loss, ROTEM results are not inferior to fibrinogen in predicting relevant blood loss. That ROTEM is only moderately associated with the prediction of blood loss, is not surprising given the numerous factors related to blood loss in cardiac surgical patients, like surgical bleeding, acidosis, CPB time and body weight $[13,29]$. However, the advantage of ROTEM compared to plasma fibrinogen levels, is that the test has shorter turnaround times, it gives information about the complete hemostatic profile of the patient and it could therefore be a faster method to guide hemostatic therapy.

This study had limited sample size, but was large enough to detect statistically significant AUCs for various ROTEM parameters. However, the power was too low to detect an increase in AUC by at least 0.05 when comparing the discriminative ability (AUC) between ROTEM parameters and fibrinogen. Only AUC differences in the order of 0.1 or larger could be detected with statistical significance. For example the AUC difference between APTEM CFT versus fibrinogen was 0.096 associated with a $p$-value of 0.05 . Another limitation is that the red blood cell content of the fluid in the mediastinal drains was not measured, which might be different between patients because of progression from blood to serosanguineous drainage.

In summary, results of routine laboratory tests and the ROTEM assay outside the reference range are associated with higher median blood loss when compared with values within the reference range. ROTEM parameters have moderately high predictive abilities for postoperative blood loss, and are slightly higher than the predictive ability of plasma fibrinogen level. The results of this study propose a role for ROTEM as 
a point-of-care method to monitor hemostasis in cardiac surgical patients, because of a higher predictive ability for blood loss and a more complete reflection of hemostasis compared to fibrinogen. Besides this, ROTEM is a faster method to guide hemostatic therapy. 


\section{References}

1. Christensen, M.C., et al., Costs of excessive postoperative hemorrhage in cardiac surgery. J Thorac Cardiovasc Surg, 2009. 138(3): p. 687-93.

2. Woodman, R.C. and L.A. Harker, Bleeding complications associated with cardiopulmonary bypass. Blood, 1990. 76(9): p. 1680-97.

3. Despotis, G.J. and L.T. Goodnough, Management approaches to platelet-related microvascular bleeding in cardiothoracic surgery. Ann Thorac Surg, 2000. 70(2 Suppl): p. S20-32.

4. Solomon, C., N. Rahe-Meyer, and B. Sorensen, Fibrin formation is more impaired than thrombin generation and platelets immediately following cardiac surgery. Thromb Res, 2011. 128(3): p. $277-82$.

5. Ogawa, S., et al., A comparative evaluation of rotation thromboelastometry and standard coagulation tests in hemodilution-induced coagulation changes after cardiac surgery. Transfusion, 2012. 52(1): p. 14-22.

6. Olde Engberink, R.H., et al., Rapid and correct prediction of thrombocytopenia and hypofibrinogenemia with rotational thromboelastometry in cardiac surgery. J Cardiothorac Vasc Anesth, 2014. 28(2): p. 210-6.

7. Wasowicz, M., et al., The incremental value of thrombelastography for prediction of excessive blood loss after cardiac surgery: an observational study. Anesth Analg, 2010. 111(2): p. 331-8.

8. Anderson, L., et al., An audit of red cell and blood product use after the institution of thromboelastometry in a cardiac intensive care unit. Transfus Med, 2006. 16(1): p. 31-9.

9. Ganter, M.T. and C.K. Hofer, Coagulation monitoring: current techniques and clinical use of viscoelastic point-of-care coagulation devices. Anesth Analg, 2008. 106(5): p. 1366-75.

10. Reinhofer, M., et al., The value of rotation thromboelastometry to monitor disturbed perioperative haemostasis and bleeding risk in patients with cardiopulmonary bypass. Blood Coagul Fibrinolysis, 2008. 19(3): p. 212-9.

11. Davidson, S.J., et al., Can ROTEM thromboelastometry predict postoperative bleeding after cardiac surgery? J Cardiothorac Vasc Anesth, 2008. 22(5): p. 655-61.

12. Ghavidel, A.A., et al., Rotational thromboelastometry in prediction of bleeding after cardiac surgery. Asian Cardiovasc Thorac Ann, 2015.

13. Lee, G.C., et al., Does rotational thromboelastometry (ROTEM) improve prediction of bleeding after cardiac surgery? Anesth Analg, 2012. 115(3): p. 499-506.

14. Petricevic, M., et al., Activated coagulation time vs. intrinsically activated modified rotational thromboelastometry in assessment of hemostatic disturbances and blood loss after protamine administration in elective cardiac surgery: analysis from the clinical trial (NCT01281397). J Cardiothorac Surg, 2014. 9: p. 129.

15. Lang, T., et al., The effects of fibrinogen levels on thromboelastometric variables in the presence of thrombocytopenia. Anesth Analg, 2009. 108(3): p. 751-8.

16. Henskens, Y.L., M. Beckers. E., Implementatie van de trombo-elastografie (ROTEM $\left.{ }^{\circledR}\right)$ in het MUMC+: (On)mogelijkheden van laboratoriumtesten bij bloedverlies en verworven hemostase afwijkingen. Tijdschr Bloedtransfusie, 2012. 5: p. 3-13.

17. Bevan, D.H., Cardiac bypass haemostasis: putting blood through the mill. Br J Haematol, 1999. 104(2): p. 208-19.

18. Coakley, M., et al., Assessment of thrombin generation measured before and after cardiopulmonary bypass surgery and its association with postoperative bleeding. J Thromb Haemost, 2011. 9(2): p. 28292.

19. Chandler, W.L., Effects of hemodilution, blood loss, and consumption on hemostatic factor levels during cardiopulmonary bypass. J Cardiothorac Vasc Anesth, 2005. 19(4): p. 459-67. 
20. Despotis, G.J., M.S. Avidan, and C.W. Hogue, Jr., Mechanisms and attenuation of hemostatic activation during extracorporeal circulation. Ann Thorac Surg, 2001. 72(5): p. S1821-31.

21. Chandler, W.L. and T. Velan, Plasmin generation and D-dimer formation during cardiopulmonary bypass. Blood Coagul Fibrinolysis, 2004. 15(7): p. 583-91.

22. Khalil, P.N., et al., Activation of fibrinolysis in the pericardial cavity after cardiopulmonary bypass. Thromb Haemost, 2004. 92(3): p. 568-74.

23. Karkouti, K., et al., The relationship between fibrinogen levels after cardiopulmonary bypass and large volume red cell transfusion in cardiac surgery: an observational study. Anesth Analg, 2013. 117(1): p. 14-22.

24. Nagler, M., et al., Impact of changes in haematocrit level and platelet count on thromboelastometry parameters. Thromb Res, 2013. 131(3): p. 249-53.

25. Johansson, P.I., et al., Coagulopathy and hemostatic monitoring in cardiac surgery: an update. Scand Cardiovasc J, 2012. 46(4): p. 194-202.

26. Schols, S.E., et al., Effects of plasma dilution on tissue-factor-induced thrombin generation and thromboelastography: partly compensating role of platelets. Transfusion, 2008. 48(11): p. 2384-94.

27. Ninivaggi, M., et al., Additive roles of platelets and fibrinogen in whole-blood fibrin clot formation upon dilution as assessed by thromboelastometry. Thromb Haemost, 2014. 111(3): p. 447-57.

28. Karlsson, M., et al., Plasma fibrinogen level, bleeding, and transfusion after on-pump coronary artery bypass grafting surgery: a prospective observational study. Transfusion, 2008. 48(10): p. 2152-8.

29. Nakayama, Y., et al., Thromboelastometry-guided intraoperative haemostatic management reduces bleeding and red cell transfusion after paediatric cardiac surgery. Br J Anaesth, 2014. 

CHAPTER 8

GENERAL DISCUSSION 

Managing of (anti)-coagulation in cardiac surgical patients undergoing cardiopulmonary bypass ( $\mathrm{CPB}$ ) is challenging, especially when engaging corrective interventions. The choice of the right monitoring systems is therefore crucial for clinical decisionmaking. In this thesis the activated clotting time (ACT) test, which is routinely used during $C P B$, is discussed, and a possible role for the calibrated automated thrombography (CAT) assay and the rotational thromboelastometry (ROTEM) test in (anti)coagulation monitoring and prediction of blood loss is proposed.

Activation of the coagulation system in cardiac surgery with CPB is part of a "whole body inflammatory response". This response is caused by tissue injury, contact of blood with the surfaces of the extracorporeal circuit and the transfusion of shed pericardial blood. As a consequence, thrombin is continually produced by the extrinsic coagulation pathway at sites of injury. Small amounts of thrombin activate platelets, coagulation factors of both the extrinsic and intrinsic coagulation pathways, and the fibrinolytic system [1]. This results in consumption of coagulation factors, contributing to postoperative bleeding [2].

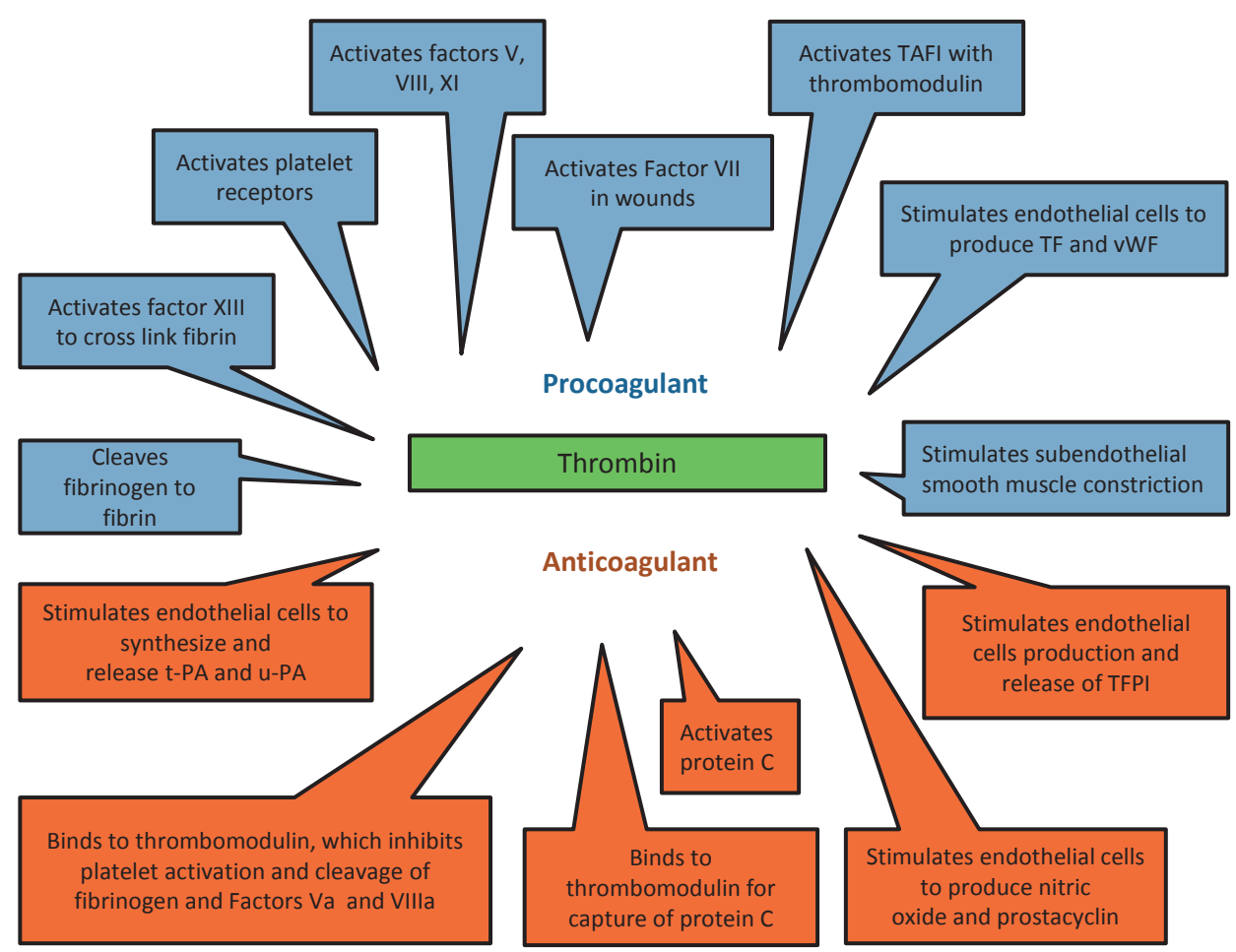

Figure 1: Thrombin and its central role in the hemostatic system 
The whole thrombin generation (TG) process is influenced by all the thrombindependent regulation loops of all coagulation phases (Figure 1) [3]. Furthermore, platelets play an important role in TG by providing a procoagulant membrane surface, and hence supporting the formation of more thrombin [4]. Because of the continuous activation of the clotting system, systemic anticoagulation using heparin is a conditio sine qua non for CPB during cardiac surgery. However, heparin can minimize excessive CPB-related activation of the hemostatic system, but it fails to inhibit TG completely [1].

The working mechanism of heparin is to accelerate the natural inhibitor of coagulation, i.e. antithrombin (AT). When heparin responsiveness is impaired, this is often attributed to AT deficiency [2, 5-7]. To gauge a patient's heparin responsiveness, meaning the effect of heparin on the anticoagulation level, the ACT is most frequently used in cardiac surgery. Either a low heparin level or a low AT level might explain a low or decreased ACT value. To verify this assumption we determined the relationship between AT and heparin sensitivity index (HSI) before the establishment of CPB (Chapter 3). The results of this observational study indicated that decreased heparin responsiveness, measured by ACT, was not associated with subnormal AT activity. All patients with subnormal AT activity (55\%-79\%) showed adequate ACT values. Apparently a clear association between HSI and AT activity is lacking. It has previously been reported in an in vitro study that excessive drops in AT would increase TG significantly [8], which underlines the crucial role of AT in hemostasis and anticoagulation. The lack of association between AT on one hand, and HSI or ACT on the other hand, suggests that heparin monitoring by ACT assay seems to be unreliable. This can be explained by the fact that the ACT assay only reflects the initiation phase and not the total course of the coagulation cascade. In Figure 2A the initiation phase of coagulation is presented, showing that fibrin formation can already occur when there are only minute amounts of thrombin formed. The ACT test will end after the first traces of thrombin are detected $[9,10]$. This will have minimal influence on the level of AT in this phase, even if the AT level is reduced with $50 \%$, and will therefore not affect the ACT. The small amounts of thrombin formed in the initiation phase amplify the procoagulant response by activating coagulation factors and platelets (Figure 2B). In the propagation phase (Figure $2 \mathrm{C}$ ) a burst of TG occurs, and this will require a large amount of AT [11]. Comparing Figure $2 \mathrm{~A}$ with Figure $2 \mathrm{C}$, it is clear that the ratio between thrombin and $\mathrm{AT}$ is reversing when coagulation continues: the more thrombin is formed, the less AT is left. If a patients' AT level is low, it will affect a test, in this phase of coagulation, since the contribution of AT is relatively more important at this moment than in the initiation phase. This clarifies why it is nearly impossible to associate AT deficiency with the ACT. 


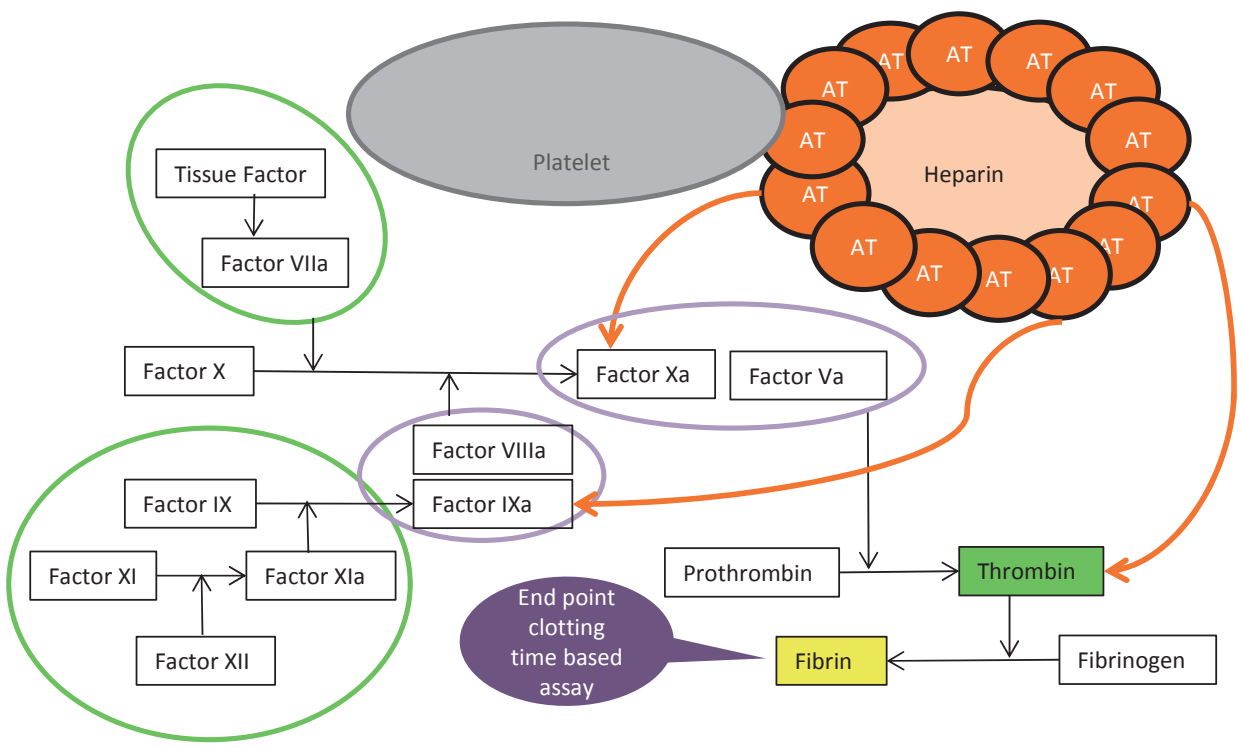

Figure 2A: Initiation phase

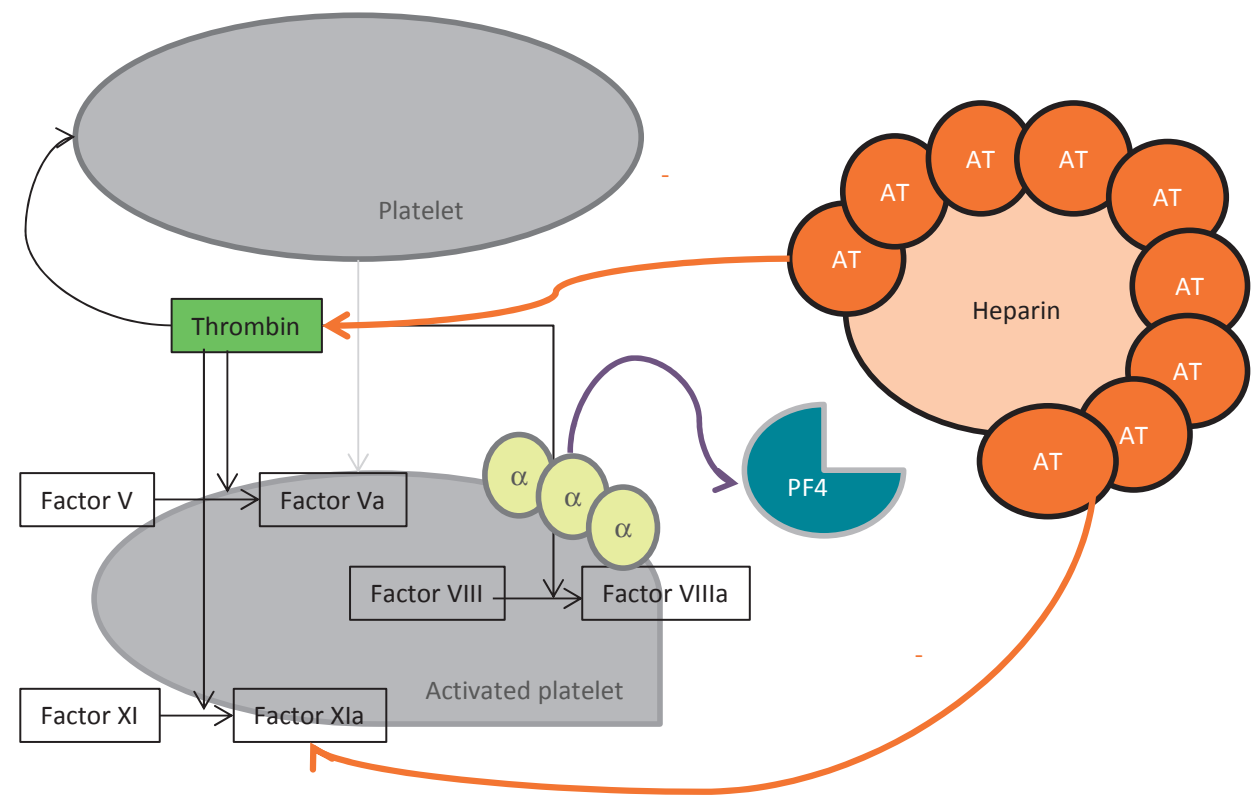

Figure 2B: Amplification phase 


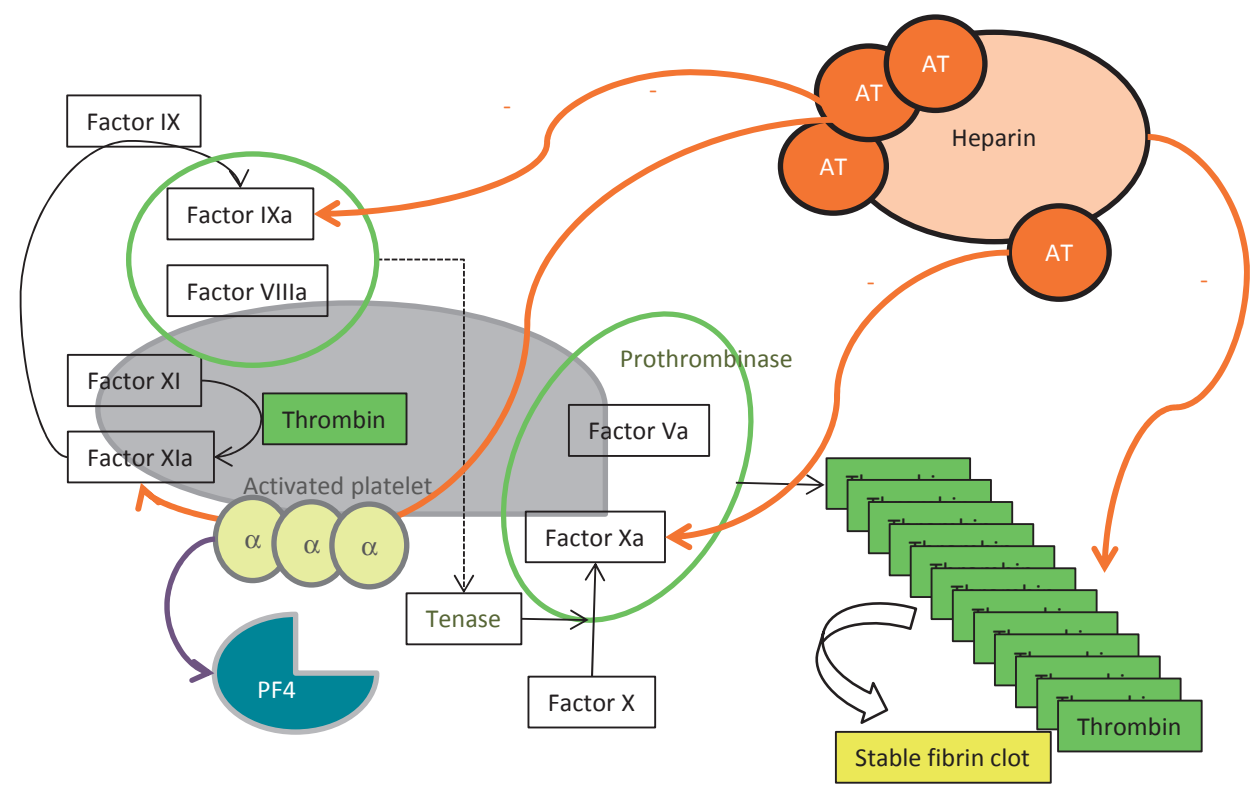

Figure 2C: Propagation phase

Furthermore, it was demonstrated (Chapter 3) that high platelet counts are associated with decreased heparin responsiveness. This can be explained not only by the neutralizing effect of platelet factor 4 on heparin, but also by binding of heparin to platelets themselves, activated and non-activated [12]. The concentration of heparin required to inhibit or delay coagulation is directly related to the number of platelets. The role of the platelet is to act as a surface on which coagulation can take place. Right from the very first beginning platelets and thrombin generation cooperate [13]: small amounts of thrombin, formed in the initiation phase, will activate platelets (Figure 2A-C). This can result in further binding and neutralizing of heparin. Although, this neutralizing effect will become stronger in the amplification and propagation phase of coagulation when more and more platelets become activated. It can be concluded that a failure to achieve an adequate ACT usually is not an indicator of AT deficiency, which is presumed to be of significant influence, but could be affected by high platelet counts.

Besides the influence of hemostatic factors, like AT level and platelet count, the ACT is also influenced by the technical performance of the type of ACT device. Bias between different ACT monitors may have a significant impact on heparin dosage [14]. Characteristics of specific compounds used to activate the coagulation may play a crucial role. In Chapter 4 we concluded that ACT measurements depend on the analyzer that is used. Although the reproducibility of analyzers seems to be good, a clear agreement between analyzers is lacking. An ACT value measured with a specific device does not imply the same level of anticoagulation, when measured with another device. 
It is therefore advisable to evaluate the performance of a new ACT device in every specific clinic in accordance with the local anticoagulation protocols. Additionally, the lack of agreement between analyzers needs to be considered when interpreting existing literature.

Obviously, there is a need for superior whole blood tests, reflecting the anticoagulant status and the hemostatic capacity of the patients' blood. Measuring TG by calibrated automated thrombography (CAT), could be such a test. Thrombin generation in PRP reflects the major part of the physiological clotting system, including the interaction between platelets and the clotting system. In order to reveal the ultimate test reflecting all kind of disturbances in coagulation related to CPB, we measured the hemostatic capacity with several coagulation tests (CAT, conventional clotting tests and tissue factor pathway inhibitor) (Chapter 5). Time points of blood sample collection were representative for changes in the hemostatic equilibrium and test results should provide more information on the responses to such changes. Both ACT and CAT measurements demonstrated the anticoagulant effect of heparin before the start of CPB. CPB leads to an activation of the coagulation system, which was clearly reflected by the parameters of the CAT. After reaching its lowest value as a result of heparinization, the mean levels of the endogenous thrombin potential elevated again during CPB, and both lag time and time to peak decreased. In contrast, the mean level of the D-dimers showed the opposite at the start of CPB, indicating that this parameter does not reflect the activation of the coagulation system. In the same way as in Chapter 3, the ACT was not correlated with the level of heparinization. Immediately after the start of CPB, the ACT increased, despite of decreasing levels of heparin and AT. After this initial increase, ACT values decreased during CPB, while anti-Xa and AT levels remain stable. After heparin neutralization, CAT parameters tended to normalize, but were still different from baseline. This was probably caused by the effect of the tissue factor pathway inhibitor (TFPI), which was considerably higher after protamine administration than at baseline. TFPI is mobilized from vascular endothelium to the circulation by heparin [15]. Our results showed that after heparin neutralization, TFPI levels were significantly higher than baseline levels, and as a consequence all clotting time based tests (ACT, PT and aPTT) were still prolonged compared to baseline. Besides the influence of TFPI, the ACT test could be prolonged by a significantly lower platelet count or fibrinogen level compared to baseline. After all, clot formation in whole blood represents the interaction of plasma coagulation components, like fibrinogen, platelets, and red blood cells [16] (see Figure 2A). According to the CAT results after heparin neutralization, platelets and fibrinogen probably play a minor role. Firstly, TG capacity is clearly reduced in platelet poor conditions. Furthermore, the outcome of the TG test is moderately influenced by the decrease in fibrinogen [17]. In addition, anti-Xa measurements suggest that heparin was almost fully neutralized. Therefore, TFPI could be the 
reason for ongoing anticoagulation and the influence of this factor could be underestimated. The importance of this study was that TG could be determined during CPB despite the high level of heparin. The disadvantage of the CAT assay used in the study, is the measurement in plasma. Determinations of TG in whole blood would be beneficial as the contribution of all blood cells will be determined, no centrifugal step is needed and turnaround-times can be significantly shorter.

Thromboelastography (TEG) and rotational thromboelastometry (ROTEM) performed in whole blood evaluate the hemostatic system globally and are used to guide the management of significant postoperative bleeding. In Chapter 7 it was investigated how routine coagulation tests and ROTEM variables change after CPB. It was shown that all variables were significantly changed from baseline directly postoperative but to different degrees. Furthermore, the results of the ROTEM assay depend on the phase of coagulation (Figure $2 \mathrm{~A}-\mathrm{C}$ ) represented by the different ROTEM variables $[18,19]$ and the activator that is used (triggering the extrinsic or intrinsic pathway). It was obvious that the contribution of platelets was not visible in PLATEM. One could expect a decrease in PLATEM after CPB, since both platelet count and platelet function are decreased. However, PLATEM remained at the same level as baseline. Maximal fibrinogen-dependent clot strength (FIBTEM) showed equal decreases as fibrinogen levels. It should be underlined that FIBTEM is measured in whole blood and fibrinogen levels in plasma, which could have influenced the result. First, coagulation measured in whole blood is more affected by concomitant coagulation factor activities [20] and second, the Clauss method should overestimate fibrinogen levels after dilution with colloids in vitro due to clouding of the plasma, disturbing the optical test method [21, 22].

It would be extremely useful to have a test that accurately predicts an individual's coagulation potential and that may improve the management of significant postoperative bleeding. Blood loss is a major clinical consequence after cardiac surgery with CPB. To prevent this bleeding risk as much as possible, it would be beneficial to assess the hemostatic condition of a patient, pre-, peri- and postoperatively. Measuring the TG capacity of blood ex vivo should be a reliable parameter in hemostatic and thrombotic diseases. When the endogenous thrombin potential (ETP) of TG is below $20 \%$ of normal, a bleeding tendency can occur $[3,23,24]$. In Chapter 6 we investigated the role of CAT and routine laboratory tests in the prediction of blood loss in CPB patients at two time points: T1) pre-bypass before heparin administration; and, T2) $5 \mathrm{~min}$ after protamine administration. T1 measurements would give information about the predictive value of the parameters before the start of CPB suggesting differences in baseline hemostatic capacity between the patients, whereas T2 measurements may give a prediction of blood loss after CPB including the influence of all hemostatic disturbances related to CPB. The only laboratory variable measured pre-CPB predicting blood loss after surgery was platelet count. Post-CPB, both platelet count and fibrinogen were 
significantly predictive for blood loss. However, it is remarkable that fibrinogen was weaker associated with blood loss than platelet count. Fibrinogen is the first coagulation factor to fall below a critical low level upon dilution $[25,26]$, and expected to be related to blood loss. Platelet dysfunction is considered to be the most important defect in hemostasis in the early postoperative period after CPB [27], and is also associated with blood loss [28]. Conversely, platelet count showed, not to be decisive in its ability to identify patients with excessive blood loss after cardiac surgery [27]. However, measurement of the thrombin generating capacity both pre- and postoperatively, correlates well with the clinically observed bleeding tendency postoperatively in cardiac surgical patients. Apparently, our results strengthen the notion that it is not the initiation phase of clotting (Figure 2A) but the propagation phase (Figure 2C) that determines hemostasis and blood loss after cardiac surgery. Calibrated automated thrombin generation, determined by simultaneous prothrombin activation and thrombin inactivation [29], measures the remaining capacity of blood to generate a thrombin burst, indicating an increased risk of thrombosis or bleeding [9]. The key finding of this study was that TG capacity measured by the CAT assay performed preoperatively, provides predictive information for non-surgical blood loss after cardiac surgery. The CAT assay, including the contribution of platelet function [30] and fibrinogen [31], might be considered as a standard screening test to assess the hemostatic condition preoperatively.

In Chapter 5 we investigated whether TG measured by CAT intraoperatively was associated with an increased risk of bleeding after surgery. TG parameters were measured before and after the start of CPB, and were associated with postoperative blood loss. TG was found to predict bleeding tendency when performed intraoperatively and particularly in the presence of platelets (PRP). Again, it was clear that the overall performance of the peak height and ETP (propagation phase) was much better in predicting blood loss after surgery than the lag time (initiation phase) and time to peak. This is in agreement with the observation that the ACT has a poor predictive value.

Besides the predictive value of CAT, it is also interesting to investigate how the routine coagulation tests and ROTEM variables are related to postoperative blood loss, particularly when values fall outside the reference range (Chapter 7). Not every test result outside the reference range, can be associated with increased bleeding risk. In this study it was observed that plasma fibrinogen and ROTEM parameters outside the reference range were related to higher blood loss. This seems to be of crucial importance in detecting a patient at increased bleeding risk. However, the accuracy of laboratory tests and ROTEM to discriminate patients with high blood loss from patients with low blood loss corrected for hemostatic blood product requirements, determined at baseline and directly postoperative, was only moderate. 
It can be concluded that the results of the routine laboratory tests and the ROTEM assay beyond the reference range are associated with higher median blood loss when compared with values within the reference range. The performance of ROTEM parameters is better than fibrinogen level in predicting blood loss only when APTEM CFT and alpha are considered at baseline. FIBTEM MCF at ICU admission demonstrated a higher predictive ability for blood loss than the other tests, but this was not significantly different from plasma fibrinogen level. In other words, APTEM CFT and APTEM alpha measured at baseline are the only ROTEM parameters with a shorter turnaround time, that are more clinically useful than plasma fibrinogen level.

The disadvantage of viscoelastic assays is that clot formation is only one component of the effects of thrombin formation, and not directly related to hemostasis [32]. Furthermore, clotting time dependent ROTEM parameters are probably not clinically useful as these tests reflect only the initiation phase of coagulation (Figure $2 \mathrm{~A}$ ). And in addition, according to our results, the reflection of platelets by ROTEM is not clearly recognizable.

In general, viscoelastic tests have some important diagnostic limitations. Although they are determined in whole blood, such methods provide by no means a complete picture of the clotting system, as well as don't take into account the influence of flow dynamics. Moreover, as the direct addition of an activator automatically by-passes primary hemostasis, disorders of primary hemostasis cannot be detected. On the other hand, effects of acetyl salicylic acid and adenosine diphosphate-receptor antagonists cannot be determined either [33].

\section{Conclusion and future perspectives}

At present, there are several drawbacks in monitoring the hemostatic condition of the cardiac surgical patient. Activated clotting times are still used worldwide, though test results can be misleading. Although ROTEM measurements were performed in whole blood, this test did not very well identify patients with an increased risk of bleeding. The predictive value of ROTEM for bleeding risk postoperatively was lower than the predictive value of CAT measured in plasma. Besides the predictive value of CAT for blood loss, we demonstrated that disturbances induced by CPB surgery were well reflected in CAT measurements performed pre-, peri- and post CPB. This leads to the conclusion that measuring thrombin generation with the CAT could be a useful tool to implement in the operation theatre for cardiac surgical procedures. In addition, the development of whole-blood CAT assay is on its way, and point-of-care methods are likely becoming feasible in the near future. 


\section{References}

1. Edmunds, L.H., Jr. and R.W. Colman, Thrombin during cardiopulmonary bypass. Ann Thorac Surg, 2006. 82(6): p. 2315-22.

2. Despotis, G.J., et al., Antithrombin III during cardiac surgery: effect on response of activated clotting time to heparin and relationship to markers of hemostatic activation. Anesth Analg, 1997. 85(3): p. 498-506.

3. Al Dieri, R., et al., The thrombogram in rare inherited coagulation disorders: its relation to clinical bleeding. Thromb Haemost, 2002. 88(4): p. 576-82.

4. Heemskerk, J.W., E.M. Bevers, and T. Lindhout, Platelet activation and blood coagulation. Thromb Haemost, 2002. 88(2): p. 186-93.

5. Avidan, M.S., et al., Recombinant human antithrombin III restores heparin responsiveness and decreases activation of coagulation in heparin-resistant patients during cardiopulmonary bypass. J Thorac Cardiovasc Surg, 2005. 130(1): p. 107-113.

6. Garvin, S., et al., Heparin dose response is independent of preoperative antithrombin activity in patients undergoing coronary artery bypass graft surgery using low heparin concentrations. Anesth Analg, 2010. 111(4): p. 856-61.

7. Ranucci, M., et al., Predictors for heparin resistance in patients undergoing coronary artery bypass grafting. Perfusion, 1999. 14(6): p. 437-442.

8. Butenas, S., C. van't Veer, and K.G. Mann, "Normal" thrombin generation. Blood, 1999. 94(7): p. 216978.

9. Hemker, H.C., R. Al Dieri, and S. Beguin, Thrombin generation assays: accruing clinical relevance. Curr Opin Hematol, 2004. 11(3): p. 170-5.

10. Mann, K.G., K. Brummel, and S. Butenas, What is all that thrombin for? J Thromb Haemost, 2003. 1(7): p. 1504-14.

11. Hoffman, M. and D.M. Monroe, Coagulation 2006: a modern view of hemostasis. Hematol Oncol Clin North Am, 2007. 21(1): p. 1-11.

12. Horne, M.K., 3rd and E.S. Chao, Heparin binding to resting and activated platelets. Blood, 1989. 74(1): p. 238-43.

13. Hemker, H.C., et al., Thrombin generation, a function test of the haemostatic-thrombotic system. Thromb Haemost, 2006. 96(5): p. 553-61.

14. Svenmarker, S., et al., Measurement of the activated clotting time during cardiopulmonary bypass: differences between Hemotec ACT and Hemochron Jr apparatus. Perfusion, 2004. 19(5): p. 289-94.

15. Brodin, E., et al., Regulation of thrombin generation by TFPI in plasma without and with heparin. Transl Res, 2009. 153(3): p. 124-31.

16. Levy, J.H. and R.M. Sniecinski, Activated clotting times, heparin responses, and antithrombin: have we been wrong all these years? Anesth Analg, 2010. 111(4): p. 833-5.

17. Schols, S.E., et al., Increased thrombin generation and fibrinogen level after therapeutic plasma transfusion: relation to bleeding. Thromb Haemost, 2008. 99(1): p. 64-70.

18. Tanaka, K.A., et al., Rotational thromboelastometry (ROTEM)-based coagulation management in cardiac surgery and major trauma. J Cardiothorac Vasc Anesth, 2012. 26(6): p. 1083-93.

19. Theusinger, O.M., et al., The influence of laboratory coagulation tests and clotting factor levels on Rotation Thromboelastometry (ROTEM(R)) during major surgery with hemorrhage. Anesth Analg, 2013. 117(2): p. 314-21.

20. Haas, T., et al., Comparison of thromboelastometry (ROTEM(R)) with standard plasmatic coagulation testing in paediatric surgery. Br J Anaesth, 2012. 108(1): p. 36-41.

21. Adam, S., R. Karger, and V. Kretschmer, Influence of different hydroxyethyl starch (HES) formulations on fibrinogen measurement in HES-diluted plasma. Clin Appl Thromb Hemost, 2010. 16(4): p. 454-60. 
22. Fenger-Eriksen, C., et al., Fibrinogen estimates are influenced by methods of measurement and hemodilution with colloid plasma expanders. Transfusion, 2010. 50(12): p. 2571-6.

23. Dargaud, Y., et al., Evaluation of thrombin generating capacity in plasma from patients with haemophilia $A$ and B. Thromb Haemost, 2005. 93(3): p. 475-80.

24. van Veen, J.J., et al., Calibrated automated thrombin generation and modified thromboelastometry in haemophilia A. Thromb Res, 2009. 123(6): p. 895-901.

25. Fenger-Eriksen, C., et al., Fibrinogen substitution improves whole blood clot firmness after dilution with hydroxyethyl starch in bleeding patients undergoing radical cystectomy: a randomized, placebocontrolled clinical trial. J Thromb Haemost, 2009. 7(5): p. 795-802.

26. Mittermayr, M., et al., Hemostatic changes after crystalloid or colloid fluid administration during major orthopedic surgery: the role of fibrinogen administration. Anesth Analg, 2007. 105(4): p. 905-17, table of contents.

27. Despotis, G.J. and L.T. Goodnough, Management approaches to platelet-related microvascular bleeding in cardiothoracic surgery. Ann Thorac Surg, 2000. 70(2 Suppl): p. S20-32.

28. Solomon, C., N. Rahe-Meyer, and B. Sorensen, Fibrin formation is more impaired than thrombin generation and platelets immediately following cardiac surgery. Thromb Res, 2011. 128(3): p. 277-82.

29. Hemker, H.C., et al., Calibrated automated thrombin generation measurement in clotting plasma. Pathophysiol Haemost Thromb, 2003. 33(1): p. 4-15.

30. Wegert, W., et al., Effects of antiplatelet agents on platelet-induced thrombin generation. Int J Clin Pharmacol Ther, 2002. 40(4): p. 135-41.

31. Kremers, R.M., R.J. Wagenvoord, and H.C. Hemker, The effect of fibrin(ogen) on thrombin generation and decay. Thromb Haemost, 2014. 112(3): p. 486-94.

32. Hemker, H.C., et al., The calibrated automated thrombogram (CAT): a universal routine test for hyperand hypocoagulability. Pathophysiol Haemost Thromb, 2002. 32(5-6): p. 249-53.

33. Lang, T. and M. von Depka, [Possibilities and limitations of thrombelastometry/-graphy]. Hamostaseologie, 2006. 26(3 Suppl 1): p. S20-9. 
SUMMARY 

Patients undergoing cardiac surgery with cardiopulmonary bypass are susceptible to disturbances in hemostasis. Blood loss is one of the most encountered consequences, despite of preoperative evaluation by medical history and routine laboratory coagulation testing. Other clinical consequences of the resulting coagulopathy are mediastinal re-exploration, postoperative stroke due to hypercoagulability and increased mortality. Furthermore, intensive care stay is prolonged and hospital costs are increased

Disturbed hemostasis can be attributed to an abnormal thrombin generation (TG), platelet dysfunction and excessive fibrinolysis. Hemodilution causes reduction in the plasma concentration of coagulation factors and platelet counts to decrease to about $50 \%$ of preoperative levels. Consumption is caused by tissue injury, contact activation with the artificial surfaces of the extracorporeal circuit and the transfusion of shed pericardial blood. Systemic anticoagulation with high doses of unfractionated heparin can disturb the hemostatic system even further.

The primary anticoagulant action of heparin appears to result from its binding with antithrombin (AT). Measuring this anticoagulant effect of heparin is complicated because it depends on many variables which determine the patients sensitivity to heparin, such as: 1 ) the availability of AT, 2) the anticoagulation effect of tissue factor pathway inhibitor (TFPI) released by heparin, 3) the binding of heparin to proteins in the bloodstream, 4) the binding of heparin to endothelium, and 5) platelets which are heparin neutralizing themselves but neutralize heparin also by the release of platelet factor 4 (PF4).

All of these aspects are causing defects in the coagulation system, and therefore, it is, challenging to monitor the hemostatic capacity of a cardiac surgical patient in a proper way. At the moment equipment to monitor hemostasis and anticoagulation adequately is lacking. Heparinization is currently monitored by either measuring heparin concentration, or by measuring its effect on clotting times. Between the different heparin concentration tests there is a lack of agreement, and monitoring heparin concentration has not uniformly been associated with blood loss. The most frequently used test based on clotting time, the activated partial thromboplastin time (aPTT), is not suitable during CPB to guide heparin concentrations $>1 \mathrm{IU} / \mathrm{mL}$. Therefore, prolongation of coagulation during cardiac surgery is measured by the so-called activated clotting time (ACT). The endpoint of this whole blood test is formation of fibrin. However, it is surprising that there is no relation between the primary cofactor (AT) of heparin and the ACT.

In Chapter 3 the relationship between AT and heparin sensitivity index (HSI) is determined. The results of this observational study indicated that decreased heparin responsiveness, measured by ACT, was not associated with subnormal AT activity. This suggests that heparin monitoring by ACT assay seems to be unreliable. This can be explained by the fact that the ACT assay only reflects the initiation phase of coagula- 
tion and not the total course of the coagulation cascade. The test will end after only $5 \%$ of the total amount of thrombin is generated, whereas $95 \%$ of the hemostatic capacity is not assessed. Furthermore, it is demonstrated in this chapter that high platelet counts are associated with decreased heparin responsiveness, explained by the neutralizing effect of thrombocytes. The concentration of heparin required to inhibit or delay coagulation is directly related to the number of thrombocytes. From this study it can be concluded that a failure to achieve an adequate ACT usually is not an indicator of AT deficiency, but could be affected by high platelet counts.

The ACT is also influenced by the technical performance of the type of ACT device. ACT analyzers differ by activators, detection systems, and use different sample volumes. Consequently, the ACT values measured by different systems are not comparable. This is also confirmed in Chapter 4, in which the GEM PCL (Instrumentation Laboratory), the Hemochron 801 (International Technidyne Corporation) and the ACT II Automated Coagulation Timer (Medtronic) are compared. Although the reproducibility of analyzers seems to be good, a clear agreement between analyzers is lacking. It is therefore advisable to evaluate the performance of a new ACT device in every specific clinic in accordance with the local anticoagulation protocols.

There is growing evidence suggesting that coagulation is increasingly activated with time during CPB, even with ACT-based protocols. This might lead to a progressive consumptive coagulopathy resulting in a prolonged ACT while heparin levels are decreased. This leads to an incorrect estimation of the anticoagulation status of the patient. Therefore, it would be desirable to have a superior test reflecting the anticoagulation status and the total hemostatic capacity of the patient. Routine laboratory tests (like platelet count, fibrinogen and D-dimers) do not reflect the activity of both coagulation- and anticoagulation proteins, and the interaction with thrombocytes. In addition, tests are often time consuming since these are measured in plasma, and results are not rapidly available, so they do not reflect the current state of the coagulation system. In Chapter $\mathbf{5}$ it is attempted to reveal the ultimate test reflecting all kinds of disturbances in coagulation related to CPB. The Calibrated Automated Thrombography (CAT) assay measuring the total amount of TG in time could be such a test. Besides this test, routine laboratory tests, the ACT and TFPI were also measured. Both ACT and CAT measurements demonstrated the anticoagulant effect of heparin before the start of $\mathrm{CPB}$. Heparinized samples were neutralized with polybrene in order to investigate the underlying thrombin generating capacity. This demonstrated that TG capacity diminishes considerable following heparinization compared to baseline. This could be due to the anticoagulant effect of TFPI. However, during CPB, TG levels continued to increase. This procoagulant effect was well reflected by the CAT measurements. The ACT showed a prolongation after the start of $\mathrm{CPB}$, which does not correspond to the procoagulant effect of CPB and the decrease in AT. During CPB the ACT did not correlate 
anymore with the level of heparinization measured by the anti-Xa assay. Plasma Ddimers decreased after the start of CPB, which neither reflects the procoagulant effect of CPB. This study demonstrates that TG can be determined during CPB despite of the high heparinization level, and that CAT reflects better the hemostatic capacity of the patient than the routine tests. In addition, it was investigated in this study if measuring TG intraoperatively is predictive for blood loss postoperatively. The CAT assay demonstrated to predict blood loss when performed intraoperatively, and especially in platelet-rich plasma.

In Chapter 6 the role of CAT and routine laboratory tests prior to CPB and after protamine administration in the prediction of blood loss postoperatively was investigated. Preoperatively, platelet count was the only routine laboratory test predictive for blood loss. Postoperatively, both platelet count and plasma fibrinogen were predictive. On the contrary, measuring thrombin generating capacity was preoperatively as well as postoperatively predictive, and this predictive value was once more higher when TG was measured in platelet-rich plasma instead of measured in platelet-poor plasma.

Another test which could reflect the coagulation capacity peroperatively is the rotational thromboelastometry method (ROTEM, TEM International, Munich, Germany). This is a viscoelastic whole blood test determining the rate of fibrin polymerization as well as the overall clot strength. It should provide information about the contribution of both fibrinogen and platelet count to clot strength. In Chapter 7 it was investigated how ROTEM and routine laboratory tests change after CPB. All variables are significantly changed from baseline directly postoperative as a consequence of CPB, but in different degrees. As a measure for the contribution of platelets, the ROTEM variable PLATEM was calculated. This variable did not show any difference compared to baseline, whereas platelet count did decrease significantly. The maximal clot strength in the FIBTEM test was strongly correlated to the plasma fibrinogen level. In addition, it was investigated whether both ROTEM as well as routine laboratory measurements were related to blood loss postoperatively. Despite of being associated with higher blood loss when outside the reference range, the predictive value of plasma fibrinogen levels and several ROTEM values were only moderately high. ROTEM demonstrated higher predictive values compared to plasma fibrinogen, however, only APTEM clot formation time en APTEM alpha preoperatively were significantly more predictive for blood loss than fibrinogen. Although ROTEM does not give a complete reflection of the coagulation cascade, it would be clinically more useful than only plasma fibrinogen because this test has shorter turnaround times.

The predictive value of ROTEM for bleeding risk postoperatively seems to be lower than the predictive value of CAT measured in plasma. Determining thrombin generation peroperatively by CAT gives a good reflection of disturbances induced by CPB 
SUMMARY

surgery. Moreover, the development of the whole blood CAT assay is on its way, and the use of a point-of-care method is likely becoming feasible in the near future. 


\section{SAMENVATTING}



Patiënten die een hartoperatie ondergaan met cardiopulmonale bypass (CPB) zijn onderhevig aan verstoringen in de hemostase. Bloedverlies is een van de meest voorkomende gevolgen, ondanks preoperatieve evaluatie middels medische anamnese en routine laboratorium stollingstesten. Andere klinische consequenties van de ontstane coagulopathie zijn rethoracotomie, postoperatieve beroerte door hypercoagulabliteit en verhoogde mortaliteit. Daarnaast bestaat een verlengde opname op de intensive care en toegenomen zorgkosten.

Verstoorde hemostase kan worden toegeschreven aan abnormale trombine generatie (TG), plaatjes dysfunctie en excessieve fibrinolyse. Hemodilutie veroorzaakt een reductie in de plasma concentratie van stollingsfactoren en bloedplaatjes tot ongeveer $50 \%$. Consumptie wordt veroorzaakt door weefselschade, contact activatie met de artificiële oppervlakten van het extracorporale circuit en de transfusie van pericard bloed. Antistolling met hoge doseringen ongefractioneerde heparine kan resulteren in verdere verstoring van de hemostase.

Primair wordt het antistollingseffect van heparine bewerkstelligd door binding met antitrombine (AT). Het meten van dit antistollingseffect is echter gecompliceerd omdat het afhankelijk is van veel variabelen die de heparine sensitiviteit van een patient bepalen, zoals: 1 ) de beschikbaarheid van AT, 2) het antistollingseffect van tissue factor pathway inhibitor (TFPI) vrijgemaakt door heparine, 3) de binding van heparine aan eiwitten in de bloedstroom, 4) de binding van heparine aan het endotheel, en 5) bloedplaatjes die zelf heparine neutraliseren maar ook middels het vrijmaken van plaatjes factor 4 (PF4) heparine neutraliseren.

Door al deze verstorende aspecten, is het controleren van de hemostatische capaciteit van de cardio chirurgische patiënt een uitdaging. Momenteel beschikken we niet over apparatuur die de hemostase en antistolling adequaat kunnen monitoren. Heparinisatie wordt bepaald middels het meten van de heparine concentratie, of door het meten van het effect van heparine middels stollingstijden. De verschillende testen om heparine concentratie te meten laten echter weinig overeenstemming zien, en het monitoren van heparine concentratie wordt ook niet uniform geassocieerd met bloedverlies. De meest gebruikelijke test gebaseerd op stollingstijd, de activated partial thromboplastin time (aPTT), is niet geschikt tijdens CPB gezien de heparine concentraties $>1 \mathrm{IE} / \mathrm{mL}$. Daarom wordt tijdens cardiochirurgie de verlenging van stolling 'pointof-care' gemeten middels de zogenaamde activated clotting time (ACT). Het eindpunt van deze volbloed test is fibrine vorming. Eigenaardig genoeg is er geen verband tussen de belangrijkste cofactor (AT) van heparine en de ACT.

In hoofdstuk 3 wordt de relatie tussen AT en de heparine sensitiviteit index (HSI) bepaald. De resultaten van deze observationele studie duiden aan dat een verlaagde heparine respons, gemeten door de ACT, niet geassocieerd is met een verlaagd AT niveau. Dit suggereert dat heparine monitoring middels ACT onbetrouwbaar is. Dit kan 
verklaard worden doordat de ACT assay slechts de initiatie fase reflecteert en niet de volledige stollingscascade. De test stopt als slechts $5 \%$ van de totale hoeveelheid trombine is gevormd, waardoor $95 \%$ van de hemostatische capaciteit onbeoordeeld blijft. Verder wordt in dit hoofdstuk aangetoond dat een hoog trombocytenaantal wel wordt geassocieerd met een verlaagde heparine respons door het meest voor de hand liggende neutraliserend effect van trombocyten. De heparine concentratie die nodig is om de stolling te remmen is direct gerelateerd aan het aantal trombocyten. Uit deze studie kan geconcludeerd worden dat het niet bereiken van een voldoende hoge ACT geen indicator is van AT deficiëntie, maar beïnvloed kan zijn door een hoog trombocytenaantal.

De ACT wordt ook beïnvloed door de technische prestatie van het type ACT apparaat. ACT apparaten gebruiken verschillende activatoren, monster volumes en detectiesystemen. Als gevolg hiervan zijn ACT waarden, gemeten met verschillende apparatuur, niet onderling vergelijkbaar. Dit wordt ook bevestigd in hoofdstuk 4, waarin de GEM PCL (Instrumentation Laboratory), de Hemochron 801 (International Technidyne Corporation) en de ACT II Automated Coagulation Timer (Medtronic) met elkaar vergeleken worden. Hoewel de reproduceerbaarheid van verschillende apparaten goed is, is er geen overeenstemming tussen de apparaten. Daarom kan geadviseerd worden om de prestatie van een nieuw ACT apparaat in ieder specifieke kliniek te evalueren in overeenstemming met de lokale antistollingsprotocollen.

$\mathrm{Er}$ is toenemend bewijs dat bloedstolling steeds verder wordt geactiveerd tijdens $\mathrm{CPB}$, ondanks het hanteren van ACT protocollen. Dit leidt tot een consumptie coagulopathie resulterend in verlengde ACT waarden, terwijl heparine niveaus dalen. Hierdoor wordt er een verkeerd beeld verkregen van de antistollingsstatus van de patiënt. Het is daarom wenselijk om een betere test te hebben die de antistollingsstatus en de volledige hemostatische capaciteit van de patiënt in kaart brengt. Routine laboratoriumtesten (zoals trombocyten aantal, fibrinogeen en D-dimeren) reflecteren niet de activiteit van zowel de stollings- als antistollingsfactoren, en de interactie met trombocyten. Daarnaast is de tijdsduur van deze testen vaak langer omdat ze in plasma gemeten worden en zijn de resultaten niet snel beschikbaar, waardoor ze niet de actuele toestand van het stollingssysteem weergeven. In hoofdstuk $\mathbf{5}$ wordt een poging gedaan om de ultieme test die alle stolling gerelateerde veranderingen voor, tijdens, en na CPB kan weergeven, aan het licht te brengen. De Calibrated Automated Thrombography (CAT) assay meet de totale hoeveelheid trombine generatie (TG) in de tijd en zou kunnen volstaan. Naast deze test worden ook de routine laboratorium testen, de ACT en TFPI gemeten. Zowel de ACT als de CAT gaven een goede weerspiegeling van de heparinisatie voorafgaande aan CPB. Om de onderliggende TG capaciteit te onderzoeken, werden gehepariniseerde samples geneutraliseerd met polybrene, wat een significante daling liet zien in de TG capaciteit direct na heparinisatie vergeleken 
met baseline. Dit zou verklaard kunnen worden door het antistollende effect van TFPI. Echter, gedurende CPB namen TG niveaus opnieuw toe. Dit procoagulant effect werd goed weerspiegeld door de CAT metingen. De ACT liet na de start van CPB een verlenging zien, dat niet overeenkomt met het procoagulant effect van CPB en de daling in AT. Tijdens CPB, correleerde de ACT ook niet meer met het niveau van heparinisatie gemeten met de anti-Xa assay. Plasma D-dimeren lieten een daling zien na de start van $\mathrm{CPB}$, welke eveneens niet het procoagulant effect van CPB weerspiegeld. Deze studie laat zien dat het mogelijk is om TG te bepalen tijdens CPB ondanks het hoge heparinisatie niveau, en dat het de hemostatische capaciteit met behulp van CAT beter wordt weergegeven dan met conventionele testen. Tevens werd in deze studie onderzocht of het meten van TG intraoperatief voorspellend is voor bloedverlies postoperatief. De CAT toonde aan het bloedverlies te kunnen voorspellen als het intraoperatief bepaald wordt en met name in plaatjes-rijk plasma.

In hoofdstuk 6 werd daarom de rol van CAT en routine laboratorium testen voorafgaande aan CPB en na protamine toediening onderzocht in de voorspelling van bloedverlies postoperatief. Preoperatief was het aantal trombocyten de enige standaard bepaling die voorspellend was voor bloedverlies. Postoperatief waren zowel het trombocytenaantal als het plasma fibrinogeen voorspellend. Het meten van de trombine generatie capaciteit middels CAT was daarentegen zowel pre- én postoperatief voorspellend en deze voorspellende waarde was wederom hoger wanneer trombine generatie werd bepaald in plaatjes-rijk plasma dan in plaatjes-arm plasma.

Een andere test die de stollingscapaciteit peroperatief zou kunnen weergeven is de tromboelastometrie methode of ROTEM (TEM International, Munich, Germany). Dit is een viscoelastische volbloed test die de mate van fibrine polymerisatie en de sterkte van het stolsel bepaalt. Het geeft informatie over de bijdrage van zowel fibrinogeen als trombocyten aan het stolsel. In hoofdstuk 7 werd onderzocht hoe ROTEM en routine laboratorium testen veranderen na CPB. Alle variabelen veranderden direct postoperatief maar in verschillende mate. Als maat voor het trombocytenaantal werd de afgeleide PLATEM gebruikt. Deze liet echter geen verschillen zien ten opzichte van baseline, terwijl het trombocytenaantal wel sterk daalde. De maximale stolselsterkte in de FIBTEM test was sterk gecorreleerd met het plasma fibrinogeen niveau. Eveneens werd gekeken of zowel de ROTEM als de routine laboratorium variabelen gerelateerd zijn aan het bloedverlies postoperatief. Ondanks het feit dat plasma fibrinogeen en verschillende ROTEM waarden buiten het referentiekader, significant geassocieerd waren met bloedverlies, was de voorspellende waarde van deze variabelen slechts matig. ROTEM liet hogere voorspellende waarden zien dan plasma fibrinogeen, echter, alleen preoperatief APTEM clot formation time en APTEM alpha waren significant meer voorspellend voor het bloedverlies dan fibrinogeen. Hoewel ROTEM niet de volledige stollingscascade in beeld brengt, zou deze test toch klinisch van meer waarde 
kunnen zijn dan alleen het plasma fibrinogeen. Dit omdat deze test in een korter tijdsbestek resultaten geeft.

ROTEM lijkt, in vergelijking met de CAT, minder discriminerend vermogen te hebben voor bloedverlies. Het bepalen van trombine generatie peroperatief middels CAT laat 'overall' een goede weerspiegeling zien van de hemostatische veranderingen bij de cardiochirugische patiënt. Daarenboven is de volbloed CAT assay in ontwikkeling, waardoor het gebruik van een volbloed point-of-care TG methode in de nabije toekomst voor de hand ligt. 


\section{VALORIZATION}



In cardiac surgical patients the hemostatic condition is preoperatively evaluated by assessment of medical history and routine coagulation tests. However, it is well known that these tests are poor predictors of either a bleeding or a thrombotic tendency. All too often patients having normal test results develop a coagulopathy leading to excessive bleeding. Studies found a mortality rate being four times higher in patients with excessive postoperative blood loss. Avoidance of postoperative hemorrhage may reduce adverse events like sepsis, acute respiratory distress syndrome, renal failure and death. These complications and the risk of re-exploration place a high demand on hospital resources like transfusion needs, ventilatory support, intensive care (ICU) support, and personnel. The associated costs directly related to these adverse events are significant. In 2014 cardio surgical patients in the $\mathrm{MUMC}^{+}$consumed $\sim 12 \%$ of the total amount of blood products ( $€ 560.000)$. Patients with a high amount of postoperative chest tube drainage have significantly more blood transfusions, but also longer hospital stays than controls. The incremental (extra) cost of excessive postoperative hemorrhage will be significant.

From a clinical perspective, it would be extremely useful to have a test that accurately predicts an individual's coagulation potential. Ideally, such a test should detect the risk for thrombosis, and diagnose, and even predict bleeding in the whole perioperative period. In addition, it should indicate the effect of medical therapy on procoagulant and anticoagulant capacity.

It was already shown in earlier studies that thromboelastography/ thromboelastometry (TEG/ROTEM) may reduce blood product consumption in coronary artery bypass grafting (CABG) patients. This method does also give a good reflection of heparin, used as anticoagulant therapy in cardiac surgery.

Literature suggests that calibrated automated thrombography (CAT) is better at identifying patients at high risk for excessive blood loss or thrombotic complications than simple clotting tests like the activated clotting time (ACT). CAT might improve the timely identification of hemostatic problems underlying a bleeding tendency perioperatively, which may improve timely and tailored transfusion management. In addition, it can be used for assessing substitution and antithrombotic treatment.

CAT is designed to measure thrombin generation in clotting plasma. However, recently CAT is developed into a method that enables the measurement of thrombin in whole blood. Also, CAT measurements including the effect of blood flow on thrombin generation are still under development. It is getting more and more an advanced test coming more closely to the physiological situation.

This thesis underlines the complexity of the disturbances in coagulation in cardiac surgical patients together with the difficulties in monitoring it. However, it can be concluded that the new, more advanced, tests ROTEM and CAT, performed pre-, per- and postoperatively, do have a predictive value for bleeding postoperatively. Measuring 
fibrinogen level is a conventional method which equals the predictive value of ROTEM and CAT for blood loss postoperatively, but it is time consuming.

There are still a lot of questions but also a lot of challenges, regarding the topic of hemostasis in cardiac surgery. The first challenge is to organize larger, clinical studies which have more impact. The sample size in our studies was small. Although it allowed us to detect significant differences in the studied parameters, the statistical power is low. In addition, our studies were single-center experiences. Transfusion thresholds, hematologic practice, discontinuation of anticoagulants, and the use of perioperative antifibrinolytics may not reflect worldwide practice, and the generalizability (validity) of our results needs thus to be proven in larger multicenter studies. In addition, intervention studies are needed. Monitoring (anti)coagulation in a correct way and predicting bleeding complications is a good fundament, but knowing what to do in this situation is the ultimate aim. When performed all these studies in a correct way, this can take another 10 years of doing research. Financial resources are required for organizing this. Health foundations should be contacted. Applications for grants are often essential: funds will considerably reducing the time needed to perform those studies, e.g. by investing in personnel and more technical resources. Gradually, we are on the right track in treating patients at risk of excessive bleeding and transfusion.

A very obvious and surprising conclusion from studies described in this thesis is that CAT and ROTEM performed preoperatively are more predictive for blood loss than performed postoperatively at admission on the ICU. Before the most impressive intervention will be done - the surgical procedure with exposure of the patient's blood to the extracorporeal circuit - it is possible to predict whether patients are at risk of bleeding. This means that a patient has already a hemostatic condition prone to hemorrhage, which can be caused by several factors, e.g. exposure to aspirin or oral anticoagulants preoperatively, renal insufficiency, hepatic disorders, sepsis or other comorbidity leading to hemostatic disturbances. Or are there causes we don't even think about? In fact, we have to deal with the consequences, instead of having to struggle with the cause of the problem as we cannot influence this most of the times. We should have a test, which is suitable for detecting all kinds of hemostatic disturbances. The results of this test should lead to an intervention which reduces the risk of bleeding for the patient without creating adverse events. This test should be available as a point-of-care test in the operating theatre, and could be performed pre- and postoperatively in the ideal situation. The challenge of developing such a test is to implement new technologies in a still more advanced monitoring system, eliminating the limitations of the current tests, reflecting the actual hemostatic condition, and improving (anti)coagulation therapy during CPB. This is not beyond our imagining, because levels of expertise and know-how have been rising constantly. Findings have to be 
translated into diagnostic tools, medicines, procedures, policies and education by using a multi-disciplinary, highly collaborative, "bench-to-bedside" approach.

More advanced self-tests, are already under development, so patients can test the coagulability of their blood at home. Continuously adjusting hemostatic therapy could be quite simple having such a self-test, as a result of which bleeding risks could be minimized preoperatively.

In addition, it would be superior to develop this method into a test which can be used also intraoperatively, e.g. in case of emergency when there is no time to care about monitoring hemostatic conditions preoperatively. A refined hemostatic assay should elucidate this during surgery. Imagine, the patient is anticoagulated and connected to the extracorporeal circuit without knowing the level of anticoagulation. Neither there is a reliable anamnesis or medical history. Especially, in this situation it is desirable to have information about the coagulation status intraoperatively.

However, a new developed method should be useful also in elective cases, simply because of the great need for such a test to classify patients with increased risk of thrombosis or bleeding. New techniques should be created considering the experience in the field of cardiopulmonary bypass we got used to and what kind of monitoring we prefer. We are familiar with continuous monitoring of blood gasses in-line in the extracorporeal circuit. That is fast and easy. Wouldn't it be innovative to monitor (anti)coagulation status in-line during cardiopulmonary bypass? Then it would approach the physiologic system rather close by measuring also flow rates. How can we develop this insight into a monitoring technique which is user-friendly: fast, cheap and easy to perform. A good collaboration between clinical perfusionists, hematologists, technicians, and companies is required to start an industrial process. Financial resources should be secured before contacting companies. Investing money is an essential thing in developing new assays. Companies are needed which have experience in the field of monitoring hemostasis and which are also close to the (cardiac surgical) clinic. They have to develop advanced hemostatic assays specific for our cardiac surgical patients. If it is possible to make a self-test, then the next step should be to convert such a small device into the extracorporeal circuit, to implement it as an in-line system for continuously monitoring intraoperatively. This can be a diagnostic tool connected to a sample line in the extra-corporeal circuit. It should be able to monitor the anticoagulation status of the patient at a specific moment, but also to give information about the underlying coagulation status when heparin would be neutralized.

Developing a new diagnostic coagulation monitoring system will be a challenge which brings a solution with a great impact on future cardiac surgical patients worldwide. 


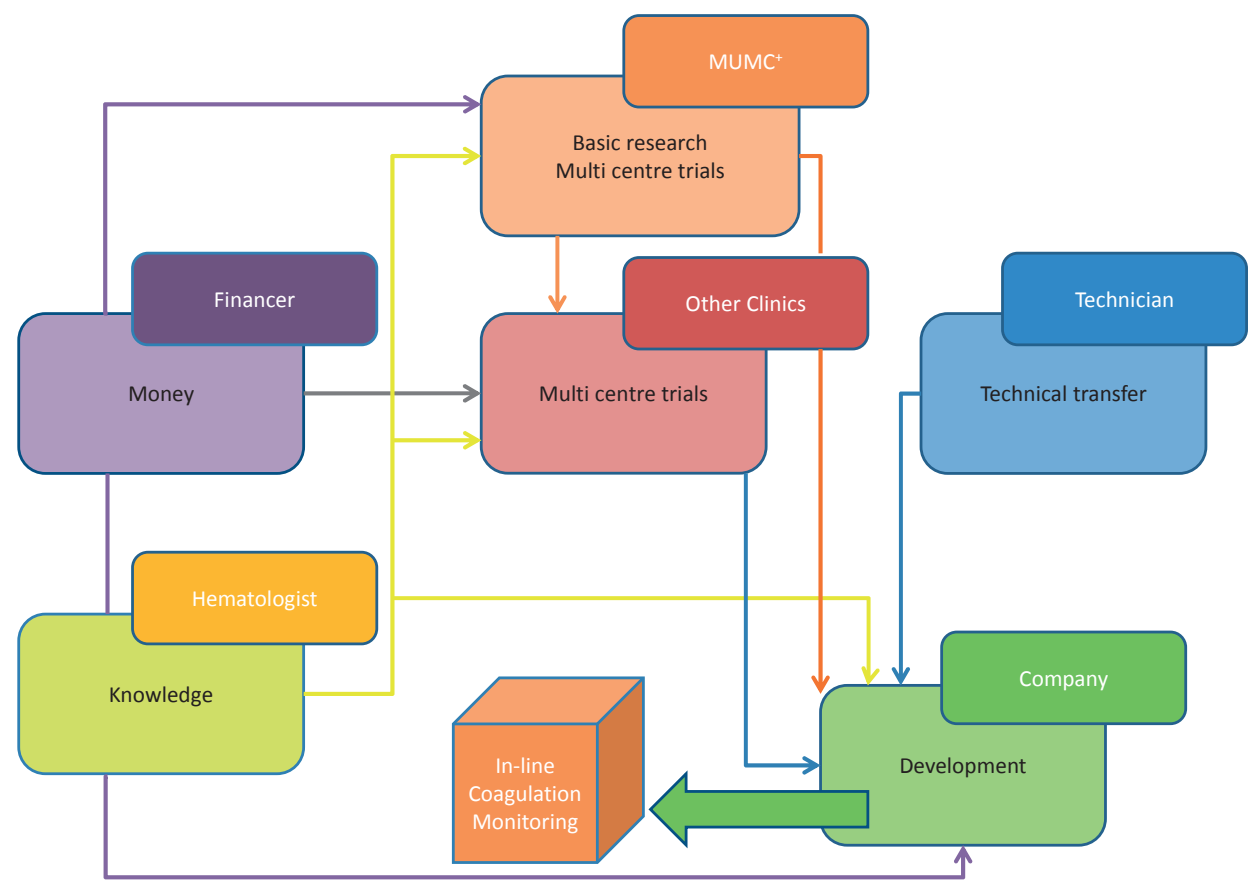

Figure 1: schematic overview of a translational approach to produce a hemostatic monitoring application that can be used point-of-care. 
DANKWOORD 

Zo... dit was het dan. De afgelopen jaren ben ik ver gekomen: Zuid-Afrika, NieuwZeeland, Tasmanië, Hawaii,... Ameland...., toch maar op safari (in de Beekse Bergen). Dat waren de ontspannende reizen. Maar de wetenschappelijk reis kostte wat meer inspanning. Bijna 10 jaar geploeterd om te pogen iets wetenschappelijks van formaat uit de printer te laten rollen.

Het dankwoord is voor mij het laatste onderdeel om te schrijven, hoewel het voor de lezer van dit boekje vaak zowel het eerste als het laatste betreft, naarstig op zoek naar zijn of haar eigen naam in het gekrioel van dankbetuigingen.

Ondanks dat mijn naam prijkt op de omslag, weet ik dat ik dit niet alleen tot stand heb gebracht. Ik ontkom er dan ook niet aan om eenieder die zijn of haar steentje bijgedragen heeft te bedanken. Sommigen zijn zeer bescheiden en willen hier niet uitvoerig bedankt worden, anderen hebben jaren hun best gedaan om juist meerdere keren genoemd te willen worden (hebben zelfs verzoeken hiervoor ingediend). Gelukkig weet iedereen zelf hoe groot zijn 'steentje' was, en wil ik hen dan ook in diezelfde mate bedanken.

Het risico ligt altijd op de loer dat er iemand vergeten wordt die zijn best heeft gedaan. Vandaar dat ik er voor kies geen personen bij naam te noemen. Ik wil dan ook het Maastricht Universitair Medisch Centrum als orgaan bedanken, welke mij de gelegenheid heeft gegeven dit onderzoek uit te voeren en af te ronden, alsmede de patiënten die bereid waren deel te nemen aan het onderzoek. Ook Synapse b.v. was een onmisbare factor.

Wetenschap en ontspanning waren gedurende de hele periode goed in balans, dus tenslotte ook een knipoog naar mijn familie, vrienden en 'sjattekes' thuis die hier aan bijgedragen hebben. 

CURRICULUM VITAE 

Yvonne Bosch is geboren op 17 januari 1979 te Roermond. In 1997 behaalde zij haar Gymnasium diploma aan de SG St. Ursula, te Horn. In hetzelfde jaar startte ze met de opleiding Gezondheidswetenschappen aan de Universiteit Maastricht en koos voor de afstudeerrichting Bewegingswetenschappen. Haar doctoraal rondde zij af in 2001, waarna ze begon met de opleiding tot Klinisch Perfusionist in het academisch ziekenhuis Maastricht. In 2004 behaalde zij haar diploma in dit vakgebied en sindsdien is zij werkzaam in hetzelfde ziekenhuis. In 2006 werd de aanzet gemaakt om verder onderzoek te gaan doen op het onderwerp van haar afstudeeronderzoek (stollingsmonitoring) mede in samenwerking met Synapse bv. Dit werd de start van haar promotietraject.

Yvonne Bosch was born in Roermond, the Netherlands, on January the 17th 1979. She graduated from scholengemeenschap St. Ursula in Horn (Gymnasium), in 1997. Immediately thereafter she started with the study Health Sciences at the University of Maastricht and chose Movement Sciences as her graduation field. She received her Master of Science degree in 2001, after which she started with her training for Clinical Perfusionist in the University Hospital Maastricht. In 2004 she received her diploma in this field and she is working in the same hospital since then. In 2006 the first onset was made do to further research on the topic of her graduation project (coagulation monitoring), together with Synapse bv. This resulted in the start of her dissertation project. 
Oktober 2015 